\title{
WestVirginiaUniversity
}

THE RESEARCH REPOSITORY @ WVU

Graduate Theses, Dissertations, and Problem Reports

2012

\section{Reversible Myocardial Dysfunction in a Rodent Model of Prenatal Stress}

Jessalyn Marie Hadfield

West Virginia University

Follow this and additional works at: https://researchrepository.wvu.edu/etd

\section{Recommended Citation}

Hadfield, Jessalyn Marie, "Reversible Myocardial Dysfunction in a Rodent Model of Prenatal Stress" (2012). Graduate Theses, Dissertations, and Problem Reports. 3512.

https://researchrepository.wvu.edu/etd/3512

This Thesis is protected by copyright and/or related rights. It has been brought to you by the The Research Repository @ WVU with permission from the rights-holder(s). You are free to use this Thesis in any way that is permitted by the copyright and related rights legislation that applies to your use. For other uses you must obtain permission from the rights-holder(s) directly, unless additional rights are indicated by a Creative Commons license in the record and/ or on the work itself. This Thesis has been accepted for inclusion in WVU Graduate Theses, Dissertations, and Problem Reports collection by an authorized administrator of The Research Repository @ WVU. For more information, please contact researchrepository@mail.wvu.edu. 


\title{
Reversible Myocardial Dysfunction in a Rodent Model of Prenatal Stress
}

Jessalyn Marie Hadfield, B.S.

Thesis submitted to the

Davis College of Agriculture, Natural Resources, and Design

West Virginia University

in partial fulfillment of the requirements

for the degree of

\section{Master of Science}

In

Reproductive Physiology

\author{
Mitchell S. Finkel, M.D., Chair \\ E.Keith Inskeep, Ph.D. \\ John M. Hollander, Ph.D. \\ Diane B. Miller, Ph.D.
}

Division of Animal and Veterinary Sciences

Morgantown, WV

2012

Keywords: Prenatal Stress, N-acetylcysteine, Heart Function 


\title{
Abstract \\ Reversible Myocardial Dysfunction in a Rodent Model of Prenatal Stress
}

\author{
Jessalyn Marie Hadfield
}

Compelling clinical reports indicate that emotional/behavioral stress alone is sufficient to cause profound, but completely reversible myocardial dysfunction in selected individuals. A rodent cardiomyopathy model was previously reported by combining pre-natal and post-natal behavioral/restraint stress (Stress). A decrease in \% FS (the ratio between the diameter of the left ventricle when it is relaxed and when it is contracted) by echocardiogram was previously reported, along with both systolic and diastolic dysfunction by catheter-based hemodynamics, as well as attenuated hemodynamic and inotropic responses to the beta adrenergic agonist, isoproterenol (ISO) in Stress compared with matched Controls. The p38mitogen-activated protein (MAP) kinase inhibitor, SB203580, both prevented and reversed the baseline reduction in systolic and diastolic dysfunction, as well as the blunted ISO response in cardiac myocytes from Stress animals in vitro and in vivo. p38 MAP kinase has been reported to be activated by oxidative stress. Previous work showed that the sulhydryl donor and substrate for glutathione, $\mathrm{N}$ acetylcysteine (NAC), reversed myocardial dysfunction in 2 different genetic proteinopathy models (Syrian Hamster and Tat mouse). In the current studies, cardiac myocytes isolated from Stress revealed evidence of mitochondrial dysfunction and oxidative stress as indicated by decreased ATP concentration and decreased GSH/GSSG ratio, respectively. Stress cardiac myocytes also displayed blunted inotropic and $\left[\mathrm{Ca}^{2+}\right]_{\mathrm{i}}$ responses to extracellular $\mathrm{Ca}^{2+}\left(\left[\mathrm{Ca}^{2+}\right]_{\text {out }}\right)$ and ISO ( $<<0.05$, for each), as well as altered inotropic responses to the intracellular calcium regulator, caffeine $(10 \mathrm{mM})$, which causes sacroplasmic reticulum $\mathrm{Ca}^{2+}$ depletion $(\mathrm{p}<0.01)$. Treatment of cardiac myocytes with NAC $\left(10^{-3} \mathrm{M}\right)$ normalized calcium handling in response to ISO and $\left[\mathrm{Ca}^{2+}\right]_{\text {out }}$ and inotropic response to caffeine ( $\mathrm{p}<0.01$, for each). NAC also normalized the blunted inotropic response to ISO and $\mathrm{Ca}^{2+}(\mathrm{p}<0.01$, for each). Surprisingly, NAC did not reverse the changes in GSH, GSSG or GSH/GSSG ratio. These data indicate that NAC may reverse cardiac myocyte dysfunction by a glutathione-independent effect on intracellular calcium signaling. Elucidating the molecular mechanisms responsible for the salutary effects of NAC may provide novel insights into potential therapeutic targets for the reversal of myocardial dysfunction in patients with cardiomyopathies and heart failure. 


\section{Table of Contents}

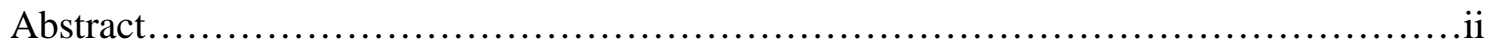

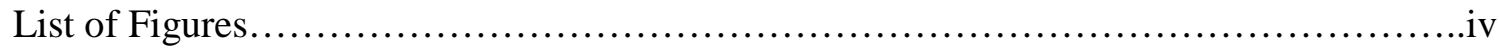

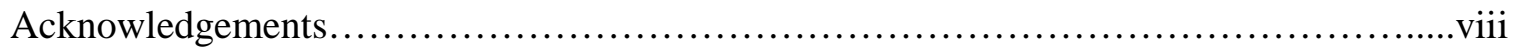

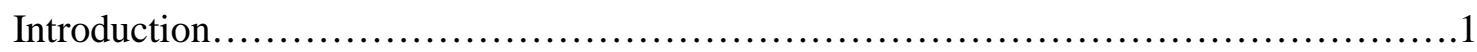

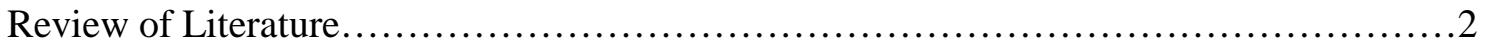

Mood Disorders and Heart Disease.................................................

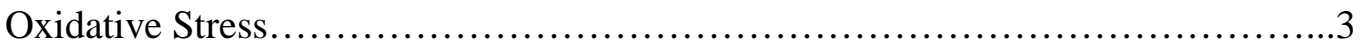

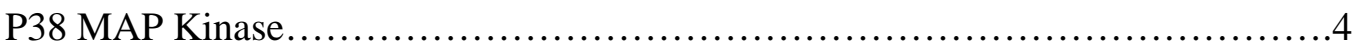

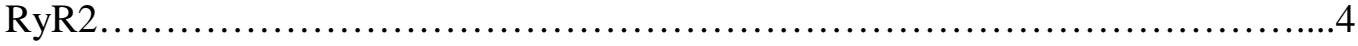

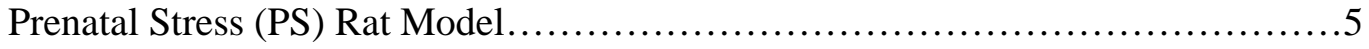

Previous Studies................................................................

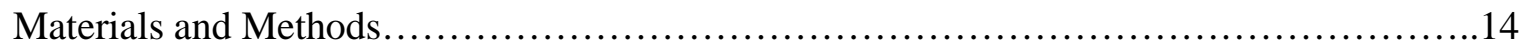

Stress Paradigm.................................................................

Isolation of adult rat ventricular myocytes......................................15

Cardiac myocyte contractile function.............................................16

Intracellular calcium measurements.........................................16

ATP concentration in heart tissue................................................

GSH/GSSG concentration in cardiac myocytes..................................17

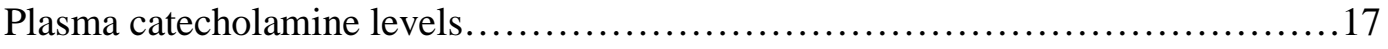

Statistical methods........................................................... 18

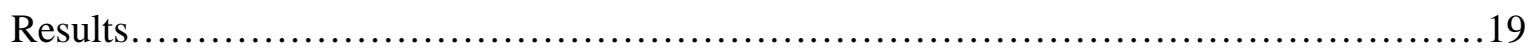

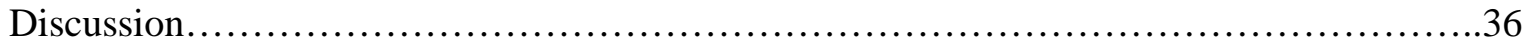

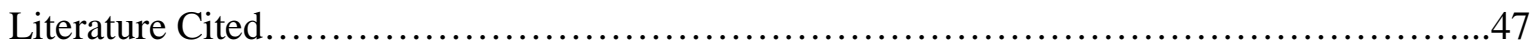




\section{List of Figures}

Figure 1: Flow diagram illustrating the steps involved in generating this prenatal stress animal model.

Figure 2: Bar graph illustrating percent Fractional shortening (\%FS) determined by echocardiogram. (61.9 \pm 2.4 vs. $45.8 \pm 3.9$, Control vs. Stress; $\mathrm{P}<0.01 ; \mathrm{n}=12)$.

Figure 3: Bar graph illustrating contractile function of adult rat ventricular myocytes reflected as

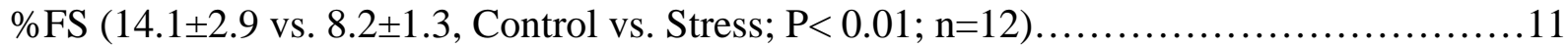

Figure 4: Bar graph illustrating the reversal of the myocardial depressant effects of Stress with the p38 MAP kinase inhibitor, SB-203580 (8.2 \pm 1.3 vs 13.3 \pm 1.7 , Stress vs. Stress + SB; $p<0.01$; $\mathrm{n}=12$ ). .11

Figure 5: Bar graphs illustrating reversibility of impaired systolic (+dp/dt, A; LVSP,B) and diastolic (-dp/dt,C) function by the p38 MAP kinase inhibitor, SB203580 $\left({ }^{*} \mathrm{p}<0.05\right.$, Stress vs. Stress + SB, $n=6-8)$.

Figure 6: Representative Western Blot (A) and densitometry analysis (B) showing increased phosphorylated p38 MAP kinase and troponin I in Stress vs. Control. The p38 MAP kinase inhibitor SB reversed the increase in phosphorylation of both p38 MAP kinase and troponin I in

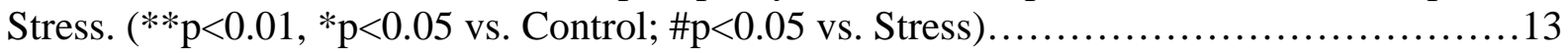

Figure 7: Bar graphs depicting plasma Norepinephrine levels ( $1.99 \pm 0.29$ vs. $11.13 \pm 0.55 \mathrm{ng} / \mathrm{ml}$, con vs. stress, ${ }^{* *} \mathrm{p}<0.01 ; \mathrm{n}=10$ ) (A); and epinephrine levels (7.60 \pm 0.80 vs. $21.01 \pm 1.02$, Con vs. Stress, $\left.{ }^{* *} \mathrm{p}<0.01 ; \mathrm{n}=10\right)(\mathrm{B})$. .19

Figure 8: Bar graph depicting ATP levels measured in extracts from homogenized whole hearts (5.16 \pm 0.36 vs. $2.42 \pm 0.16 \mu \mathrm{M} / \mathrm{g}$ total protein, Con vs. Stress, ${ }^{* *} \mathrm{p}<0.05 ; \mathrm{n}=10$ ). 20

Figure 9: Bar graph depicting GSH levels (29.24 $\pm 3.84 v s$ 23.63 $\pm 2.85 \mu \mathrm{M} / \mathrm{g}$, Con vs. Stress, $\mathrm{p}=\mathrm{NA} ; \mathrm{n}=10)(\mathrm{A})$; GSSG levels $\left(1.23 \pm 0.27 \mathrm{vs} 5.46 \pm 0.69 \mu \mathrm{M} / \mathrm{g}\right.$, Con vs. Stress, $\left.{ }^{* *} \mathrm{p}<0.01 ; \mathrm{n}=10\right)$ (B); and GSH/GSSG ratio (23.76 \pm 4.20 vs 5.58 \pm 1.42 , Con vs. Stress, $\left.{ }^{* *} \mathrm{p}<0.01 ; \mathrm{n}=10\right)(\mathrm{C})$ measured from isolated cardiac myocytes.

Figure 10: Baseline percent peak shortening (\%PS) in control and stress myocytes with and without the addition of NAC $\left(10^{-3} \mathrm{M}\right)\left(11.78 \pm 0.34\right.$ vs. $7.09 \pm 0.38 \%$, Con vs. Stress, ${ }^{* *} \mathrm{p}<0.01$; $7.09 \pm 0.38$ vs. $12.34 \pm 0.59 \%$, Stress vs. Stress + NAC, $\# \# p<0.01 ; n=15)$. .21

Figure 11: Graphic depiction of the response of cardiac myocytes to increasing concentrations of the beta-adrenergic agonist, isoproterenol, alone and following incubation with NAC $\left(10^{-3} \mathrm{M}\right)$ on percent peak shortening (\%PS) (Con vs. Stress, $\mathrm{p}<0.01$; Stress vs. Stress + NAC, $\mathrm{p}<0.01$; $\mathrm{n}=15$ ) (A), positive inotropy $(+\mathrm{dl} / \mathrm{dt}$ ) (Con vs. Stress, $\mathrm{p}<0.01$; Stress vs. Stress $+\mathrm{NAC}, \mathrm{p}<0.01$; $\mathrm{n}=15$ ) (B), and negative inotropy (-dl/dt) (Con vs. Stress, $\mathrm{p}<0.01$; Stress vs. Stress + NAC,

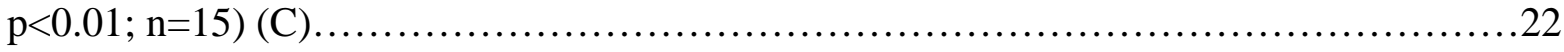


Figure 12: Graphic depiction of the response of cardiac myocytes to increasing concentrations of the beta-adrenergic agonist, isoproterenol, alone and with incubation with NAC $\left(10^{-3} \mathrm{M}\right)$ on: change in $\mathrm{Ca}^{2+}$ concentration (Con vs. Stress, $\mathrm{p}<0.01$; Stress vs. Stress + NAC, $\mathrm{p}<0.01 ; \mathrm{n}=15$ ) (A), $\mathrm{Ca}^{2+}$ departure velocity (Con vs. Stress, $\mathrm{p}<0.01$; Stress vs. Stress $\left.+\mathrm{NAC}, \mathrm{p}<0.01 ; \mathrm{n}=15\right)(\mathrm{B})$, and $\mathrm{Ca}^{2+}$ return velocity (Con vs. Stress, $\mathrm{p}<0.01$; Stress vs. Stress $\left.+\mathrm{NAC}, \mathrm{p}<0.01 ; \mathrm{n}=15\right)(\mathrm{C}) . .23$

Figure 13: Graphic depiction of the response of cardiac myocytes to increasing concentrations of extracellular $\mathrm{Ca}^{2+}$ alone and following incubation with NAC $\left(10^{-3} \mathrm{M}\right)$ on percent peak shortening (\%PS) (Con vs. Stress, $\mathrm{p}<0.01$; Stress vs. Stress + NAC, $\mathrm{p}<0.01 ; \mathrm{n}=15)(\mathrm{A})$, positive inotropy $(+\mathrm{dl} / \mathrm{dt})($ Con vs. Stress, $\mathrm{p}<0.01$; Stress vs. Stress $+\mathrm{NAC}, \mathrm{p}<0.01 ; \mathrm{n}=15)(\mathrm{B})$, and negative inotropy (-dl/dt) (Con vs. Stress, $\mathrm{p}<0.01$; Stress vs. Stress + NAC, $\mathrm{p}<0.01 ; \mathrm{n}=15)(\mathrm{C}) \ldots . . . .24$

Figure 14: Graphic depiction of the response of cardiac myocytes to increasing concentrations of extracellular $\mathrm{Ca}^{2+}$ alone and following incubation with NAC $\left(10^{-3}\right)$ on : change in $\mathrm{Ca}^{2+}$ concentration (Con vs. Stress, $\mathrm{p}<0.01$; Stress vs. Stress + NAC, $\mathrm{p}<0.01 ; \mathrm{n}=15$ ) (A), $\mathrm{Ca}^{2+}$ departure velocity (Con vs. Stress, $\mathrm{p}<0.01$; Stress vs. Stress $+\mathrm{NAC}, \mathrm{p}<0.01 ; \mathrm{n}=15$ ) (B), and Ca ${ }^{2+}$ return velocity (Con vs. Stress, $\mathrm{p}<0.01$; Stress vs. Stress + NAC, $p<0.01 ; n=15)(C) . \ldots . . . .25$

Figure 15: Tracing of control and stress cardiac myocyte contraction preceding and following exposure to caffeine $(10 \mathrm{mM})(\mathrm{A})$ and the tracing of intracellular $\mathrm{Ca}^{2+}$ concentration $(\mathrm{B})$......26

Figure16. Bar graph depicting of \% PS of control and stress cardiac myocytes before and after exposure to caffeine $(10 \mathrm{mM})\left(10.10 \pm 1.27\right.$ vs. $6.68 \pm 0.77$, Con vs. Stress, ${ }^{* *} \mathrm{p}<0.01 ; 10.10 \pm 1.27$ vs. $5.8 \pm 0.66$, Con vs. Con+Caff, $* * \mathrm{p}<0.01 ; 6.68 \pm 0.77$ vs. $1.88 \pm 0.93$, Stress vs. Stress+Caff, \#\# $\mathrm{p}<0.01 ; \mathrm{n}=15)(\mathrm{A})$; and percentage change of control and stress cardiac myocytes after exposure to caffeine $(10 \mathrm{mM})\left(44.99 \pm 5.75\right.$ vs $70.60 \pm 5.09 \%$, Con vs. Stress, $\left.{ }^{* *} \mathrm{p}<0.01 ; \mathrm{n}=15\right)(B)$.

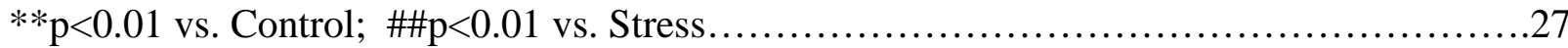

Figure 17: Bar graph depicting positive inotropy $(+\mathrm{dl} / \mathrm{dt})$ of control and stress cardiac myocytes before and after exposure to caffeine $(10 \mathrm{mM})\left(206.98 \pm 10.41\right.$ vs. $122.74 \pm 11.24$, Con vs. Stress, ${ }^{* *}$ $\mathrm{p}<0.01 ; 206.98 \pm 10.41$ vs. $164.10 \pm 11.08$, Con vs. Con+Caff, $* * \mathrm{p}<0.01 ; 122.74 \pm 11.24$ vs. 67.98 \pm 16.04 , Stress vs. Stress+Caff, \#\# $\mathrm{p}<0.01 ; \mathrm{n}=15)(\mathrm{A})$; and percentage change of control and stress cardiac myocytes after exposure to caffeine $(10 \mathrm{mM})(21.59 \pm 3.77$ vs $40.96 \pm 3.82 \%$, Con vs. Stress, $\left.{ }^{* *} \mathrm{p}<0.01 ; \mathrm{n}=15\right)(\mathrm{B}) .{ }^{* *} \mathrm{p}<0.01$ vs. Control; $\# \# \mathrm{p}<0.01$ vs. Stress..................27

Figure 18: Bar graph depicting negative inotropy (-dl/dt) of control and stress cardiac myocytes before and after exposure to caffeine $(10 \mathrm{mM})\left(-126.56 \pm 8.06\right.$ vs. $-53.28 \pm 6.84$, Con vs. Stress, ${ }^{* *}$ $\mathrm{p}<0.01 ;-126.56 \pm 8.06$ vs. $-104.23 \pm 9.32$, Con vs. Con+Caff, $* * \mathrm{p}<0.01 ;-53.28 \pm 6.84$ vs. 21.53 \pm 8.24 , Stress vs. Stress+Caff, \#\# $\mathrm{p}<0.01 ; \mathrm{n}=15$ ) (A); and percentage change of control and stress cardiac myocytes after exposure to caffeine $(10 \mathrm{mM})(17.78 \pm 2.17$ vs. $59.41 \pm 3.46 \%$, Con vs. Stress, $\left.{ }^{* *} \mathrm{p}<0.01 ; \mathrm{n}=15\right)(\mathrm{B}) .{ }^{* *} \mathrm{p}<0.01$ vs. control $\quad \# \# \mathrm{p}<0.01$ vs. stress..................28 
Figure 19: Bar graph depicting change in calcium concentration in control and stress cardiac myocytes before and after exposure to caffeine $(10 \mathrm{mM})(0.75 \pm 0.12 \mathrm{vs}$. $0.53 \pm 0.07$, Con vs. Stress, ${ }^{* *} \mathrm{p}<0.01 ; 0.75 \pm 0.12$ vs. $0.57 \pm 0.07$, Con vs. Con+Caff, $* \mathrm{p}<0.05 ; 0.53 \pm 0.07$ vs. $0.20 \pm 0.06$, Stress vs. Stress + Caff, $\# \# p<0.01 ; n=15$ ) (A); and percentage change of control and stress cardiac myocytes after exposure to caffeine $(10 \mathrm{mM})(37.72 \pm 5.68$ vs $60.02 \pm 1.04 \%$, Con vs. Stress, $\left.{ }^{* *} \mathrm{p}<0.01 ; \mathrm{n}=15\right)(\mathrm{B}) .{ }^{*} \mathrm{p}<0.05$ vs. control; ${ }^{* *} \mathrm{p}<0.01$ vs. control; $\# \# \mathrm{p}<0.01$ vs. stress

Figure 20: Bar graph depicting calcium departure velocity in control and stress cardiac myocytes before and after exposure to caffeine $(10 \mathrm{mM})(123.60 \pm 6.70$ vs. $52.90 \pm 5.64$, Con vs. Stress, $* *$ $\mathrm{p}<0.01 ; 123.60 \pm 6.70$ vs. $80.54 \pm 8.91$, Con vs. Con+Caff, $* * \mathrm{p}<0.01 ; 52.90 \pm 5.64$ vs. 24.19 \pm 7.34 , Stress vs. Stress+Caff, \#\# $\mathrm{p}<0.01 ; \mathrm{n}=15)(\mathrm{A})$; and percentage change of control and stress cardiac myocytes after exposure to caffeine $(10 \mathrm{mM})(33.65 \pm 6.97$ vs $56.74 \pm 5.11 \%$, Con vs. Stress, ** $\mathrm{p}<0.01 ; \mathrm{n}=15)(B) .{ }^{* *} \mathrm{p}<0.01$ vs. Control; \#\#p<0.01 vs. Stress.

Figure 21: Bar graph depicting calcium return velocity in control and stress cardiac myocytes before and after exposure to caffeine $(10 \mathrm{mM})\left(-99.70 \pm 6.88\right.$ vs. $-49.07 \pm 8.07$, Con vs. Stress, ${ }^{* *}$ $\mathrm{p}<0.01 ;-99.70 \pm 6.88$ vs. $-71.94 \pm 8.80$, Con vs. Con+Caff, $* * \mathrm{p}<0.01 ;-49.07 \pm 8.07$ vs. 25.69 \pm 4.52 , Stress vs. Stress + Caff, \#\# $\mathrm{p}<0.01$; $\mathrm{n}=15)(\mathrm{A})$; and percentage change of control and stress cardiac myocytes after exposure to caffeine $(10 \mathrm{mM})(23.21 \pm 4.42 \mathrm{vs} 50.88 \pm 6.92 \%$, Con vs. Stress, $\left.{ }^{* *} \mathrm{p}<0.01 ; \mathrm{n}=15\right)(\mathrm{B}) .{ }^{* *} \mathrm{p}<0.01$ vs. Control; \#\#p<0.01 vs. Stress....................29

Figure 22: Graphic depiction comparing recovery of stress versus control cardiac myocytes after exposure to caffeine $(10 \mathrm{mM})$. Results are measured as baseline, caffeine exposure and time since caffeine exposure: percent peak shortening (\%PS) ( $<<0.01$ control vs. stress) (A); positive inotropy ( $+\mathrm{dl} / \mathrm{dt})(\mathrm{p}<0.01$ control vs. stress) (B); and negative inotrophy $(-\mathrm{dl} / \mathrm{dt})(\mathrm{p}<0.01$ control vs. stress) (C); $n=15$ myocytes. 30

Figure 23: Graphic depiction comparing recovery of stress versus control cardiac myocytes after exposure to caffeine $(10 \mathrm{mM})$. Results are measured as baseline, caffeine exposure and time since caffeine exposure: change in $\mathrm{Ca}^{2+}$ concentration $\left(\mathrm{p}<0.05\right.$ control vs. stress) (A); $\mathrm{Ca}^{2+}$ departure velocity ( $\mathrm{p}<0.01$ control vs. stress) (B); and $\mathrm{Ca}^{2+}$ return velocity ( $\mathrm{p}<0.01$ control vs. stress) (C); $\mathrm{n}=15$ myocytes. ...31

Figure 24: Tracing of control and stress cardiac myocyte contraction (A) and intracellular $\mathrm{Ca}^{2+}$ concentration (B) following 30 minutes of perfusion of KHB alone or with NAC $\left(10^{-3} \mathrm{M}\right)$ after exposure to caffeine $(10 \mathrm{mM})$. 32

Figure 25: Dose response curve of NAC and its effect on cardiac myocyte recovery after exposure to caffeine (10mM). Results are measured as baseline, caffeine exposure, and $30 \mathrm{~min}$. after caffeine exposure with perfusion in buffer alone or NAC; percent peak shortening (\%PS) $(\mathrm{p}<0.01$, control vs. stress) (A); positive inotrophy $(+\mathrm{dl} / \mathrm{dt})(\mathrm{p}<0.01$ control vs. stress)(B); negative inotrophy $(-\mathrm{dl} / \mathrm{dt})(\mathrm{p}<0.01$ control vs. stress $)(\mathrm{C}) ; \mathrm{n}=15$ myocytes for each concentration

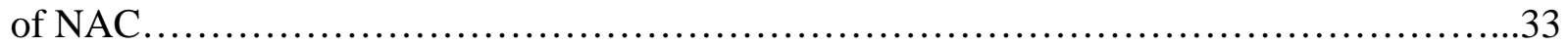


Figure 26: Dose response curve of NAC and its effect on cardiac myocyte recovery after exposure to caffeine $(10 \mathrm{mM})$. Results are measured as baseline, caffeine exposure, and $30 \mathrm{~min}$. after caffeine exposure with perifusion in buffer alone or NAC. Different myocytes were used for each concentration of NAC; change in $\mathrm{Ca}^{2+}$ concentration ( $\mathrm{p}<0.01$, control vs. stress) (A); $\mathrm{Ca}^{2+}$ departure velocity ( $\mathrm{p}<0.01$ control vs. stress) $(\mathrm{B})$; $\mathrm{Ca}^{2+}$ return velocity ( $\mathrm{p}<0.01$ control vs. stress)(C); $\mathrm{n}=15$ myocytes for each concentration of NAC .................................34

Figure 27: Bar graph depicting the effects of $10^{-3}$ NAC on GSH concentration $(29.24 \pm 3.84$ vs. $23.63 \pm 2.85$, control vs. stress; $23.63 \pm 2.85$ vs. $29.88 \pm 3.50$ Stress vs. stress $+\mathrm{NAC} p=N S \mathrm{n}=15$ ) (A), GSSG concentration ( $1.23 \pm 0.27$ vs. $5.46 \pm 0.69$, control vs. stress ** $\mathrm{p}<0.01 ; 1.86 \pm 0.26$ vs. $6.20 \pm 0.60$ control + NAC vs. stress $+\mathrm{NAC},{ }^{* *} \mathrm{p}<0.01 ; 5.46 \pm 0.69$ vs. $6.20 \pm 0.60$, stress vs. stress + NAC, $\mathrm{p}=\mathrm{NS} ; \mathrm{n}=15)(\mathrm{B})$, and on the GSH/GSSG ratio (23.76 \pm 4.20 vs. $5.58 \pm 1.42$, Control vs. Stress, ${ }^{* *} \mathrm{p}<0.01 ; 5.58 \pm 1.42$ vs. $4.66 \pm 0.52$ Stress vs. Stress $+\mathrm{NAC}, \mathrm{p}=\mathrm{NS} ; 17.93 \pm 2.30$ vs.

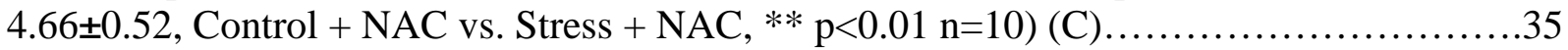

Figure 28: Schematic diagram illustrating the movement of $\mathrm{Ca}^{2+}$ into the myocyte to cause intracellular $\mathrm{Ca}^{2+}$ release followed by resequestration of $\mathrm{Ca}^{2+}$ back into the sarcoplasmic reticulum (SR) and extrusion back into the extracellular space. A small amount of $\mathrm{Ca}^{2+}$ enters the myocyte through L-type calcium channels (LTCC) which triggers a large release of $\mathrm{Ca}^{2+}$ from the SR through activation of the Ryanodine receptor (RyR2). $\mathrm{Ca}^{2+}$ is resequestered into the SR by the sacro-endoplasmic reticulum calcium ATPase $\left(\mathrm{Ca}^{2+} /\right.$ ATPase; SERCA2). $\mathrm{Ca}^{2+}$ is extruded from the cell largely through the sodium/calcium exchanger (NCX) and the plasmalemmal calcium ATPase. Contraction can be modulated through the G-protein coupled beta adrenergic receptor. Binding to the beta-receptor will lead to adenylyl cyclase activation, cyclic AMP production and PKA activation. The targets of PKA include the LTCC, RYR2, and phospholamban (regulatory protein of SERCA2). Therefore, beta-receptor activation typically leads to enhanced contraction and relaxation of the myocyte. (Modified from Kan and Finkel, 2003) 


\section{Acknowledgements}

I would like to thank all of my committee members for their contributions and insights into this project. Thanks to my major advisor, Dr. Finkel, who provided me with the guidance and means to carry out this research. This would not have been possible without him. I would like to thank Dr. Inskeep for his input on the project as well as his assistance with other aspects of my academic career. Thanks to Dr. Hollander for his advice on oxidative stress, and Dr. Miller for designing and coordinating the catecholamine assays. Also, a special thanks to Fangping Chen, $\mathrm{PhD}$, without whom, the myocyte data could not have been collected. I would also like to thank my fellow graduate students for their friendship during the last two years. Finally, I would like to thank my family. Particularly my parents and husband; I could not have completed this or my previous degree without your support. 


\section{Introduction}

A considerable body of literature supports an important relationship between chronic mood disorders (e.g. depression, anxiety) and adverse outcomes from heart disease. These results also indicate that depression mediates adverse effects on patients through mechanisms other than poor compliance to medical therapy or increases in risk factors, such as smoking. An intriguing possibility is that mood disorders and heart disease interact through a more fundamental, biological mechanism. In an effort to understand the molecular mechanisms involved, our lab developed an animal model for co-morbid mood disorders and heart disease using Birkle’s prenatal stress (PS) rat model (Cratty et al,1995).

PS has gained widespread acceptance as an animal model for chronic behavioral stress and mood disorders in humans. PS rats share important characteristic behavioral, anatomical and biochemical features with humans suffering from post-traumatic stress disorder (PTSD) and major depressive disorder (MDD) (Fride and Weinstock, 1988; Weinstock and Fride, 1988; Takahashi et al,1988; Takahashi et al, 1990; Fride et al, 1986; Weinstock et al, 1998; Cratty et al,1995; Weinstock 1997; Coplan et al, 1995). PS rats from Birkle’s lab revealed no significant echocardiographic or hemodynamic changes from control rats at 6 weeks of age. However, further behavioral stress alone in PS resulted in both systolic and diastolic myocardial dysfunction and beta adrenergic desensitization in vivo and cardiac myocyte dysfunction in vitro. Inhibition of p38 mitogen activated protein kinase (MAPK) phosphorylation both prevented and reversed the myocardial dysfunction and adrenergic desensitization in vivo and in vitro. Being a stress-activated protein kinase, p38 MAPK is activated by oxidative stress. This result indicates a pathogenic role for enhanced oxidative stress in the myocardial dysfunction in these behaviorally stressed animals. Finkel et al (1993) previously reported indirect evidence for reversal of myocardial dysfunction by redox mediated regulation of the cardiac sarcoplasmic reticulum calcium release channel, ryanodine receptor (RyR2). Therefore, we now studied the molecular mechanisms linking oxidative stress and RyR2 to reversible myocardial dysfunction following behavioral stress. 


\section{Review of Literature}

\section{Mood Disorders and Heart Disease:}

A substantial body of literature supports an important relationship between chronic mood disorders (e.g. depression, anxiety) and adverse outcomes from heart disease (Black, 1998; Sutor et al, 1998; Lauzon et al, 2003; Pignay-Demaria et al, 2003). Depressed individuals are more likely to develop heart disease in the first place, and they are more likely to die once they are diagnosed with it (Anda et al, 1993; Barefoot et al, 1996; Frasure-Smith, 1993; Frasure-Smith, 1995). Epidemiologic studies initially appeared to provide lifestyle explanations for the adverse impact of depression on the development and progression of heart disease (Glassman et al, 1990; Ziegelstein et al,2000; Blumenthal et al,1982; Carney et al, 1995; DiMatteo et al, 2002; Guck et al, 2003; Carney et al, 1993; Khanna et al, 2006). However, multivariate analyses indicate that co-morbid depression is an independent risk factor for increased cardiac death in patients with heart disease (Frasure-Smith et al, 1993; Frasure-Smith et al, 1995; Lesperance et al, 2002; Glassman et al, 2002). Clinical data also indicate that depression mediates adverse effects on patients through mechanisms other than poor compliance to medical therapy or increases in risk factors, such as smoking (Lesperance et al, 2002; Glassman et al, 2002). An intriguing possibility is that mood disorders and heart disease interact through a more fundamental, biological mechanism.

Our lab collaborated with Dale Birkle, $\mathrm{PhD}$ to develop an animal model for heart disease and depression, using her prenatally stressed rat model (PS) (Cratty et al, 1995). This model exhibited myocardial dysfunction following behavioral stress alone, without any of the other known mechanical, chemical or infectious insults. Reports supporting the clinical relevance of an association between acute behavioral stress alone and myocardial dysfunction appeared in 
studies of "stress-induced”, “Takotsubo” or "Broken Heart” cardiomyopathy (Akashi et al, 2008; Kurisu et al, 2002; Wittstein et al, 2005; Sharkey et al, 2005). Characteristics of this pathology include close temporal association with behavioral stress, complete reversibility within weeks and elevated circulating catecholamines. Although "Takotsubo" cardiomyopathy is mainly described in post-menopausal women, it is likely that more cases of stress-induced cardiomyopathy and reversible myocardial dysfunction will emerge clinically (Khanna et al, 2006; Kan and Finkel, 2003; Bybee and Prasad et al, 2008; Richard, 2011).

\section{Oxidative Stress:}

Oxidative stress is one potential biologic mechanism relating behavioral stress to adverse cardiovascular outcomes (Jones, 2008; Akki et al, 2009; Masood et al, 2008; Bouayed et al, 2007; Liu et al, 1996; Liu et al, 2003; Nadeem et al, 2006; Ahlbom et al, 2000; Kamper et al, 2009; Zhu et al, 2004; Song et al, 2009). A convincing volume of literature supports a role for oxidative stress in both behavioral stress and heart disease (Jones, 2008; Akki et al, 2009; Masood et al, 2008; Bouayed et al, 2007; Liu et al, 1996; Liu et al, 2003; Nadeem et al, 2006; Ahlbom et al, 2000; Kamper et al, 2009; Zhu et al, 2004; Song et al, 2009). Oxidative stress results from an imbalance between the production of reactive oxygen species (ROS) and the anti-oxidant capacity of the cell. The most widely accepted principle source of ROS is the mitochondrial electron transport chain. Electrons are transferred in a series of thermodynamically favorable steps to form $\mathrm{H}_{2} \mathrm{O}$ and ATP from $\mathrm{O}_{2}$. This is a highly efficient system but still leads to the production of oxygen with highly reactive unpaired electrons (e.g. superoxide, hydroxyl radical) and hydrogen peroxide. In addition, oxygen can interact with nitric oxide (NO) to form peroxynitrite and other reactive nitrogen species. Other potential sources of ROS include NADPH oxidase, cytochrome P450 and xanthine oxidase. Potent 
antioxidant enzyme systems have evolved to prevent oxidative damage. These include superoxide dismutase, catalase and glutathione peroxidase. A delicate balance exists between the production of intracellular oxidants and anti-oxidants to maintain cellular homeostasis. Enhanced oxidation and/or inadequate reducing capacity results in uncontrolled free radicals and oxidative stress (Zafarullah et al, 2003; Atkuri et al, 2007).

\section{P38 MAP Kinase:}

An important relationship between p38 MAP kinase in the brain and behavioral stress has been reported (Bruchas et al, 2007). A major characteristic of depression, dysphoria, was produced in a murine model by activation of p38 MAP kinase by opioid receptors. These and other animal studies provide the idea that enhanced oxidative stress and p38 MAP kinase activation can cause mood disorders, and are not simply a consequence of disordered mood. This concept indicates that patients who suffer from both depression and heart disease may share a common underlying biological defect that results in enhanced oxidative stress and p38 MAP kinase activation in both the CNS and myocardium (Jones, 2008; Masood et al, 2008; Bruchas et al, 2007; Li et al, 2009).

\section{RyR2:}

Possible molecular mechanisms linking oxidative stress to myocardial dysfunction include redox-mediated changes in myocardial excitation contraction coupling (E-C Coupling). The cardiac myocyte sarcoplasmic reticulum (SR) calcium release channel ryanodine receptor (SRCRC2; RyR2) plays a central role in this process (Kushnir and Marks, 2010; Prosser et al, 2010; Shan et al, 2010a; Shan et al, 2010b; Gonzalez et al, 2010; Tang et al, 2010). E-C coupling begins with electrical depolarization of the cardiac myocte that allows a minute quantity of extracellular calcium to traverse the sarcolemmal L-type calcium channel (LTCC) to activate the 
RyR2 to release a much larger quantity of SR calcium into the cytoplasm. The cytoplasmic $\mathrm{Ca}^{2+}$ then binds to Troponin $\mathrm{C}$ enabling actin-myosin binding and sliding of the myofilaments resulting in sarcomere shortening and myocardial contraction. The calcium is sequestered back into the SR through the energy-dependent sarco-endoplasmic reticulum calcium ATPase (SERCA-2) (Kushnir and Marks, 2010; Prosser et al, 2010). Thus, a direct relationship exists between the capacity of the SR to release and sequester calcium and the force of myocardial systolic contraction and diastolic relaxation. Dr. Guy Salama at UPMC was the first to describe redox regulation of the RyR2 through reactive disulfides (Prabhu and Salama, 1990). A defect in sulfhydryl gating of the RyR2 in a genetic hamster cardiomyopathy model using the sulfhydryl donor, N-acetylcysteine (NAC) was then reported (Finkel et al, 1993). A defect in dystrophin was subsequently shown by others to be responsible for the genetic hamster cardiomyopathy (Sakamoto et al, 1997). NAC has also recently been shown to be cardioprotective in the mdx dystrophin mouse model of muscular dystrophy (Williams et al, 2007). The mechanism of the benefit of NAC was not explained. However, NAC did not reduce free radical generation by NADPH (Williams et al, 2007). An alternative possibility is an effect of NAC on sulhydryl regulation of RyR2. This possibility has been suggested by recent reports of the pathogenic role of phosphorylation, nitrosylation, and oxidation of RyR2 in cardiomyopathies and heart failure (Kushnir and Marks, 2010; Prosser et al, 2010).

\section{Prenatal Stress (PS) Rat Model:}

Prenatal stress is defined as "stress experienced by the pregnant mother which affects the development of the offspring” (Braastad, 1998). In late pregnancy, the hypothalamic-pituitaryadrenal (HPA) axis is hyporesponsive to stressors and the fetus is protected from maternal glucocorticoids by placental $11 \beta$-hydroxysteroid dehydrogenase type 2 (inactivates 
corticosterone) (Mastorci et al, 2009). However, with repeated exposure to stress these defensive mechanisms fail, resulting in programming of the offspring's HPA axis due to transplacental crossing of glucocorticoids (Burnton, 2010; Maccari \& Fletcher, 2007; Barbazanges et al, 1996). The prenatally stressed rat has gained acceptance as an animal model for mood disorders because it shares important characteristic behavioral, anatomical and biochemical features with patients suffering from post-traumatic stress disorder (PTSD) and major depressive disorder (MDD) (Fride et al, 1988; Weinstock et al, 1988; Takahashi et al, 1988; Takahashi et al, 1990; Fride et al, 1986; Weinstock et al, 1998; Cratty et al, 1995; Weinstock, 1997; Coplan et al, 1996). Increased fearfulness and avoidance behaviors; anatomical alterations in the amygdala and biochemical changes in the HPA axis are shared by this animal model and patients with PTSD and MDD (Fride et al, 1988; Weinstock et al, 1988; Takahashi et al, 1988; Takahashi et al, 1990; Fride et al, 1986; Weinstock et al, 1998; Cratty et al, 1995; Weinstock, 1997; Coplan et al, 1996).

Offspring of dams that are subjected to stressful stimuli in their third trimester of pregnancy (PS) show behavioral disturbances indicative of increased fearfulness (Fride et al, 1988; Weinstock et al, 1988; Takahashi et al, 1988; Takahashi et al, 1990; Fride et al, 1986; Weinstock et al, 1998; Cratty et al, 1995; Weinstock, 1997; Coplan et al, 1996). Compared to control rats, adult prenatally stressed offspring display increased anxiety-related behaviors, including decreased locomotion and exploration and increased freezing when exposed to novelty (Wakshlak et al, 1990; Vallée et al,1997; Tazumi et al, 2005), suppressed open arm exploration in the elevated plus maze (Zimmerberg and Blaskey,1998; Vallée et al,1997; Brunton \& Russell,2010), increased defensive withdrawal (Ward et al, 2000), a decreased tendency to play (Takahashi et al,1992), and altered social interactions (Schneider, 1992; 
Clarke \& Schneider, 1993). Various modifications of the PS rat paradigm have been developed using different specific stressors including immobilization (Deminiére et al,1992; Henry et al,1994; Ward \& Weisz,1984; Frye \& Orecki, 2002), noise (Fride \& Weinstock,1984), electric shocks (Takahashi \& Kalin,1991; Estanislau \& Morato, 2005), hypoxia (Fan et al, 2009), immune challenge (Nilsson et al,2001), temperature changes (Tazumi et al, 2005), saline injection (Ward et al, 2000), or a combination of stressors (Koenig et al,2005; Richardson et al,2006). A common feature of adult PS offspring is enhanced sympatho-adrenal activation in response to stressful stimuli (Fride et al, 1988; Weinstock et al, 1988; Takahashi et al, 1988; Takahashi et al, 1990; Fride et al, 1986; Weinstock et al, 1998; Cratty et al, 1995; Weinstock, 1997; Coplan et al, 1996). These stressors cause a rise in corticotrophins and glucocorticoids in the dam (Takahashi et al, 1998; Williams et al, 1999). The fetal HPS axis responds to maternal stress during late gestation (Ohkawa et al, 1991; Williams et al, 1999), with the fetal adrenal glands able to secrete corticosterone from embryonic day 16 (Ohkawa et al,1991; Williams et al, 1999). The altered behavioral responses of PS offspring to novelty are likely due to increased or prolonged activity of the HPA axis due to impaired negative feedback of glucocorticoids (Braastad, 1998; Maccari and Fletcher, 2007; Mastorci et al, 2009)

Birkle developed one such model to study the effects of behavioral stress on the developing, juvenile and adult brain using PS rats (Cratty et al, 1995; Ward et al, 2000; Salm et al, 2004). Her data have shown that stressing pregnant rats with handling, daily saline injection and exposure to a novel environment during the last week of gestation (G14-G21) produced offspring (PS) that are more susceptible to the behavioral and neuroendocrine effects of stress as adults. These offspring have enlarged adrenal glands, elevated 
corticotropin releasing factor (CRF), elevated circulating corticosterone levels, increased heart rates, and enlarged brain amygdalae (involved in processing and expressing emotions) compared with offspring of non-stressed dams (Cratty et al, 1995; Ward et al, 2000; Salm et al, 2004). Behaviorally, Birkle's prenatal stress model results in offspring that are hyper-responsive to anxiety-provoking stimuli (e.g. increased defensive withdrawal in a novel environment and following restraint stress), an essential characteristic of the prenatal stress syndrome (Fride et al, 1988; Weinstock et al, 1988; Takahashi et al, 1988; Takahashi et al, 1990; Fride et al, 1986; Weinstock et al,1998; Cratty et al, 1995; Weinstock, 1997; Coplan et al, 1996; Ward et al, 2000; Salm et al, 2004).

In addition, Birkle has shown that PS offspring have elevated concentrations of the anxiogenic neuropeptide, CRF, as early as postnatal day 1 (P1) in the hypothalamus, and by postnatal day 14 (P14) in the amygdale (Ward et al, 2000). She also reported a neuroanatomical defect in the amygdala of this rat model of prenatal stress (Salm et al, 2004). A defect in the amygdala in these PS rats may explain how a relatively modest behavioral stressor, such as restraint, could activate potent survival signaling pathways. The amygdala receives input from brainstem serotonergic pathways and communicates with the hypothalamus. An increasing volume of basic and clinical literature supports an essential role for the amygdala in determining an individual's response to perceived external threat (Chrousous and Gold, 1992; Adolphs et al, 1995; Hariri et al, 2002). Anxiety, emotional stress and depression have each been shown to be sufficient to alter the regulation of the hypothalamic-pituitary adrenal (HPA) hormone system (Chrousous and Gold, 1992; Adolphs et al, 1995; Hariri et al, 2002; Nemeroff et al, 1984; Veith et al, 1994; Krantz et al, 1991; Jiang et al, 1996; Tafet et al, 2001). Patients with post-traumatic stress disorder (PTSD) and 
major depressive disorder (MDD) have been shown to have elevated corticotropin releasing factor (CRF) in their cerebrospinal fluid, altered circulating plasma cortisol, and an attenuation of the pituitary adrenocorticotropin hormone (ACTH) response to CRF (Chrousous and Gold, 1992; Adolphs et al, 1995; Hariri et al, 2002; Nemeroff et al, 1984; Veith et al, 1994; Krantz et al, 1991; Jiang et al, 1996; Tafet et al, 2001). Many patients with PTSD or MDD also have high baseline circulating catecholamines combined with an exaggerated increase of these neurotransmitters in response to acute emotional stress (Chrousous and Gold, 1992; Adolphs et al, 1995; Hariri et al, 2002; Nemeroff et al, 1984; Veith et al, 1994; Krantz et al, 1991; Jiang et al, 1996; Tafet et al, 2001).

\section{Previous Studies}

Adopting Birkle’s PS model, offspring were obtained by breeding virgin male (Sprague-Dawley, 250-350 g) and female rats (Sprague-Dawley, 225-250 g). Maternal stress was induced by daily subcutaneous saline injections into skin folds behind the neck $(0.9 \%, 1$ $\mathrm{ml}$ ) at different times of day and moving to a new cage from gestational days 14 through day 21, as previously reported (Kan et al, 2005; Chen et al, 2009). As adults, comparing offspring that had not been presented with a stressor, no significant differences were found using echocardiography or in vivo hemodynamics between male offspring of stressed dams and non-stressed dams. Both groups were subsequently subjected to behavioral stress at 42 and again at 49 days of post-natal age (Fig. 1). Behavioral stress was produced by restraint for two hours at room temperature in translucent plastic tubes under normal room light. The animal's heads were exposed, but they were unable to back up or turn; and they had limited lateral mobility. Therefore, studies presented here are based on comparisons between male PS rats (i.e. born from dams stressed during pregnancy) following restraint stress at 6 and 7 weeks of 
age (Stress) versus age-matched male rats (born from non-stressed dams) following the same restraint stress at 6 and 7 weeks of age (Control).

DIAGRAM OF EXPERIMENTAL PROTOCOL

Control

$$
\text { 웅 우 }
$$

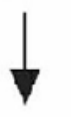

우

Pregnant

No maternal stress

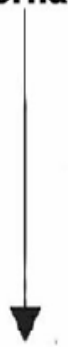

offspring

Post-natal day 42

Restraint(2hrs)

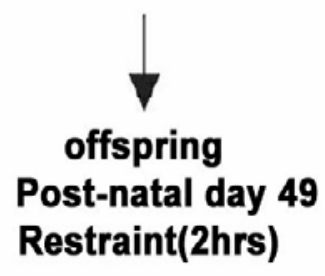

Stress

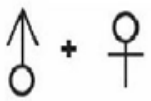

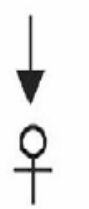

Pregnant

Maternal stress

Third trimester

Gestational days 14-21

Daily saline injection

Novel environment

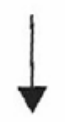

offspring

Post-natal day 42

Restraint(2hrs)

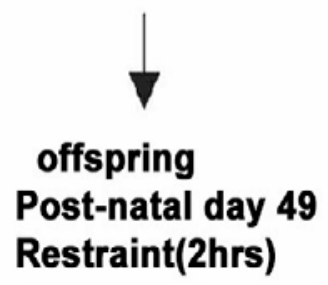

Figure 1: Flow diagram illustrating the steps involved in generating this prenatal stress animal model

Stress rats consistently revealed a decrease in systolic function, as reflected in percent fractional shortening (\% FS) by echocardiography following 2 episodes of restraint stress that were spread a week apart, as indicated in Figure 2 (Control $=61.9 \pm 2.4$ vs. Stress $=45.8 \pm$ 3.9; $\mathrm{P}<0.01 ; \mathrm{n}=12$ ). Adult rat ventricular myocytes (ARVM) isolated from Stress rats also 
revealed diminished \% FS compared with Controls, as determined by automated border detection $($ Controls $=14.1 \pm 2.9$ vs. Stress $=8.2 \pm 1.3 ; \mathrm{P}<0.01 ; \mathrm{n}=12)$ (Fig.3)

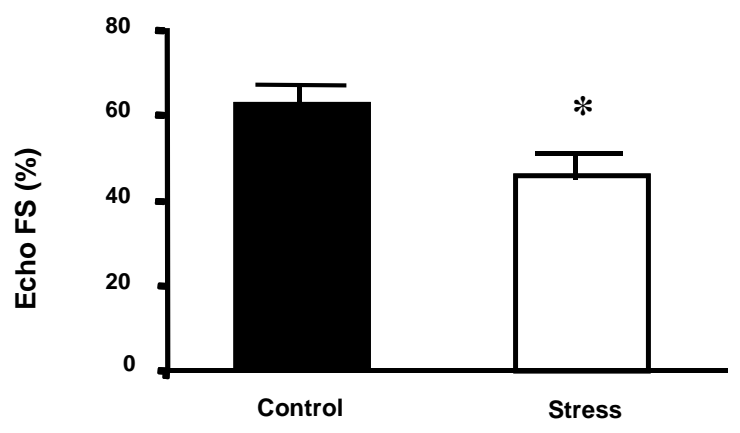

Figure 2

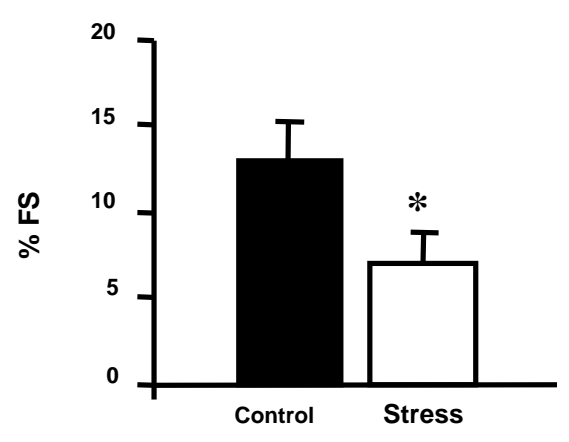

Figure 3
Bar graphs illustrating percent Fractional shortening (\%FS) determined by echocardiogram. $(61.9 \pm 2.4$ vs. $45.8 \pm$ 3.9, Control vs. Stress; $\mathrm{P}<0.01 ; \mathrm{n}=12$ )
Bar graphs illustrating contractile function of adult rat ventricular myocytes reflected as \%FS (14.1 \pm 2.9 vs. $8.2 \pm 1.3$, Control vs. Stress; $\mathrm{P}<0.01$; $\mathrm{n}=12)$

P38 MAP kinase is a central component of signaling pathways activated in response to infectious and ischemic stress (Johnson et al, 2002; Shi and Gaestel, 2002). Activation of p38 MAP kinase reversibly depressed cardiac myocyte contractility in vitro (Kan et al, 2004). The addition of the p38 MAP kinase inhibitor, SB $203580\left(10^{-5} \mathrm{M}\right)$, reversed the depression in \% FS seen in ARVM from Stress (Figure 4; $8.2 \pm 1.3$ vs. 13.3 $\pm 1.7 ; \mathrm{p}<0.01 ; \mathrm{n}=12$ ).

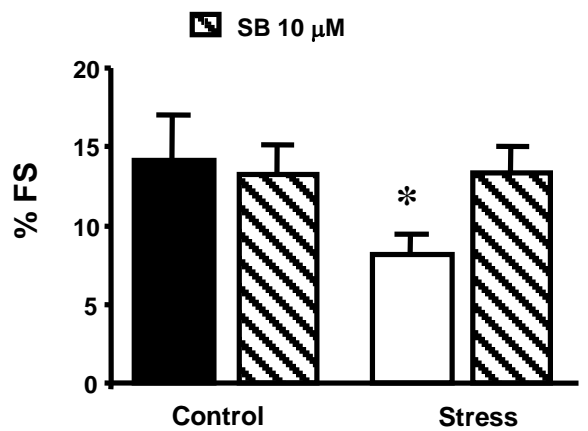

Figure 4
Bar graph illustrating the reversal of the myocardial depressant effects of Stress with the p38 MAP kinase inhibitor, SB-203580 (8.2 \pm 1.3 vs. $13.3 \pm 1.7$, Stress vs. Stress + $\mathrm{SB} ; \mathrm{p}<0.01 ; \mathrm{n}=12$ ) 
Echocardiography in patients is considered "non-invasive" because no drugs or trauma are required. However, echocardiography in animal models requires general anesthesia which may affect the results. Accordingly, our lab acquired a technique that allowed hemodynamic monitoring in freely ambulatory awake rats following initial insertion of catheters under general anesthesia. The use of the more sensitive catheter-based technique showed systolic $(+\mathrm{dp} / \mathrm{dt})$, diastolic (-dp/dt) dysfunction as well as a decrease in left ventricular systolic pressure (LVSP) in Stress versus Control offspring (Fig. 5 A-C). The p38 MAP kinase inhibitor, SB-203580 $(5 \mathrm{mg} / \mathrm{kg})$ was injected (i.p.) 24 hours before catheter surgery in accordance with previously published reports demonstrating in vivo inhibition of p38 MAP kinase enzymatic activity (Joyeux et al, 2000). Injection of SB 203580 normalized baseline $+d p / d t$, LVSP and -dp/dt in Stress compared with Control (Stress $+\mathrm{SB}$ vs. Stress; $\mathrm{p}<0.05$; Stress $+\mathrm{SB}$ vs. Control; $P=\mathrm{NS}$, for each), while having no effect on Control vs. Control +SB ( $P=$ NS; Figure 5 A-C, below).
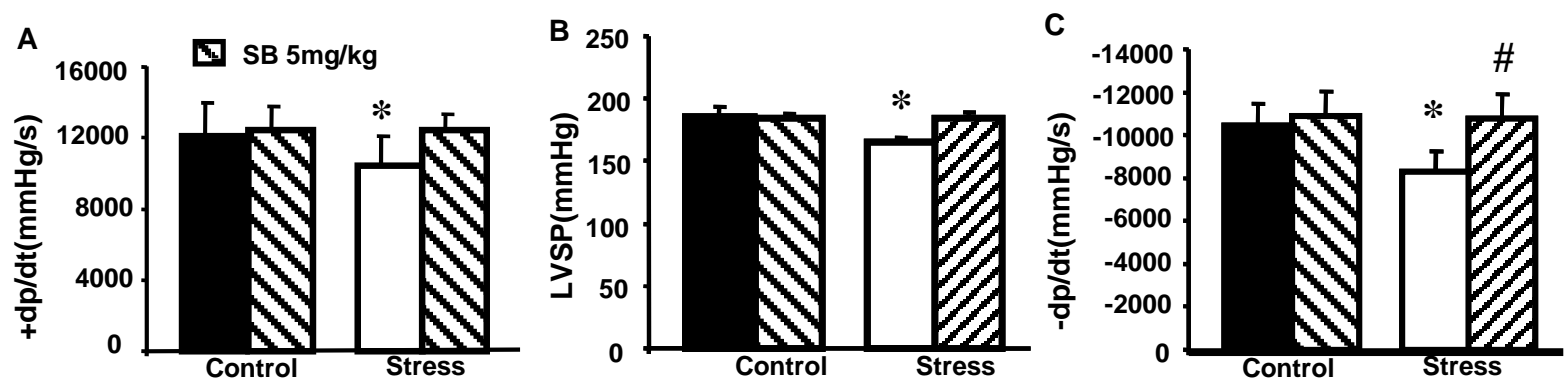

Figure 5

Bar graphs illustrating reversibility of impaired systolic (+dp/dt, A; LVSP,B) and diastolic $(-\mathrm{dp} / \mathrm{dt}, \mathrm{C})$ function by the $\mathrm{p} 38$ MAP kinas e inhibitor, SB203580 $\left({ }^{*} \mathrm{p}<0.05\right.$, Stress vs. Stress $+\mathrm{SB}, \mathrm{n}=6-8)$

The selective phosphorylation of p38 MAP kinase in Stress rats (and not Controls) and its inhibition by SB203580 was confirmed by Western blot analysis (Figure 6). We and others have 
previously reported a calcium-independent negative inotropic effect of p38 MAP kinase in cardiac myocytes (Kan et al, 2004). The calcium independence of an inotropic effect may be the result of a phosphorylation-mediated decrease in the affinity of contractile proteins for calcium (Takeishi et al, 1998; Kaye et al, 1999). Interestingly, we found an increase in Troponin I phosphorylation in Stress cardiac myocytes that was reversed by in vitro treatment with the p38 MAP kinase inhibitor, SB 203580 (Figure 6).

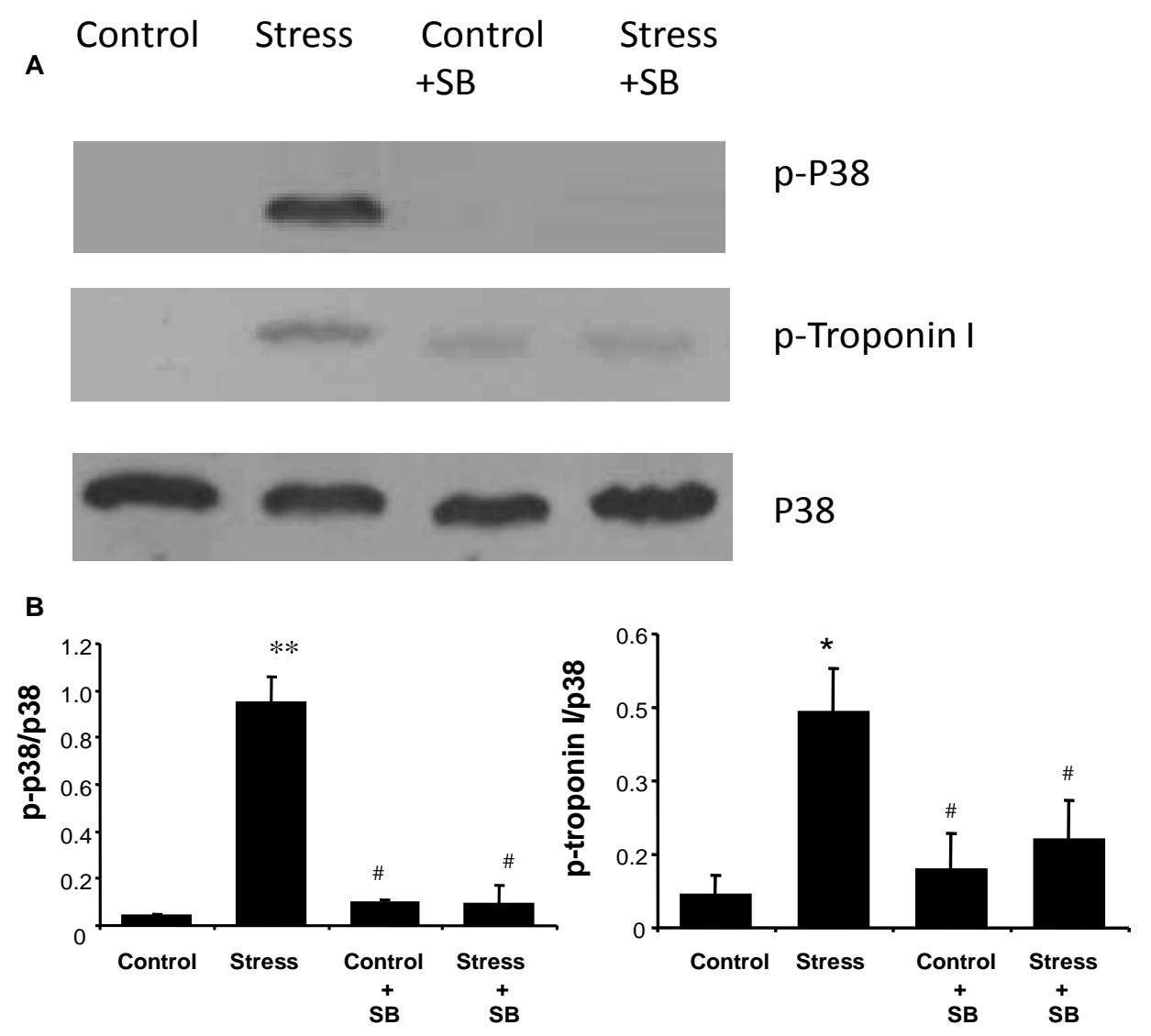

Figure 6

Representative Western Blot (A) and densitometry analysis (B) showing increased phosphorylated p38 MAP kinase and troponin I in Stress vs. Control cardiac myocytes. The p38 MAP kinase inhibitor SB reversed the increase in phosphorylation of both p38 MAP kinase and troponin I in Stress. ( ${ }^{* *} \mathrm{p}<0.01,{ }^{*} \mathrm{p}<0.05$ vs. Control; $\# \mathrm{p}<0.05$ vs. Stress) 


\section{Materials and Methods}

Stress Paradigm: Virgin six-week-old male and female Sprague Dawley rats were purchased from Hilltop Lab Animals, Inc. These animals were then housed in the CDC/NIOSH animal quarters. Male rats were used repeatedly for breeding while females experienced only one gestation. Animals were left alone for a week to allow for acclimation to their new environment before males and females were paired. The presence of a vaginal plug confirmed mating and counted as day 1 of pregnancy. The animals were then separated. Control dams were left undisturbed throughout gestation expect for normal cage changes (about once a week). Stressed dams were left undisturbed for the first two weeks of gestation except for normal cage changes. During the third and last week (days 14 to day 21), the stressed dam was removed from her cage, received a saline injection $(0.9 \%, 1 \mathrm{ml} \mathrm{sc})$ in the folds of skin on the back of the neck, was placed in a new cage and moved to a new location on the cage rack. After parturition, the dam and pups (of both groups) were left undisturbed until weaning except for normal cage changes. Pups were weaned at 3 weeks of age when they were separated from the dam and placed into cages of 2-3 same sex siblings. Only the male offspring were used for experiments. Starting at 6 weeks of age, male offspring (control and prenatally stressed) were placed in a clear, plastic restrainer for two hours and then again a week later (Fig. 1 ). During restraint the animal was unable to back up or turn and had limited lateral mobility. The day after the second restraint session, the offspring were transported from the CDC/NIOSH animal quarters to the WVU Health Science Center loading dock and then to the lab for the experimental procedures. "Stress" rats are defined as offspring of stressed dams and that were subjected to 2 successive restraint stresses. "Control" rats are offspring of dams that were undisturbed throughout pregnancy, and that were subjected to the identical restraint stress. All offspring were restrained as adults; the difference between the two groups is whether the mother was stressed during gestation. All 
experiments were performed the day of transport to the WVU Health Science Center. This study was performed in accordance with the protocol approved by both the Animal Care and Use Committees at the National Institute of Occupational Safety and Health (NIOSH) and West Virginia University.

Isolation of adult rat ventricular myocytes: Cells were isolated from the hearts of adult male Sprague-Dawley rats, as previously reported (Kan et al, 2005; Chen et al, 2009). Rats were anesthetized with pentobarbital sodium at $50 \mathrm{mg} / \mathrm{kg}$, and the hearts were removed rapidly and perfused with Krebs-Henseleit bicarbonate buffer (KHB) containing (in mM) $118.1 \mathrm{NaCl}, 3.0$ $\mathrm{KCl}$, 1.8 CaCl2, 1.2 MgSO4, 1.0 KH2PO4, 27.3 NaHCO3, 10.0 glucose, and 2.5 pyruvic acid, $\mathrm{pH} 7.4$, according to the method of Langendorff at a constant rate of $8 \mathrm{ml} / \mathrm{min}$ with a peristaltic pump. All buffer and enzyme solutions used during cell isolation were maintained at $37^{\circ} \mathrm{C}$ and preequilibrated with 95\% O2-5\% CO2. Hearts were perfused with KHB for 15 min, followed by change to low-Ca2 ${ }^{+} \mathrm{KHB}$ containing (in mM) $105.1 \mathrm{NaCl}, 3.0 \mathrm{KCl}, 0.01 \mathrm{CaCl} 2,1.2 \mathrm{MgSO} 4,1.0$ KH2PO4, 20.0 NaHCO3, 10.0 glucose, 5.0 pyruvic acid, 10.0 taurine, and 5.0 mannitol, $\mathrm{pH}$ 7.4, for an additional 10 min. Hearts were then immersed in recirculating $\mathrm{KHB}$ with low $\mathrm{Ca}^{+}$ containing Liberase TM (1.25 mg/ml; Roche Diagnostics, Mannheim, Germany) for 20 min. The ventricles were minced and placed into a 50-ml centrifuge tube, followed by dissociation of cells and debris with vigorous pipetting. The concentration of $\mathrm{Ca}^{2+}$ in $\mathrm{KHB}$ was increased in four increments $(0.05,0.4,0.8,1.2 \mathrm{mM})$. The resultant mixture was passed through $225-\mu \mathrm{m}$ nylon mesh and centrifuged at $50 \mathrm{~g}$ for $2 \mathrm{~min}$. The centrifuge procedure was repeated until the preparation was composed of at least $80 \%$ viable left ventricular myocytes. The myocytes exhibited typical striated and rod-shaped appearance when viewed by light microscope. Only 
those myocytes that were rod shaped, with striations, no blebs and were not spontaneously contracting were used for analyses (Kan et al, 2005).

Cardiac Myocyte Contractile Function: Measurements of the amplitude and velocity of unloaded single cardiac myocyte shortening and re-lengthening were made on the stage of an inverted phase-contrast microscope (Olympus, IX70-S1F2) using Myocyte Calcium Imaging/Cell Length System in which the analog motion signal was digitized and analyzed by EDGACQ edge detection software (Ionoptix Corp.), as previously reported (Kan et al, 2005; Chen et al, 2009). A coverslip with attached cells was placed in a temperature-controlled $\left(37^{0} \mathrm{C}\right)$ chamber (Series 20 Recording/Perfusion Chambers, Warner Ins. Corp.) and continuously superfused at $0.2 \mathrm{ml} / \mathrm{min}$ with $\mathrm{KHB}$ buffer. One cell per coverslip was used. Electrical field stimulation was applied at $0.5 \mathrm{~Hz}$ and about 8 volts to achieve threshold depolarization. Experiments were performed at $20 \%$ above threshold. Each cell served as its own control by continuous superfusion of buffer and drugs. Cell shortening and re-lengthening were assessed using the following indices: percent peak shortening (\%PS), maximal velocities of shortening $(+\mathrm{dl} / \mathrm{dt})$ and re-lengthening (-dl/dt), respectively.

Intracellular calcium measurements: For the measurement of changes in intracellular calcium concentrations, myocytes were incubated with $1 \mu \mathrm{M}$ Fura-2-am for 40 min, followed by washing twice and 20 min de-esterification. The cells were alternatively illuminated at 340 and $380 \mathrm{~nm}$ at a frequency of $140 \mathrm{~Hz}$. Emission for each excitation wavelength was filtered at $510 \mathrm{~nm}$. The ratio of 340/380 ratio represented the concentration in intracellular calcium concentration. Steady-state responses of ventricular myocytes to electrical-field stimulation under control and test conditions were recorded from different cells. Only rod-shaped myocytes with clear edges and no spontaneous contraction were used. Intracellular calcium changes, release and reuptake 
were assessed by change of Fura-2 ratio, Fura-2 Departure Velocity and Fur-2 Return Velocity, respectively.

To detect the response to increasing concentrations of the beta-adrenergic agonist, isoproterenol, doses of $10^{-10}, 10^{-9}, 10^{-8}$, and $10^{-7} \mathrm{M}$ were used. To measure the response to increasing extracellular calcium concentration, doses of 0.5, 1, 2, 3 and 4 mM were applied.

Response to Caffeine treatment: Cardiac myocytes were perifused with $10 \mathrm{mM}$ caffeine for 1 min, and then followed by 30-min perfusion of $\mathrm{KHB}$ alone or with $\mathrm{NAC}(0.5,1,2,3$, and $4 \mathrm{mM}$, respectively).

ATP concentration in heart tissue: Rats were anesthetized with sodium pentobarbital at $50 \mathrm{mg} / \mathrm{kg}$ and the hearts removed rapidly and perfused with phosphate-buffered saline (PBS). Trichloroacetic acid (TCA) 10\% was added to extract the ATP on ice, homogenized and centrifuged at $2000 \times \mathrm{g}$ for 10 mins. The supernatant was diluted with $50 \mathrm{mmol} / \mathrm{L}$ Tris-acetate buffer containing $2 \mathrm{mmol} / \mathrm{L}$ ethylenediaminetetraacetic acid (EDTA) (pH 7.75) to a final concentration of $0.1 \%$. Cardiac ATP content was measured using ENLITEN ATP assay system (Promega, Madison, WI, USA) according to the manufacturer's protocol.

GSH/GSSG concentration in cardiac myocytes: Isolated cardiac myocytes were incubated for 60 mins in KHB vehicle alone, or with $10^{-3} \mathrm{M}$ NAC in KHB. Myocytes were washed once with KHB, and then lysis buffer (50mM HEPES, 1Mm EDTA) was added followed by homogenization and centrifuging at $10,000 \times \mathrm{g}$ for $15 \mathrm{~min}$ at $4^{0} \mathrm{C}$. Supernatant was diluted fivefold and GSH/GSSG concentration ratio was measured by commercial glutathione assay kit (Cayman chemical, MI, USA), according to the manufacturer's protocol.

Plasma Catecholamine Concentrations: A blood sample of $900 \mu \mathrm{l}$ was collected in a microfuge tube with $100 \mu \mathrm{l}$ of $3.8 \%$ sodium citrate after rats were anesthetized by pentobarbital (50 mg/kg). 
The blood sample was centrifuged at $1,000 \times \mathrm{g}$ for $10 \mathrm{~min}$ at $4^{\circ} \mathrm{C}$. The plasma was transferred to a new centrifuge tube and stored at $-70^{\circ} \mathrm{C}$ for analysis. Plasma norepinephrine (NE) and epinephrine (EPI) were extracted with ESA plasma catecholamine kit (ESA, Inc., Chelmsford, MA 01824, USA) according to the manufacturer's instruction. Separation and quantitation of NE and EPI were accomplished by HPLC with electrochemical detection (Coulochem II, ESA, Inc., Chelmsford, MA 01824, USA). The column used was an ESA HR-80 (80 x $4.6 \mathrm{~mm})$ with the potential conditions, $200 \mathrm{mV}$ for the conditioning cell, $100 \mathrm{mV}$ for E1 and $-100 \mathrm{mV}$ for E2. The mobile phase (ESA, CAT-A-Phase II) flow was at $1.2 \mathrm{ml} / \mathrm{min}$. Data were analyzed by Waters Millennium 32 software and enabled accurate determination of noradrenaline and adrenaline. Statistical Methods: Values are expressed as means \pm SE. Myocyte data represent the means \pm SE of 15 different determinations derived from 3 individual myocytes from each of 5 different rats. Students t-test was used to compare catecholamine, ATP, GSH, GSSG, and GSH/GSSG ratio levels with 10 rats per group. Two-way ANOVA with repeated measures was used to compare the values measured in the groups. Statistical significance was accepted at the level of $\mathrm{p}<0.05$. 


\section{Results}

The two prevailing hypotheses for reversible myocardial dysfunction following behavioral stress are enhanced adrenergic stimulation and/or defective nitric oxide vascular reactivity (Bybee and Prasad, 2008; Wittstein et al, 2005; Richard, 2011). These two hypotheses are not mutually exclusive and could be related to enhanced oxidative stress. Accordingly, we compared circulating plasma catecholamine concentrations between Stress and Control the day after their second restraint (Fig 7). Both norepinephrine and epinephrine were significantly higher in the Stress compared with Control rats following exposure to the same restraint paradigm $(\mathrm{p}<0.01)$. We also compared ATP concentrations as an indication of mitochondrial function. ATP was significantly lower in Stress compared with Controls (Fig 8; $<<0.01$ ). Glutathione (GSH) is a tripeptide intracellular antioxidant that plays a central role in cellular homeostasis (Atkuri et al, 2007). We compared the relative oxidized (GSSG) and reduced (GSH) states of glutathione to assess the redox status of our model the day after their second restraint (Fig 9). Stress revealed significantly higher oxidized glutathione (GSSH) and lower ratio of GSH/GSSG compared with Controls $(\mathrm{p}<0.01)$.
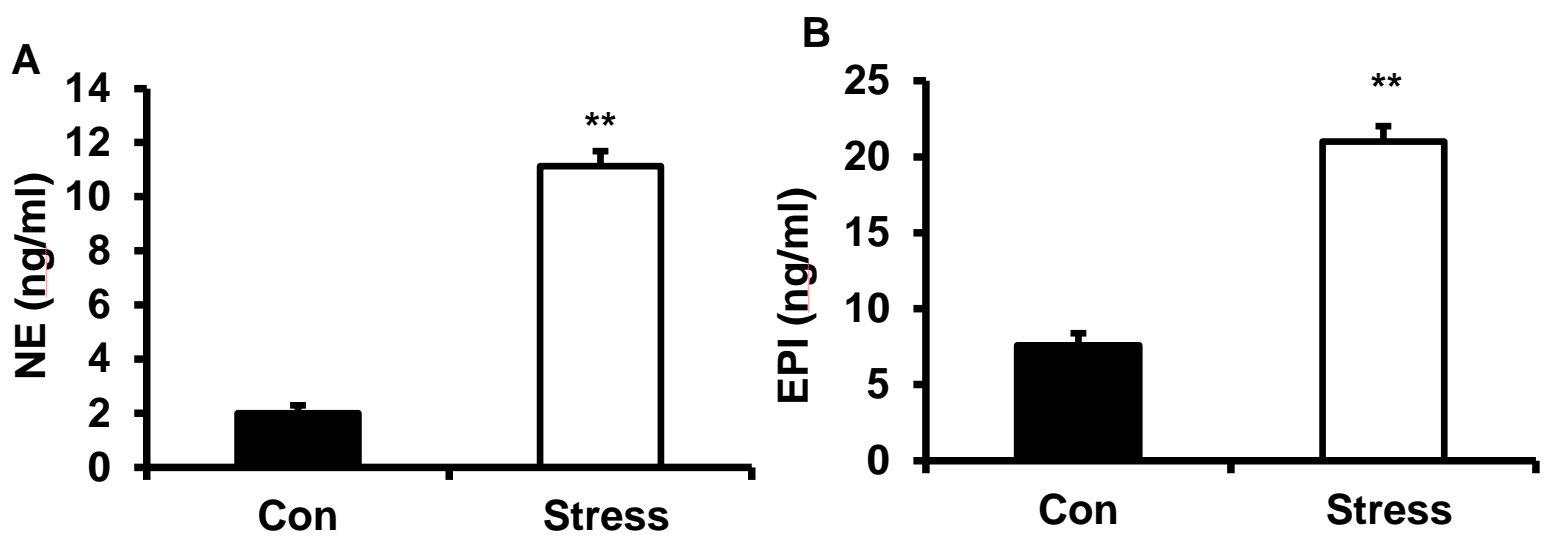

Figure 7: Bar graphs depicting plasma norepinephrine (NE) $(1.99 \pm 0.29$ vs. $11.13 \pm 0.55 \mathrm{ng} / \mathrm{ml}$, con vs. stress, $\left.{ }^{* *} \mathrm{p}<0.01 ; \mathrm{n}=10\right)(\mathrm{A})$; and epinephrine (EPI) (7.60 \pm 0.80 vs. $21.01 \pm 1.02$, Con vs. Stress, $* * \mathrm{p}<0.01 ; \mathrm{n}=10)(\mathrm{B})$. 


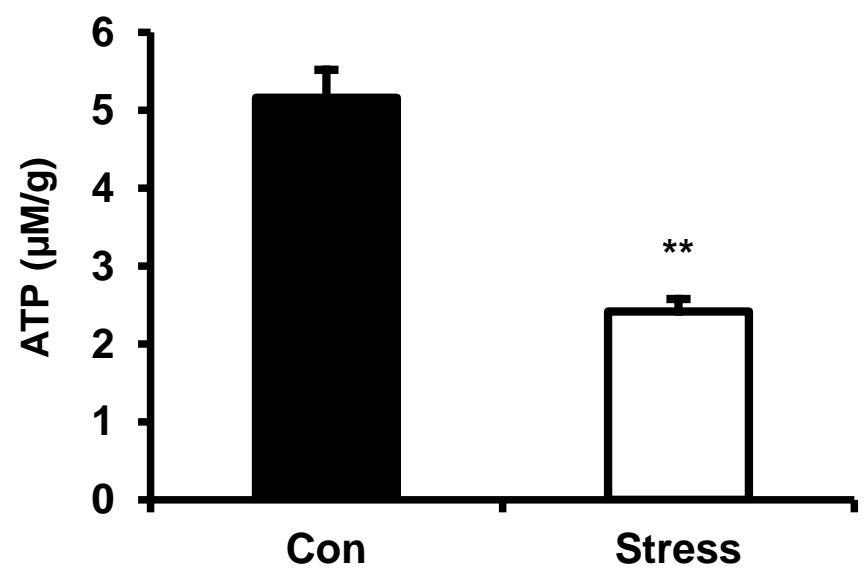

Figure 8: Bar graph depicting ATP levels measured in extracts from homogenized whole hearts (5.16 \pm 0.36 vs. $2.42 \pm 0.16 \mu \mathrm{M} / \mathrm{g}$ total protein, Con vs. Stress, ${ }^{* *} \mathrm{p}<0.05$; $n=10)$.
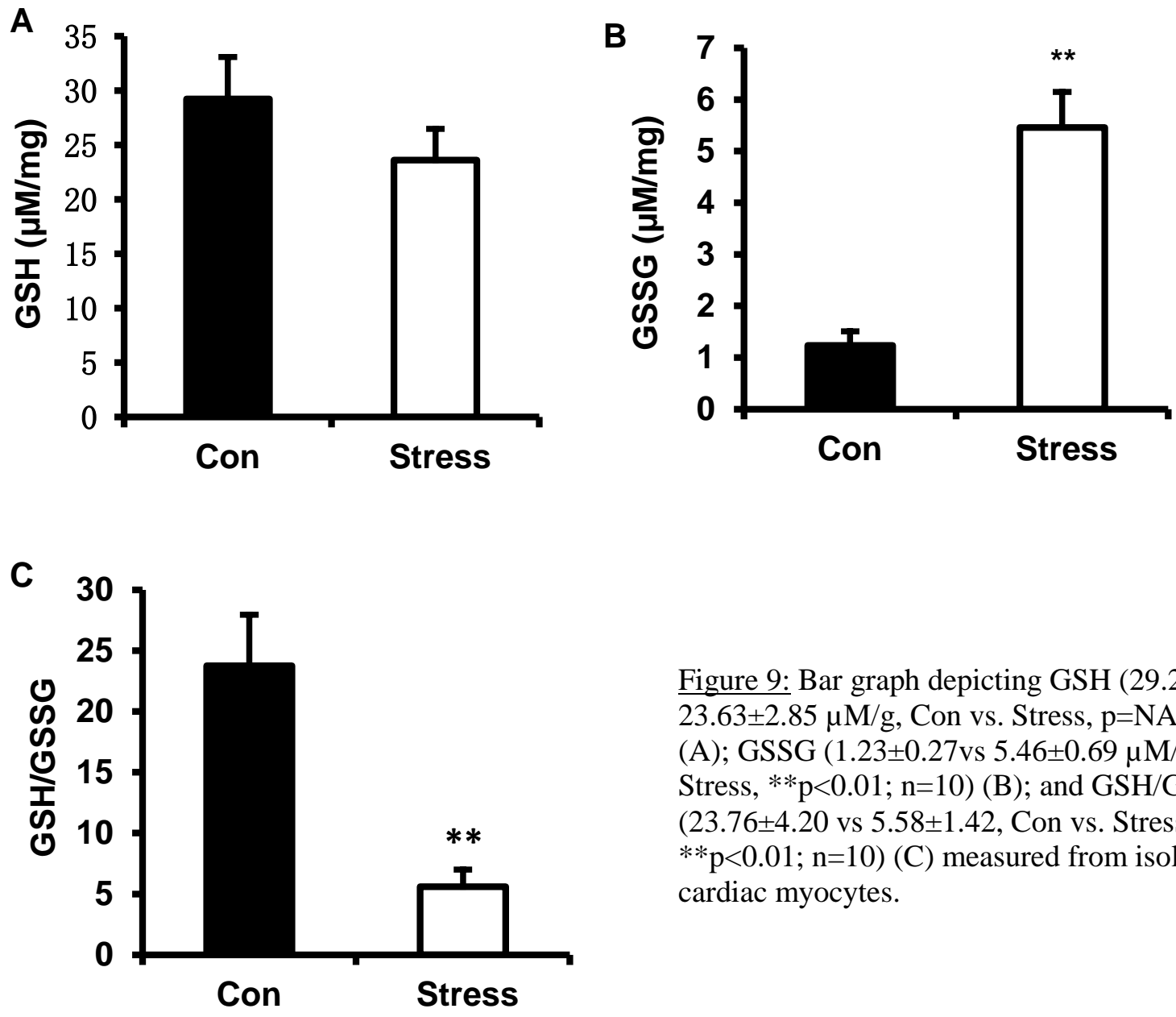

Figure 9: Bar graph depicting GSH (29.24 $\pm 3.84 \mathrm{vs}$ $23.63 \pm 2.85 \mu \mathrm{M} / \mathrm{g}$, Con vs. Stress, $\mathrm{p}=\mathrm{NA}$; $\mathrm{n}=10$ ) (A); GSSG (1.23 $\pm 0.27 v s 5.46 \pm 0.69 \mu \mathrm{M} / \mathrm{g}$, Con vs. Stress, $\left.{ }^{* *} \mathrm{p}<0.01 ; \mathrm{n}=10\right)(\mathrm{B})$; and GSH/GSSG ratio $(23.76 \pm 4.20$ vs $5.58 \pm 1.42$, Con vs. Stress, $\left.{ }^{* *} \mathrm{p}<0.01 ; \mathrm{n}=10\right)(\mathrm{C})$ measured from isolated cardiac myocytes. 
$\mathrm{N}$-acetylcysteine (NAC) has been shown to raise intracellular concentrations of reduced glutathione by providing an additional source of cysteine as a precursor (Choy et al, 2010; Han et al, 2010; Kerksick and Willoughby, 2005), to reduce disulfide bonds (Harada et al, 2004), and scavenge free radicals (Kelly, 1998). Accordingly, we compared the inotropic effects of NAC on Stress and Controls (Fig 10). NAC $\left(10^{-3}\right)$ completely normalized the decrease in \% PS in Stress to Control values without affecting Controls $(\mathrm{p}<0.01)$.

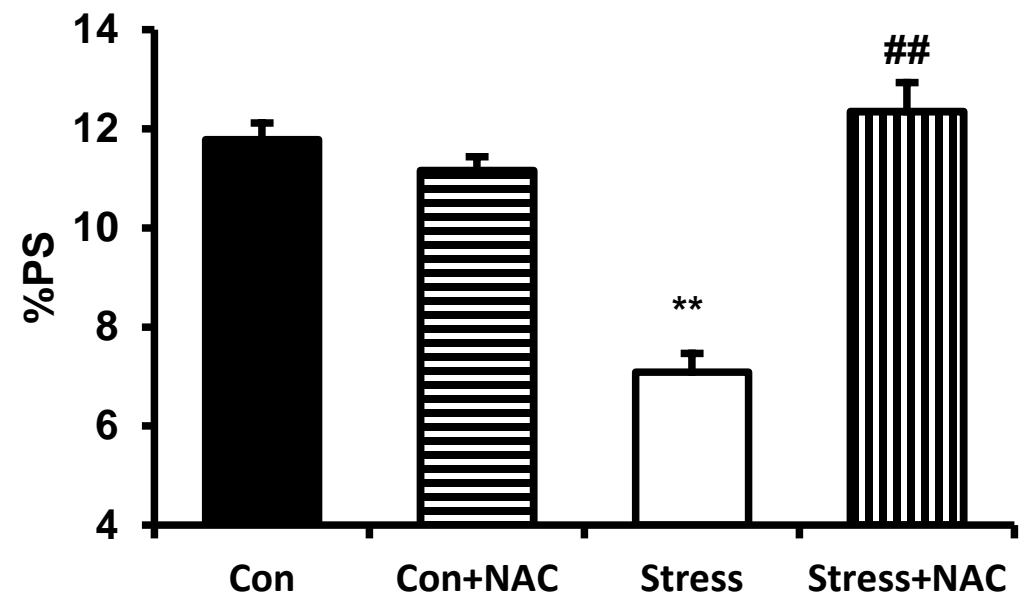

Figure 10: Baseline percent peak shortening (\%PS) in control and stress myocytes with and without the addition of NAC $\left(10^{-3} \mathrm{M}\right)(11.78 \pm 0.34$ vs. $7.09 \pm 0.38 \%$, Con vs. Stress, $* * \mathrm{p}<0.01 ; 7.09 \pm 0.38$ vs. $12.34 \pm 0.59$ $\%$, Stress vs. Stress+NAC, \#\#p $<0.01$; $\mathrm{n}=15)$.

Blunted/attenuated inotropic response to adrenergic stimulation is one of the hallmark characteristics of virtually all cardiomyopathies (Kan and Finkle, 2003). Our laboratory has previously shown in both in vitro and in vivo experiments that Stress shares this cardiomyopathy feature. (Kan et al, 2005; Chen et al, 2009). Accordingly, we sought to determine if adding NAC would reverse the blunted inotropic responses of Stress to the beta adrenergic agonist, isoproterenol (ISO) (Fig 11). NAC completely normalized the concentration-response curves to increasing concentrations of ISO for total \% PS, $+\mathrm{dl} / \mathrm{dt}$ and $-\mathrm{dl} / \mathrm{dt}$ ( $\mathrm{p}<0.01$ for each). 


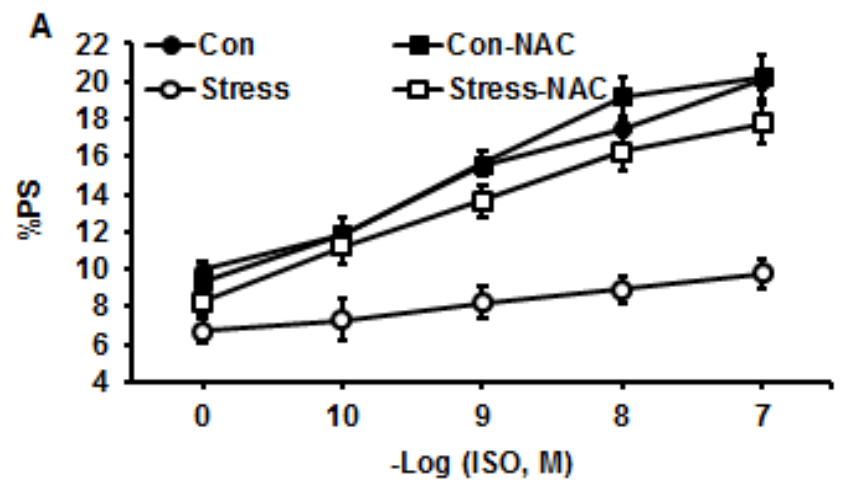

C

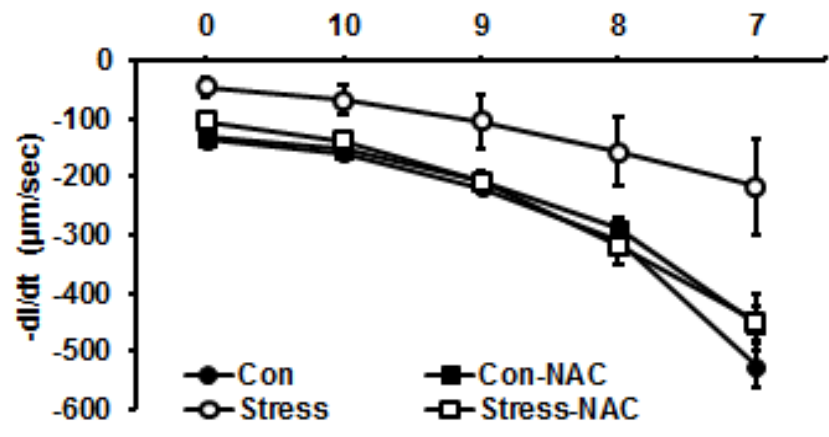

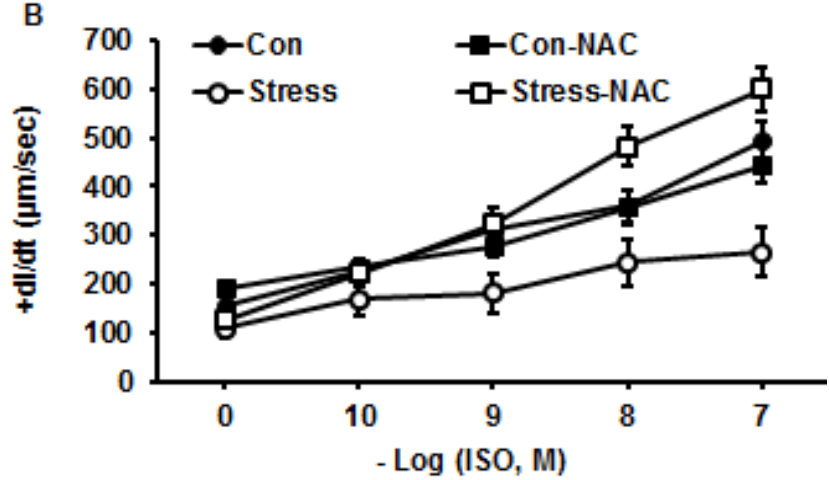

Figure 11: Graphic depiction of the response of cardiac myocytes to increasing concentrations of the beta-adrenergic agonist, isoproterenol, alone and with incubation with NAC $\left(10^{-3} \mathrm{M}\right)$ on percent peak shortening (\%PS) (Con vs. Stress, $\mathrm{p}<0.01$; Stress vs. Stress + NAC, $\mathrm{p}<0.01 ; \mathrm{n}=15)(\mathrm{A})$, positive inotropy $(+\mathrm{dl} / \mathrm{dt})($ Con vs. Stress, $\mathrm{p}<0.01$; Stress vs. Stress + NAC, $\mathrm{p}<0.01 ; \mathrm{n}=15)(\mathrm{B})$, and negative inotropy ($\mathrm{dl} / \mathrm{dt}$ ) (Con vs. Stress, $\mathrm{p}<0.01$; Stress vs. Stress + NAC, $\mathrm{p}<0.01 ; \mathrm{n}=15)(\mathrm{C})$.

Isoproterenol mediates its inotropic effects through regulation of intracellular calcium signaling in cardiac myocytes through stimulation of the beta-receptor (Kan and Finkel, 2003). Accordingly, we measured the effect of NAC on intracellular $\mathrm{Ca}^{2+}$ responses to ISO (Fig 12). We confirmed that the NAC mediated inotropic effects were associated with reversing/normalizing the blunted $\mathrm{Ca}^{2+}$ responses to ISO $(\mathrm{p}<0.01)$. 

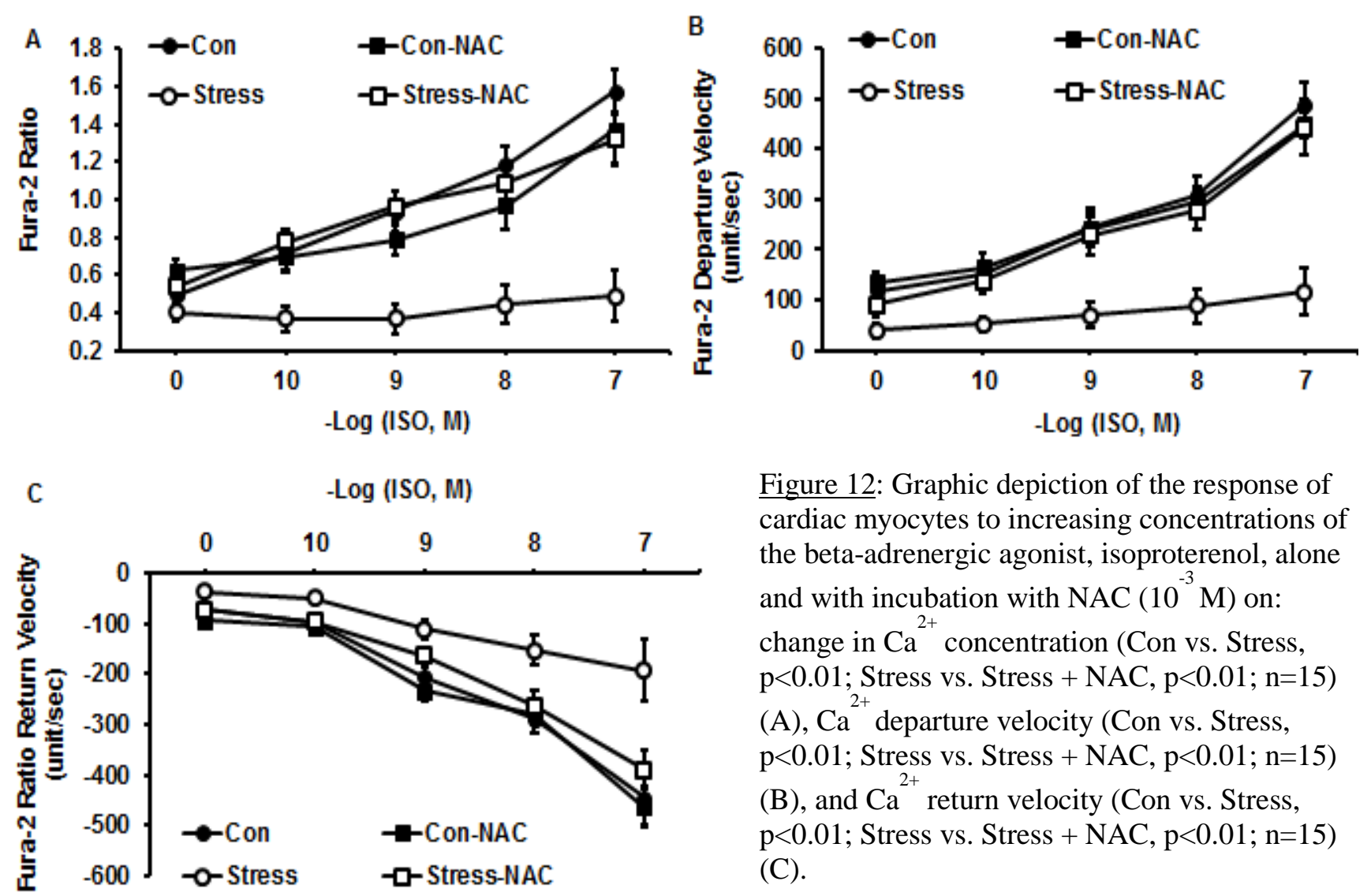

Figure 12: Graphic depiction of the response of cardiac myocytes to increasing concentrations of the beta-adrenergic agonist, isoproterenol, alone and with incubation with NAC $\left(10^{-3} \mathrm{M}\right)$ on: change in $\mathrm{Ca}^{2+}$ concentration (Con vs. Stress, $\mathrm{p}<0.01$; Stress vs. Stress + NAC, $\mathrm{p}<0.01 ; \mathrm{n}=15$ ) (A), $\mathrm{Ca}^{2+}$ departure velocity (Con vs. Stress, $\mathrm{p}<0.01$; Stress vs. Stress + NAC, $\mathrm{p}<0.01 ; \mathrm{n}=15$ )

(B), and $\mathrm{Ca}^{2+}$ return velocity (Con vs. Stress, $\mathrm{p}<0.01$; Stress vs. Stress + NAC, $\mathrm{p}<0.01 ; \mathrm{n}=15$ ) (C).

Chen et al (2012) has recently reported that NAC reversed/normalized the blunted inotropic response of cardiac myocytes to extracellular calcium using a genetic cardiomyopathy model. We now report that NAC similarly reversed/normalized the blunted inotropic and intracellular calcium responses to extracellular calcium (Figs $13 \& 14$; $<<0.01$, for each). 

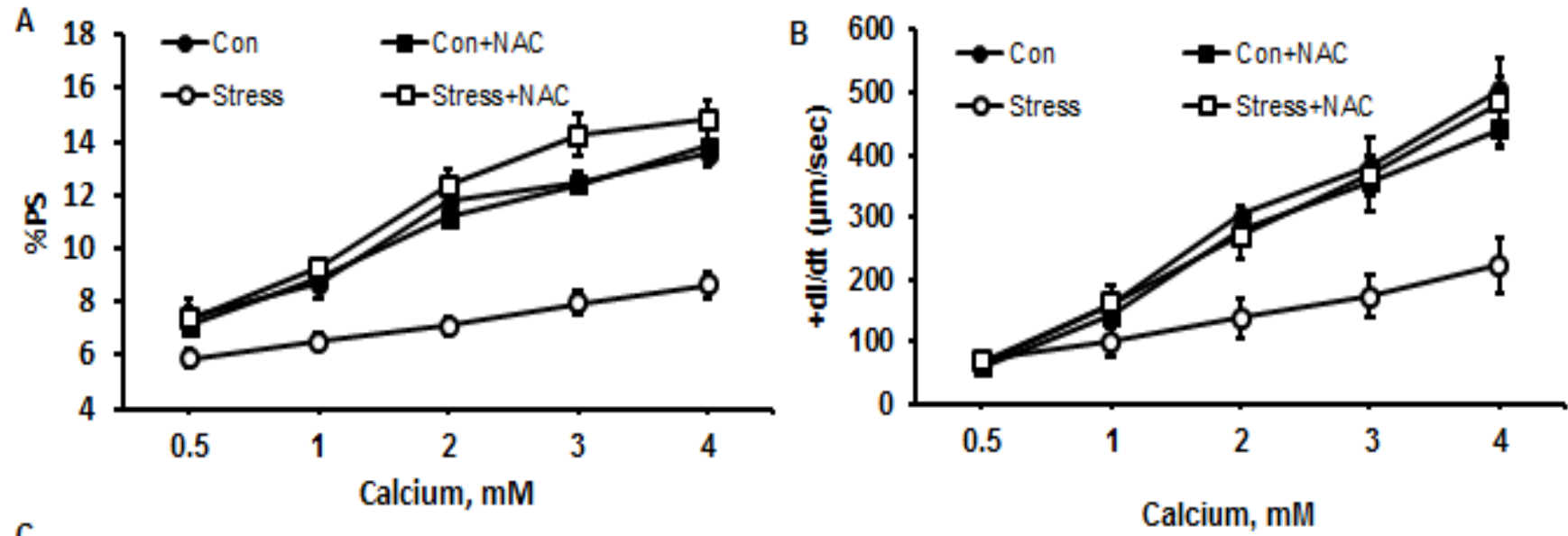

C

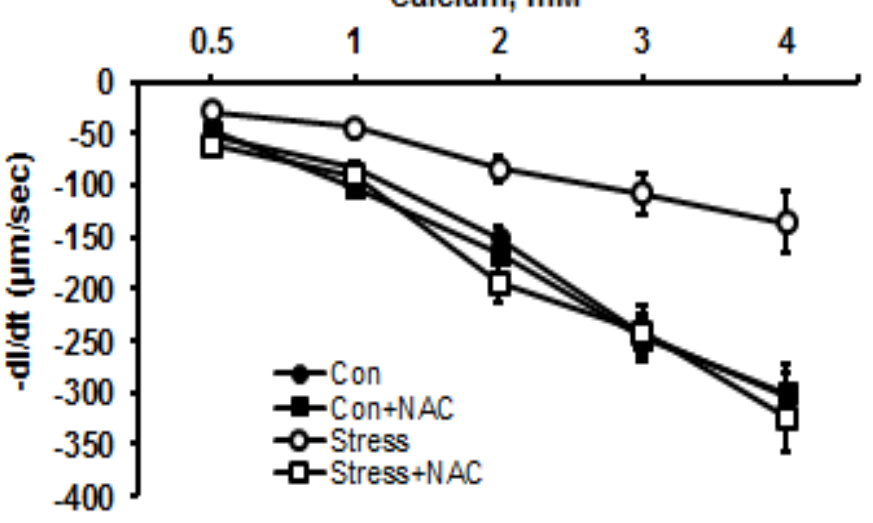

Figure 13: Graphic depiction of the response of cardiac myocytes to increasing concentrations of extracellular $\mathrm{Ca}^{2+}$ alone and with incubation with NAC $\left(10^{-3} \mathrm{M}\right)$ on percent peak shortening (\%PS) (Con vs. Stress, $\mathrm{p}<0.01$; Stress vs. Stress + NAC, $\mathrm{p}<0.01 ; \mathrm{n}=15$ ) (A), positive inotropy $(+\mathrm{dl} / \mathrm{dt})($ Con vs. Stress, $\mathrm{p}<0.01$; Stress vs. Stress + NAC, $\mathrm{p}<0.01 ; \mathrm{n}=15$ ) (B), and negative inotropy (-dl/dt) (Con vs. Stress, $\mathrm{p}<0.01$; Stress vs. Stress + NAC, $\mathrm{p}<0.01 ; \mathrm{n}=15)(\mathrm{C})$. 

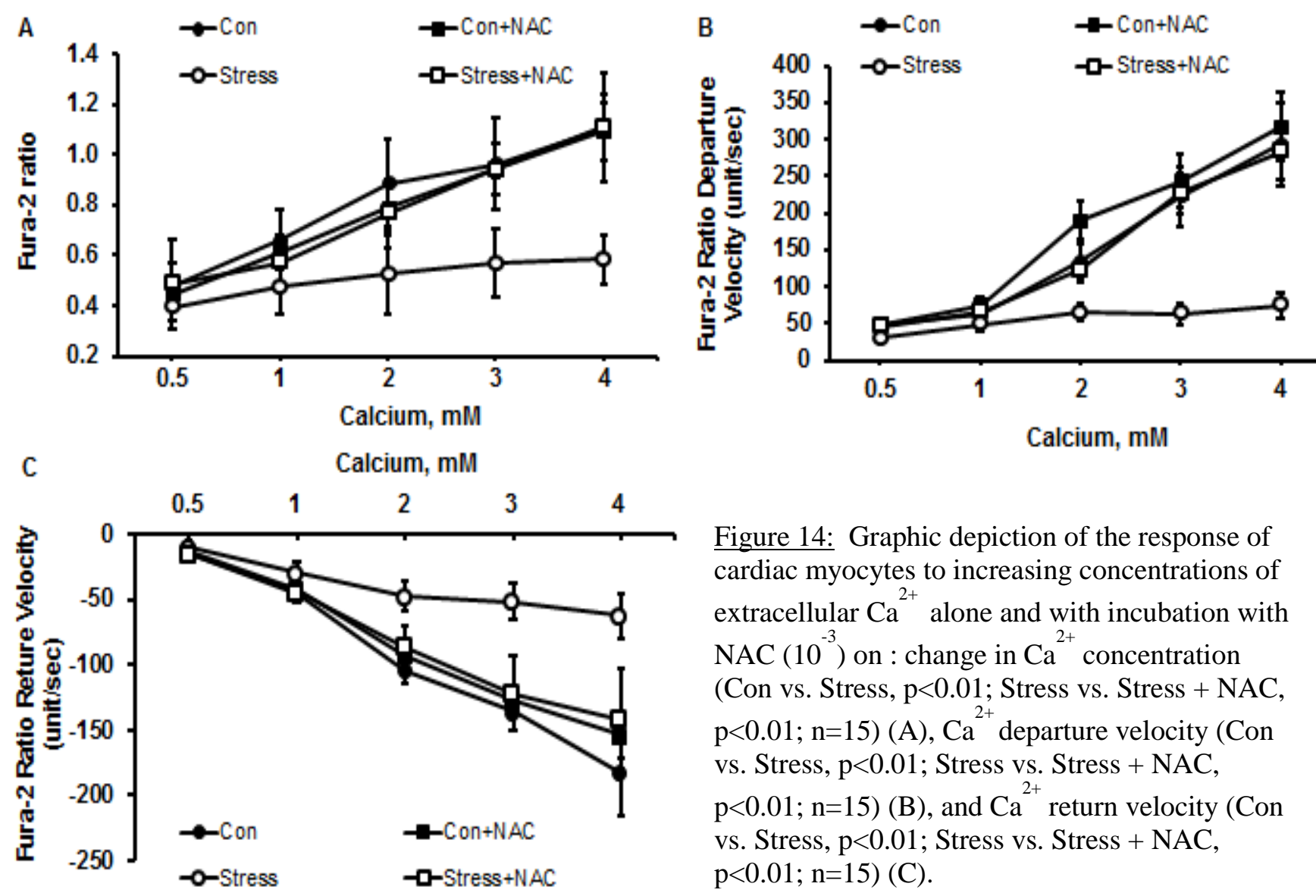

Figure 14: Graphic depiction of the response of cardiac myocytes to increasing concentrations of extracellular $\mathrm{Ca}^{2+}$ alone and with incubation with NAC $\left(10^{-3}\right)$ on : change in $\mathrm{Ca}^{2+}$ concentration (Con vs. Stress, $\mathrm{p}<0.01$; Stress vs. Stress + NAC, $\mathrm{p}<0.01 ; \mathrm{n}=15)(\mathrm{A}), \mathrm{Ca}^{2+}$ departure velocity (Con vs. Stress, $\mathrm{p}<0.01$; Stress vs. Stress $+\mathrm{NAC}$, $\mathrm{p}<0.01 ; \mathrm{n}=15)(\mathrm{B})$, and $\mathrm{Ca}^{2+}$ return velocity (Con vs. Stress, $\mathrm{p}<0.01$; Stress vs. Stress + NAC, $\mathrm{p}<0.01 ; \mathrm{n}=15)(\mathrm{C})$.

It has been previously proposed that NAC mediates inotropic effects through the sarcoplasmic reticulum calcium release channel (SRCRC; RYR2) (Finkel et al, 1993; Chen et al, 2012). Caffeine is well-known to cause release of SR calcium (Gaburjakova \& Gaburjakova, 2006). Accordingly, we compared the inotropic and calcium regulatory effects of caffeine on Stress and Controls (Figs 15-26). The addition of caffeine decreased the \% PS in cardiac myocytes from both Stress and Control. (Fig 16A). However, the decrease was greater in the Stress compared with Controls (16Fig A\&B; $<<0.01$ ). The same relatively greater effect of caffeine on Stress compared with Controls was seen in $+\mathrm{dl} / \mathrm{dt}$ and -dl/dt (Figs 17 \&18; p<0.01). Caffeine also had a greater effect on the release of intracellular calcium in response to electrical field stimulation in Stress compared with Controls (Figs 19-21). This difference was true when 
measuring total, as well as, rate of release or reuptake of intracellular calcium ( $<<0.01$, for each). The duration of the inotropic and calcium regulatory effects of caffeine were also longer-lasting in the Stress compared with Control myocytes (Figs 22\&23; $<<0.01$, for each). This was true for $\% \mathrm{PS},+\mathrm{dl} / \mathrm{dt}$, $-\mathrm{dl} / \mathrm{dt}$, total calcium, calcium release and reuptake velocities $(\mathrm{p}<0.01$, for each).

The addition of NAC following caffeine significantly reversed both the inotropic and calcium regulatory effects of caffeine alone (Fig 24-26). This was true for \%PS, +dl/dt, -dl/dt, total calcium, calcium release and reuptake velocities ( $\mathrm{p}<0.01$, for each). NAC had no effect on GSH, GSSG or GSH/GSSG in either Stress or Controls (Fig 27 A-C).

A

Cardiac Myocytes Contraction

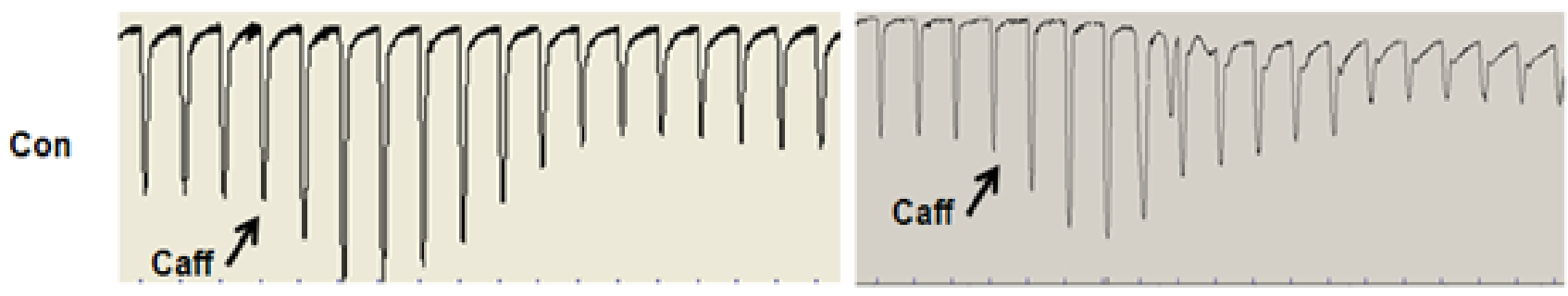

Stress

B

Intracellular Calcium Concentration

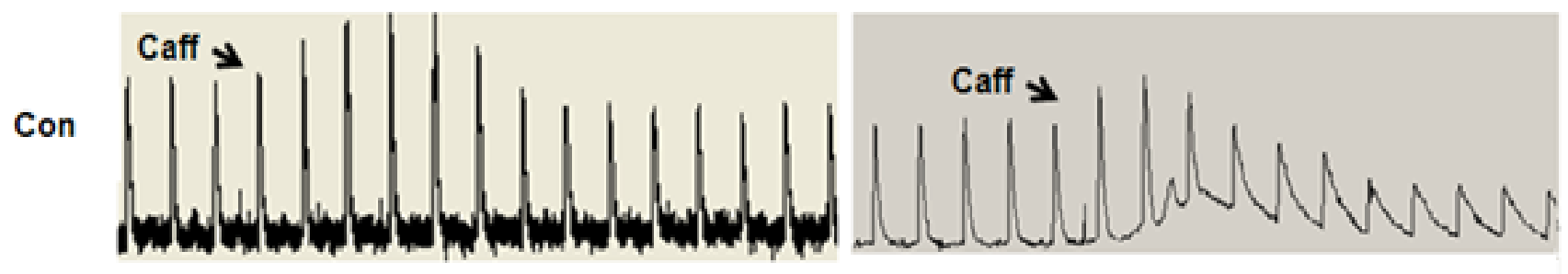

Figure 15: Tracing of control and stress cardiac myocytes contraction right before and after exposure to caffeine (10mM) (A) and the tracing of intracellular $\mathrm{Ca}^{2+}$ concentration (B). 

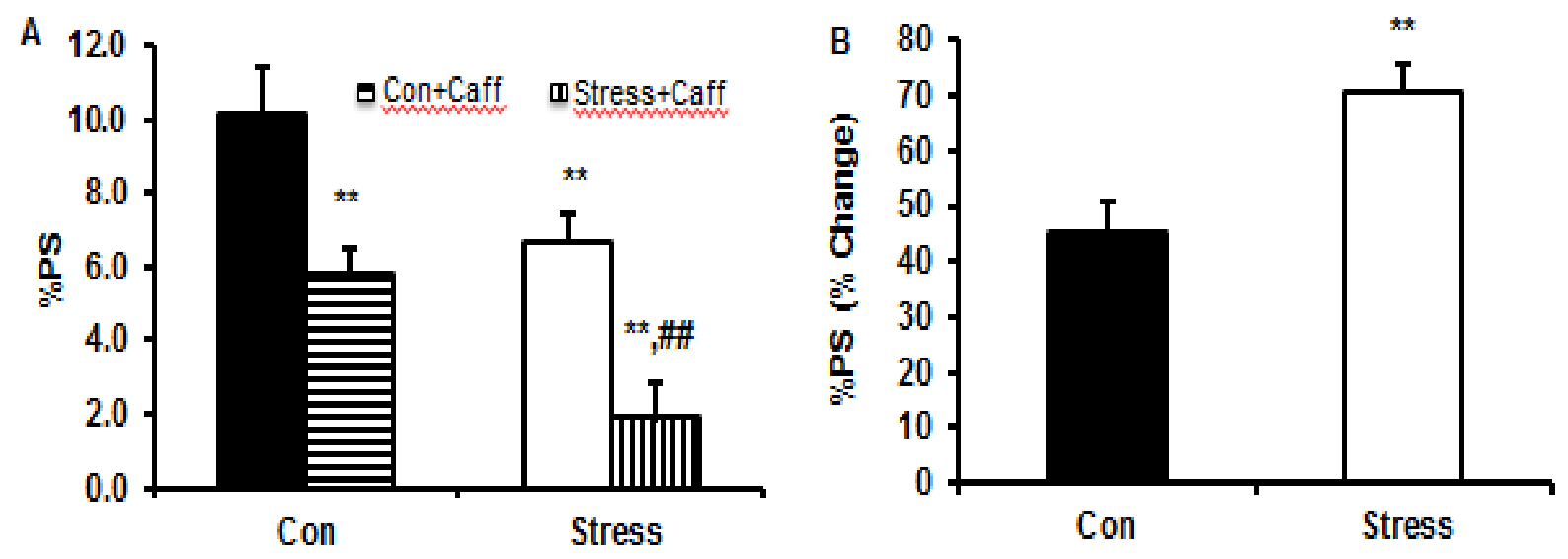

Figure 16. Bar graph depicting \% PS of control and stress cardiac myocytes before and after exposure to caffeine $(10 \mathrm{mM})\left(10.10 \pm 1.27\right.$ vs. $6.68 \pm 0.77$, Con vs. Stress, ${ }^{* *} \mathrm{p}<0.01 ; 10.10 \pm 1.27$ vs. $5.8 \pm 0.66$, Con vs. Con+Caff, ** $\mathrm{p}<0.01 ; 6.68 \pm 0.77$ vs. $1.88 \pm 0.93$, Stress vs. Stress+Caff, \#\# $\mathrm{p}<0.01 ; \mathrm{n}=15)(\mathrm{A})$; and percentage change of control and stress cardiac myocytes after exposure to caffeine $(10 \mathrm{mM})(44.99 \pm 5.75$ vs $70.60 \pm 5.09$ $\%$, Con vs. Stress, $\left.{ }^{* *} \mathrm{p}<0.01 ; \mathrm{n}=15\right)(\mathrm{B}) .{ }^{* *} \mathrm{p}<0.01$ vs. Control; $\# \mathrm{p}<<0.01$ vs. Stress.
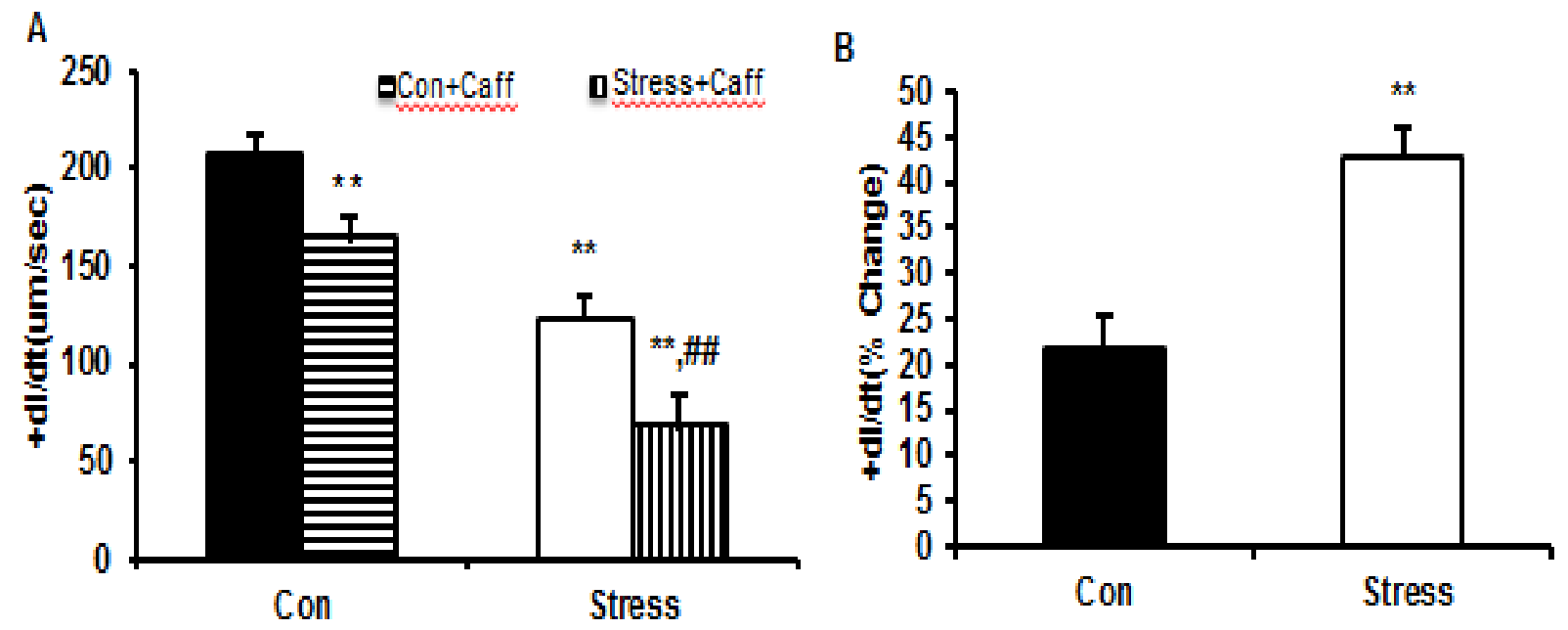

Figure 17: Bar graph depicting positive inotropy $(+\mathrm{dl} / \mathrm{dt})$ of control and stress cardiac myocytes before and after exposure to caffeine $(10 \mathrm{mM})\left(206.98 \pm 10.41\right.$ vs. $122.74 \pm 11.24$, Con vs. Stress, ${ }^{* *}$ $\mathrm{p}<0.01 ; 206.98 \pm 10.41$ vs. $164.10 \pm 11.08$, Con vs. Con+Caff, ** $\mathrm{p}<0.01 ; 122.74 \pm 11.24$ vs.

$67.98 \pm 16.04$, Stress vs. Stress+Caff, \#\# $\mathrm{p}<0.01 ; \mathrm{n}=15$ ) (A); and percentage change of control and stress cardiac myocytes after exposure to caffeine $(10 \mathrm{mM})(21.59 \pm 3.77$ vs $40.96 \pm 3.82 \%$, Con vs. Stress, $\left.{ }^{* *} \mathrm{p}<0.01 ; \mathrm{n}=15\right)(\mathrm{B}) .{ }^{* *} \mathrm{p}<0.01$ vs. Control; $\# \# \mathrm{p}<0.01$ vs. Stress. 

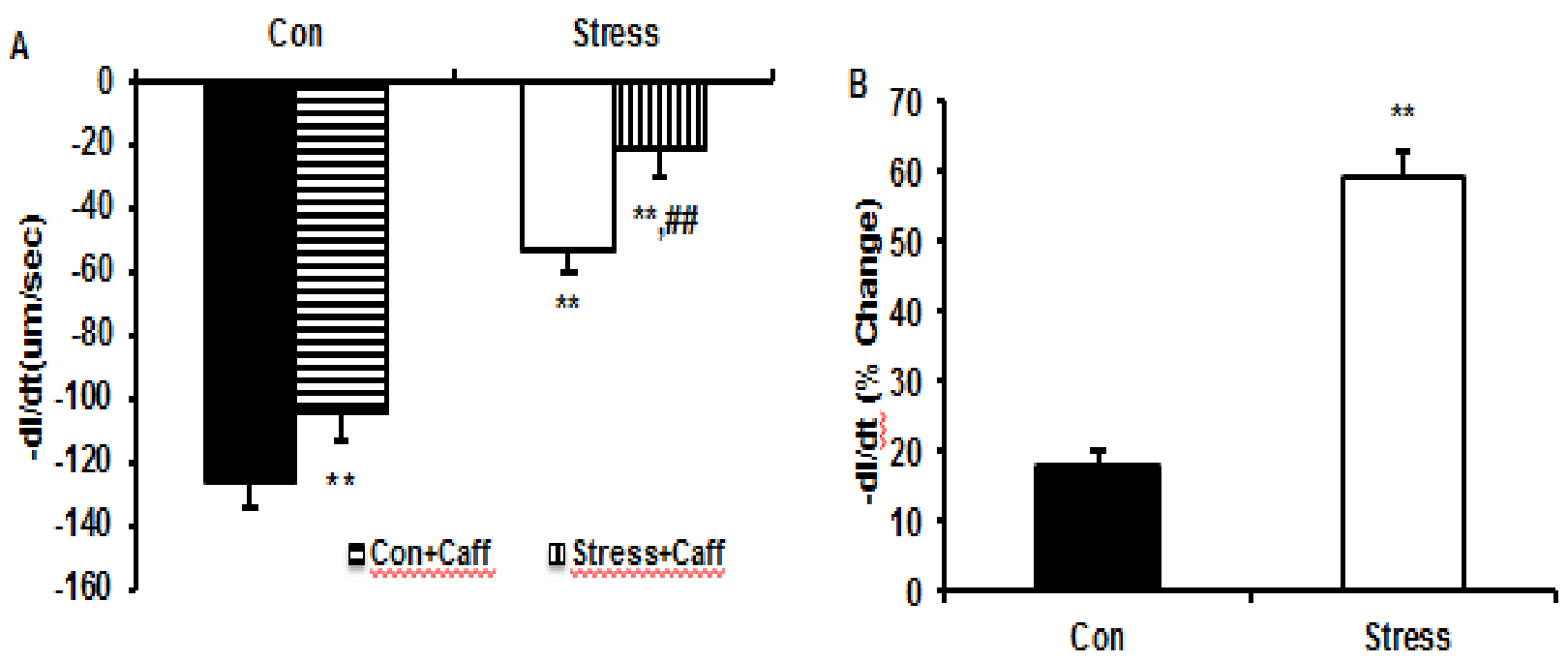

Figure 18: Bar graph depicting negative inotropy (-dl/dt) of control and stress cardiac myocytes before and after exposure to caffeine $(10 \mathrm{mM})\left(-126.56 \pm 8.06\right.$ vs. $-53.28 \pm 6.84$, Con vs. Stress, ${ }^{* *} \mathrm{p}<0.01 ;-126.56 \pm 8.06$ vs. $-104.23 \pm 9.32$, Con vs. Con+Caff, $* * \mathrm{p}<0.01 ;-53.28 \pm 6.84$ vs. $-21.53 \pm 8.24$, Stress vs. Stress + Caff, \#\# $\mathrm{p}<0.01 ; \mathrm{n}=15)(\mathrm{A})$; and percentage change of control and stress cardiac myocytes after exposure to caffeine $(10 \mathrm{mM})\left(17.78 \pm 2.17\right.$ vs. $59.41 \pm 3.46 \%$, Con vs. Stress, $\left.{ }^{* *} \mathrm{p}<0.01 ; \mathrm{n}=15\right)(B) .{ }^{* *} \mathrm{p}<0.01$ vs. control; $\# \# \mathrm{p}<0.01$ vs. stress.
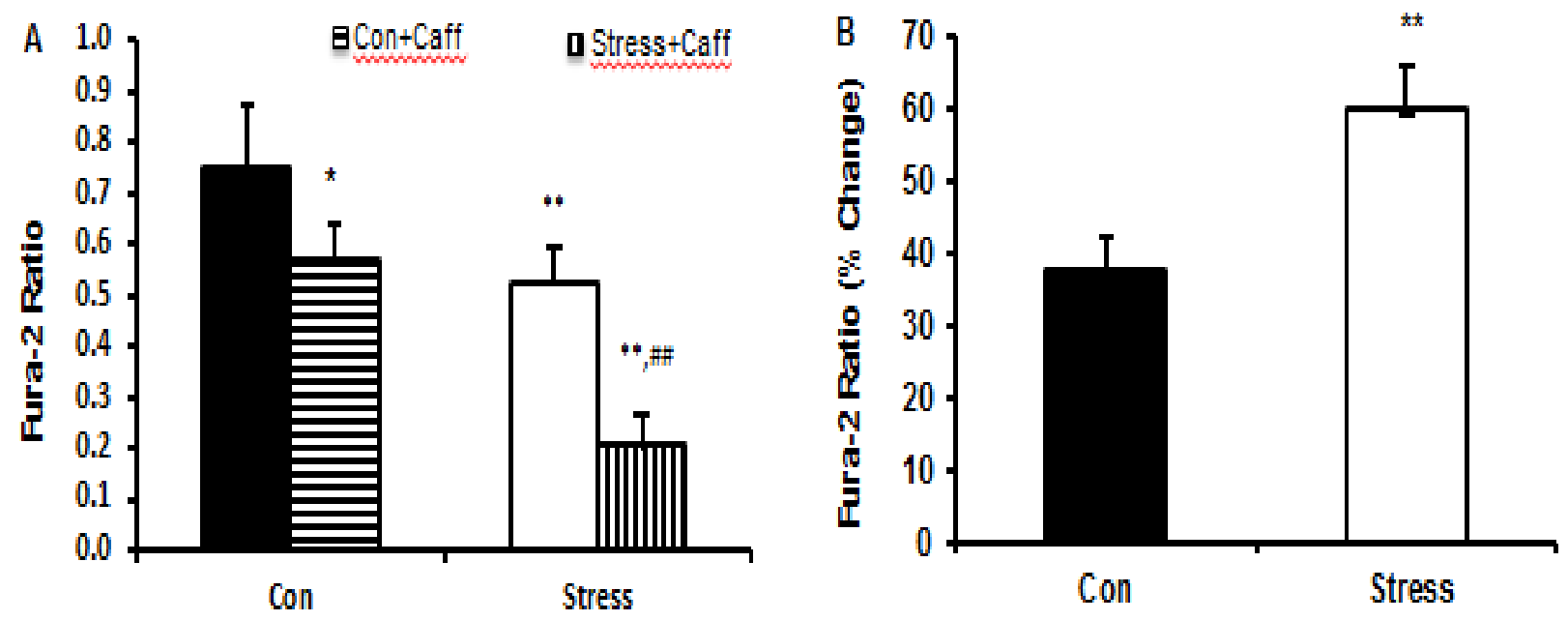

Figure 19: Bar graph depicting change in calcium concentration in control and stress cardiac myocytes before and after exposure to caffeine $(10 \mathrm{mM})(0.75 \pm 0.12 \mathrm{vs} .0 .53 \pm 0.07$, Con vs. Stress, ${ }^{* *} \mathrm{p}<0.01 ; 0.75 \pm 0.12$ vs. $0.57 \pm 0.07$, Con vs. Con+Caff, ${ }^{*} \mathrm{p}<0.05 ; 0.53 \pm 0.07$ vs. $0.20 \pm 0.06$, Stress vs. Stress+Caff, \#\# $\mathrm{p}<0.01 ; \mathrm{n}=15)(\mathrm{A})$; and percentage change of control and stress cardiac myocytes after exposure to caffeine $(10 \mathrm{mM})(37.72 \pm 5.68$ vs $60.02 \pm 1.04 \%$, Con vs. Stress, $\left.{ }^{* *} \mathrm{p}<0.01 ; \mathrm{n}=15\right)(\mathrm{B}) .{ }^{*} \mathrm{p}<0.05$ vs. control; ${ }^{* *} \mathrm{p}<0.01$ vs. control; $\# \# \mathrm{p}<0.01$ vs. stress. 

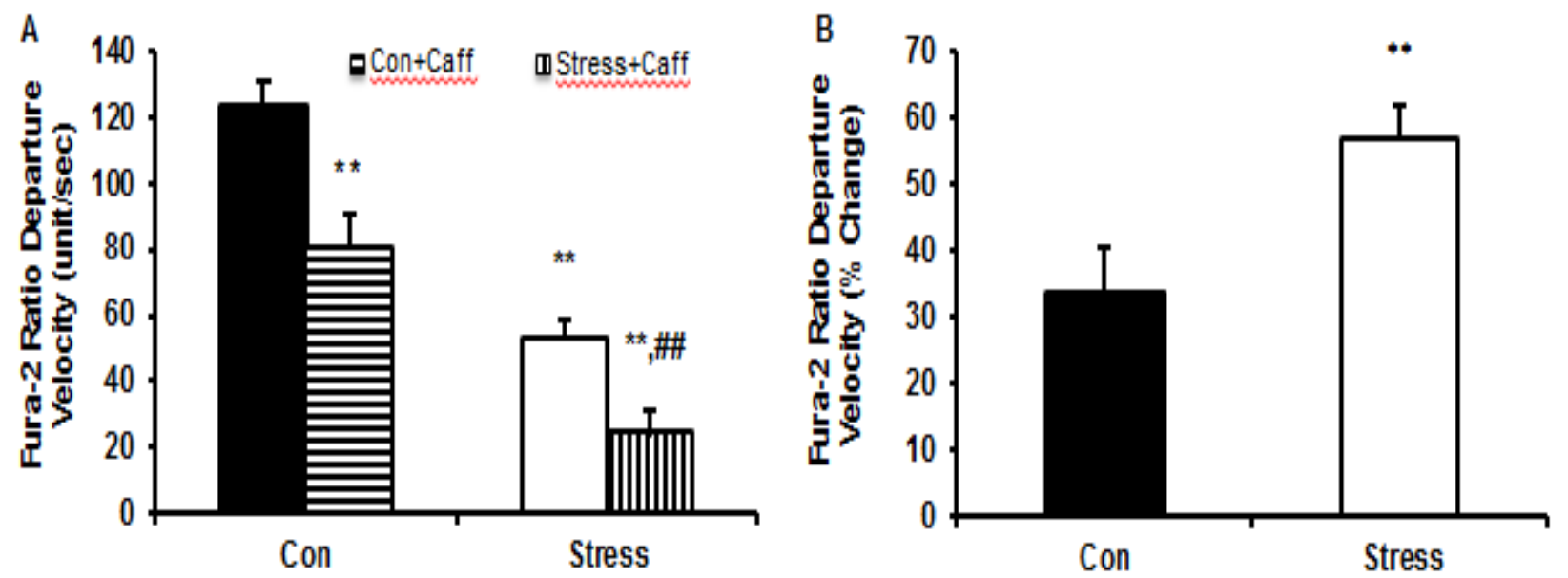

Figure 20: Bar graph depicting calcium departure velocity in control and stress cardiac myocytes before and after exposure to caffeine $(10 \mathrm{mM})\left(123.60 \pm 6.70\right.$ vs. $52.90 \pm 5.64$, Con vs. Stress, ${ }^{* *} \mathrm{p}<0.01$; $123.60 \pm 6.70$ vs. $80.54 \pm 8.91$, Con vs. Con+Caff, $* * \mathrm{p}<0.01$; $52.90 \pm 5.64$ vs. $24.19 \pm 7.34$, Stress vs. Stress+Caff, \#\# $\mathrm{p}<0.01 ; \mathrm{n}=15)(\mathrm{A})$; and percentage change of control and stress cardiac myocytes after exposure to caffeine $(10 \mathrm{mM})\left(33.65 \pm 6.97\right.$ vs $56.74 \pm 5.11 \%$, Con vs. Stress, $\left.{ }^{* *} \mathrm{p}<0.01 ; \mathrm{n}=15\right)(B)$. $*^{* *} \mathrm{p}<0.01$ vs. Control; \#\#p<0.01 vs. Stress.
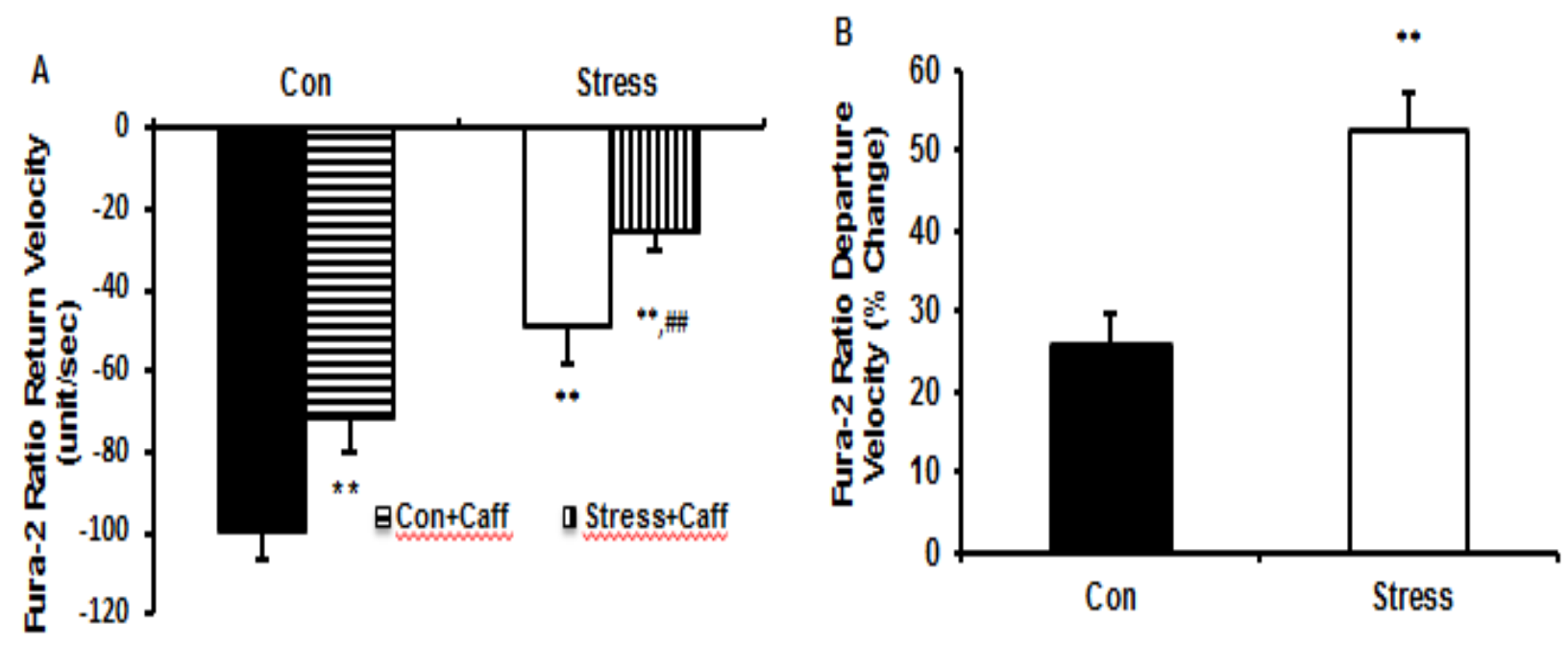

Figure 21: Bar graph depicting calcium return velocity in control and stress cardiac myocytes before and after exposure to caffeine $(10 \mathrm{mM})\left(-99.70 \pm 6.88\right.$ vs. $-49.07 \pm 8.07$, Con vs. Stress, ${ }^{* *} \mathrm{p}<0.01 ;-99.70 \pm 6.88$ vs. $-71.94 \pm 8.80$, Con vs. Con+Caff, $* * \mathrm{p}<0.01 ;-49.07 \pm 8.07$ vs. $-25.69 \pm 4.52$, Stress vs. Stress+Caff, \#\# $\mathrm{p}<0.01 ; \mathrm{n}=15)(\mathrm{A})$; and percentage change of control and stress cardiac myocytes after exposure to caffeine $(10 \mathrm{mM})\left(23.21 \pm 4.42 v s 50.88 \pm 6.92 \%\right.$, Con vs. Stress, $\left.{ }^{* *} \mathrm{p}<0.01 ; \mathrm{n}=15\right)(B) .{ }^{* *} \mathrm{p}<0.01$ vs. Control; \#\#p $<0.01$ vs. Stress. 

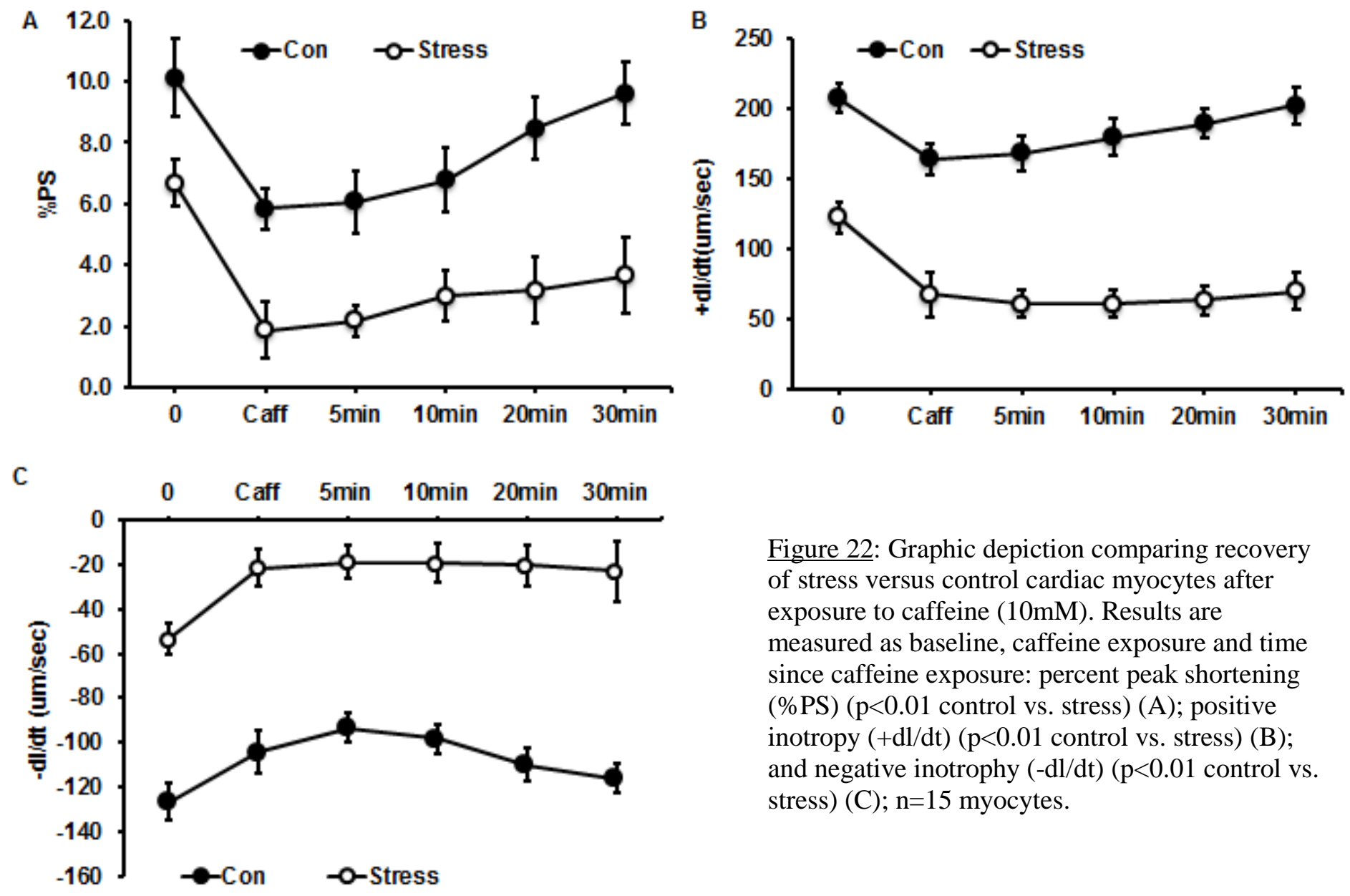

Figure 22: Graphic depiction comparing recovery of stress versus control cardiac myocytes after exposure to caffeine $(10 \mathrm{mM})$. Results are measured as baseline, caffeine exposure and time since caffeine exposure: percent peak shortening (\%PS) $(\mathrm{p}<0.01$ control vs. stress) (A); positive inotropy $(+\mathrm{dl} / \mathrm{dt})(\mathrm{p}<0.01$ control vs. stress) (B); and negative inotrophy $(-\mathrm{dl} / \mathrm{dt})(\mathrm{p}<0.01$ control vs. stress) (C); $\mathrm{n}=15$ myocytes. 

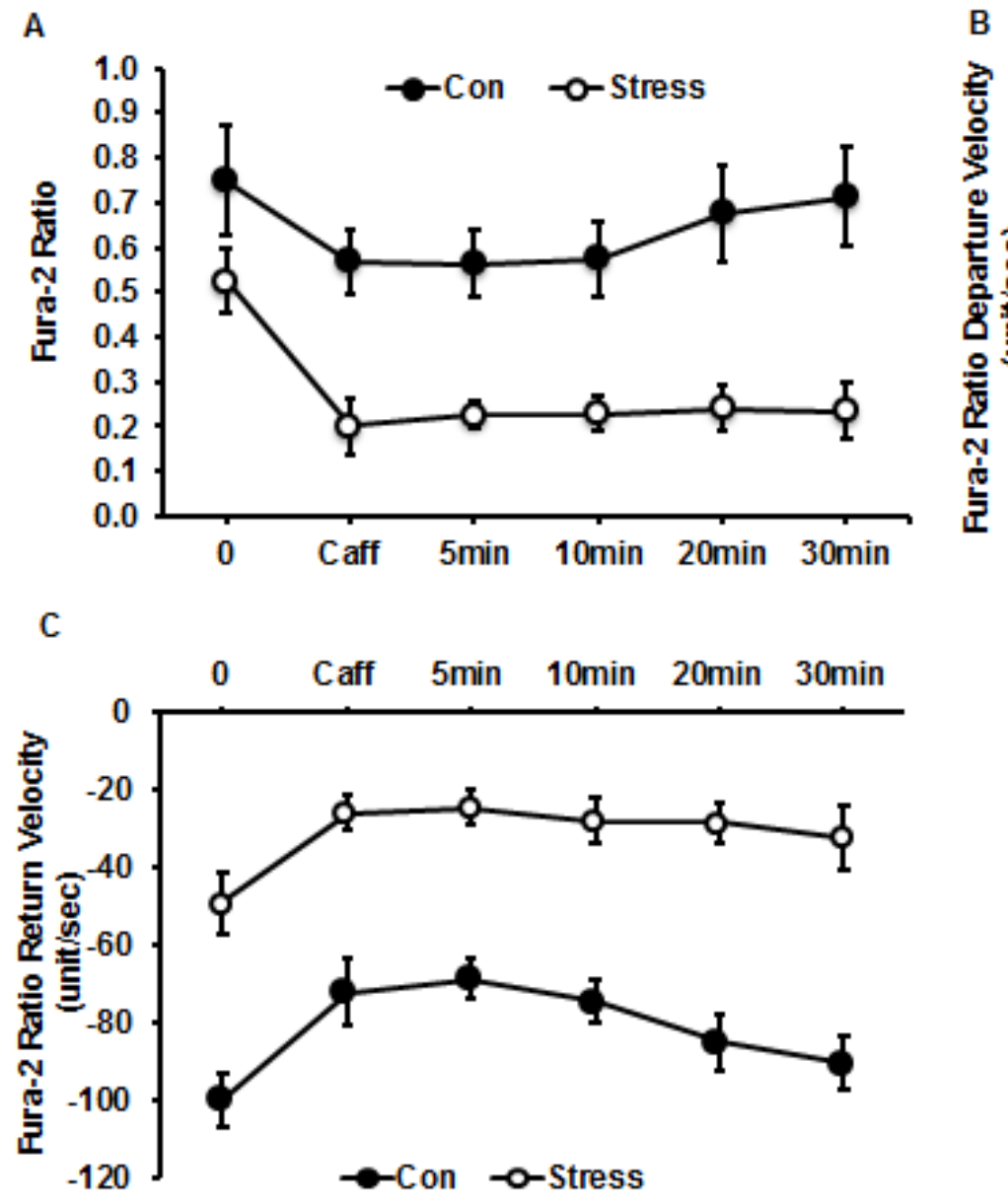

Figure 23: Graphic depiction comparing recovery of stress versus control cardiac myocytes after exposure to caffeine $(10 \mathrm{mM})$. Results are measured as baseline, caffeine exposure and time since caffeine exposure: change in $\mathrm{Ca}^{2+}$ concentration $(\mathrm{p}<0.05$ control vs. stress) (A); $\mathrm{Ca}^{2+}$ departure velocity ( $<<0.01$ control vs. stress) (B); and $\mathrm{Ca}^{2+}$ return velocity ( $p<0.01$ control vs. stress) $(C)$; $\mathrm{n}=15$ myocytes. 
A

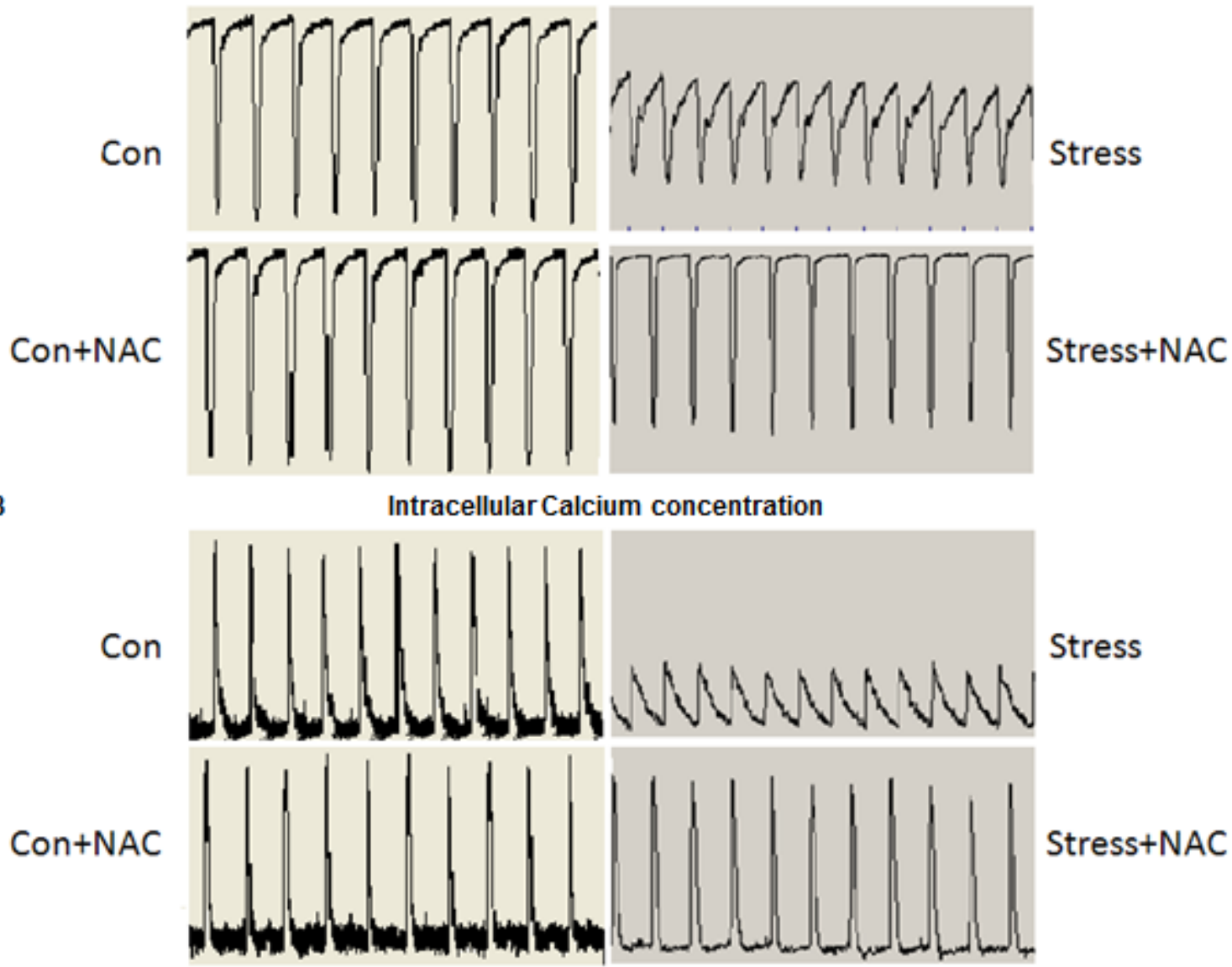

Figure 24: Tracing of control and stress cardiac myocytes contraction (A) and intracellular $\mathrm{Ca}^{2+}$ concentration (B) with 30 minutes of perifusion of KHB alone or with NAC $\left(10^{-3} \mathrm{M}\right)$ after exposure to caffeine $(10 \mathrm{mM})$. 

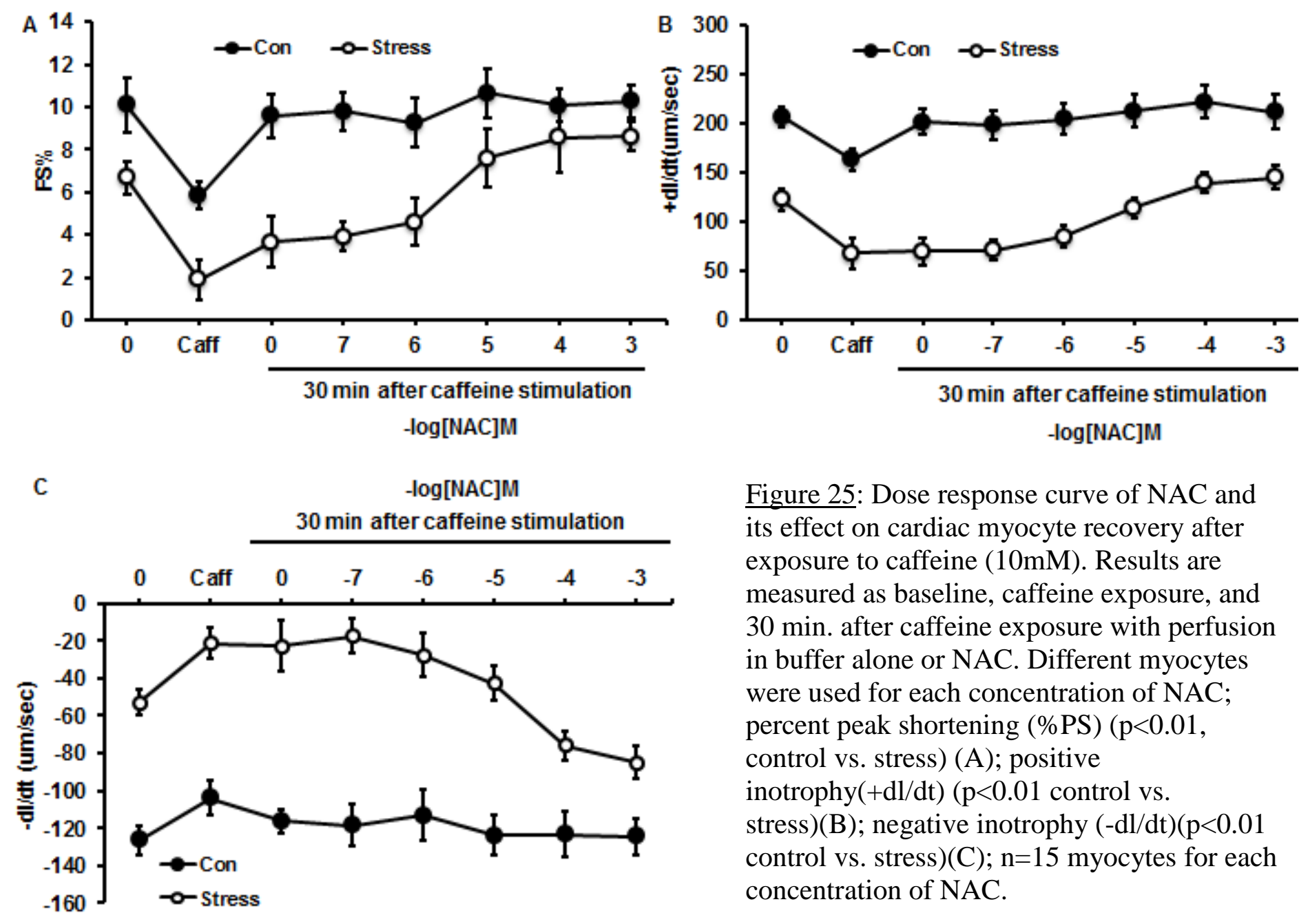

Figure 25: Dose response curve of NAC and its effect on cardiac myocyte recovery after exposure to caffeine $(10 \mathrm{mM})$. Results are measured as baseline, caffeine exposure, and 30 min. after caffeine exposure with perfusion in buffer alone or NAC. Different myocytes were used for each concentration of NAC; percent peak shortening (\%PS) $(\mathrm{p}<0.01$, control vs. stress) (A); positive inotrophy $(+\mathrm{dl} / \mathrm{dt})(\mathrm{p}<0.01$ control vs. stress)(B); negative inotrophy (-dl/dt) $(\mathrm{p}<0.01$ control vs. stress)(C); $n=15$ myocytes for each concentration of NAC. 


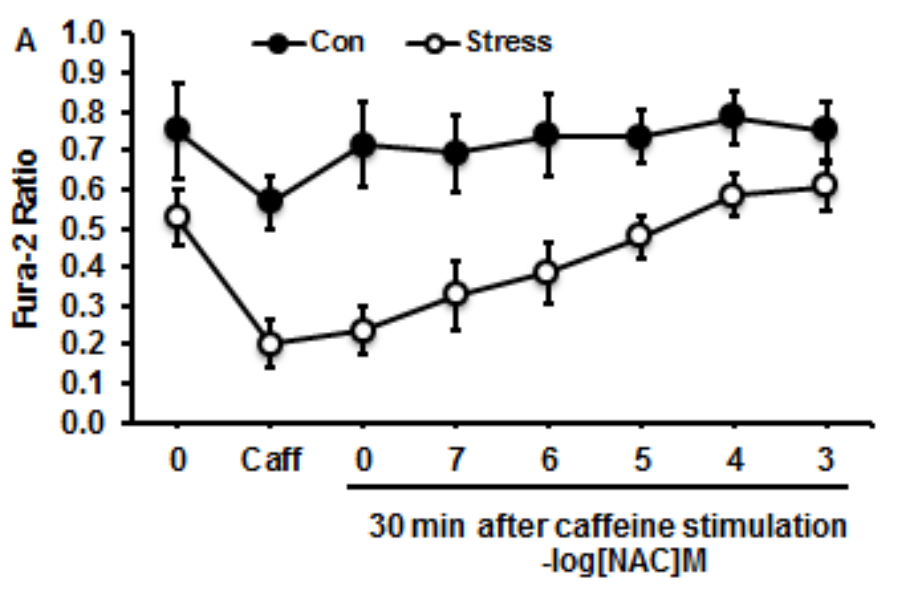

$-\log [\mathrm{NAC}] \mathrm{M}$

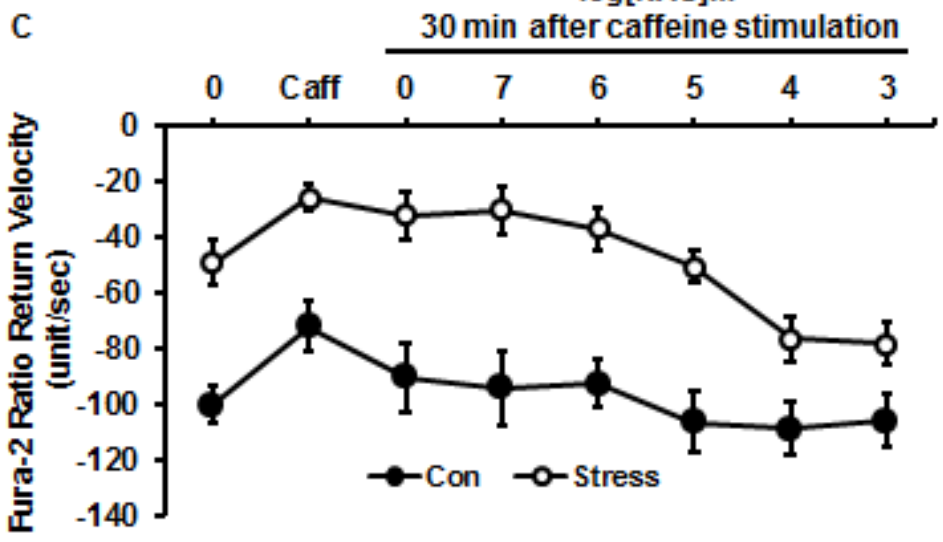

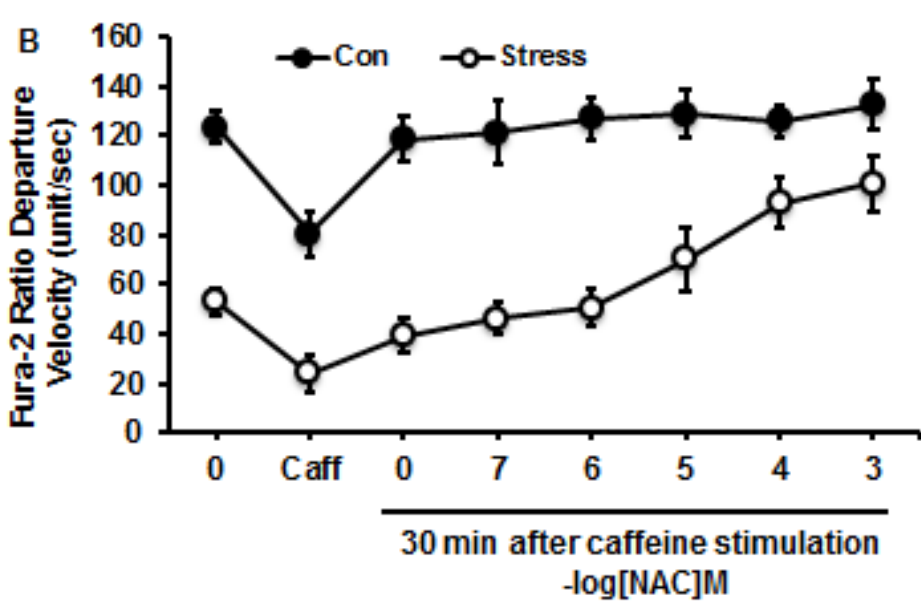

Figure 26: Dose response curve of NAC and its effect on cardiac myocyte recovery after exposure to caffeine $(10 \mathrm{mM})$. Results are measured as baseline, caffeine exposure, and 30 min. after caffeine exposure with perfusion in buffer alone or NAC. Different myocytes were used for each concentration of NAC; change in $\mathrm{Ca}^{2+}$ concentration $(\mathrm{p}<0.01$, control vs. stress) (A); $\mathrm{Ca}^{2+}$ departure velocity $(\mathrm{p}<0.01$ control vs. stress)(B); $\mathrm{Ca}^{2+}$ return velocity $(p<0.01$ control vs. stress)(C); $n=15$ myocytes 
A
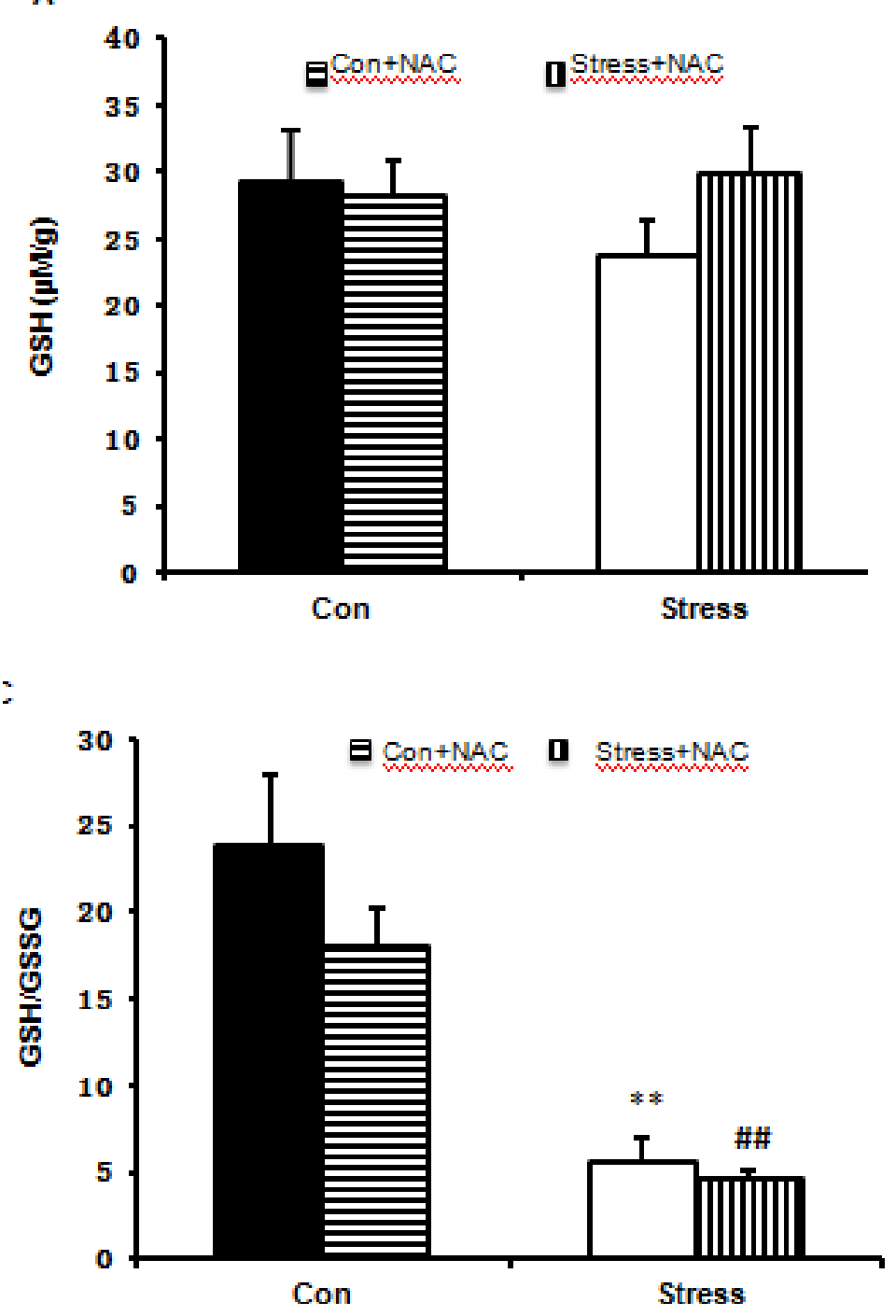

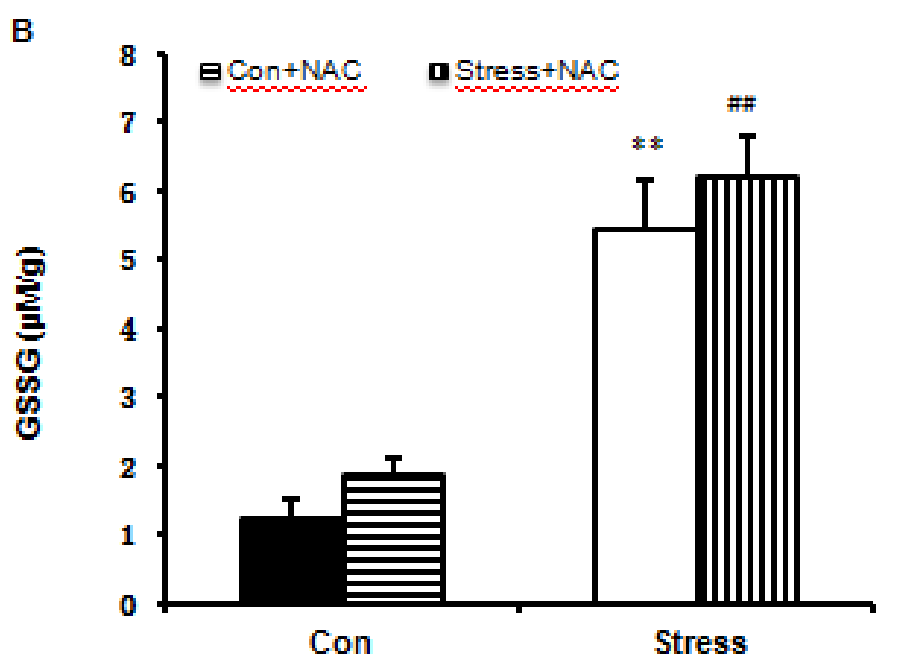

Figure 27: Bar graph depicting the effects of $10^{-3}$ NAC on GSH concentration (29.24 \pm 4.75 vs. $23.63 \pm 3.0$, control vs. stress; $28.29 \pm 4.07$ vs. $30.05 \pm 5.42$ control + NAC vs. stress +NAC p>0.05 $\mathrm{n}=15$ ) (A), GSSG concentration (1.23 \pm 0.20 vs. $5.46 \pm 0.71$, control vs. stress ** $\mathrm{p}<0.01 ; 1.87 \pm 0.44$ vs. $6.20 \pm 0.94$ control + NAC vs. stress + NAC, $\# \# \mathrm{p}<0.01 \mathrm{n}=15)(B)$, and on the GSH/GSSG ratio (23.76 \pm 4.20 vs. $17.93 \pm 2.30 \mu \mathrm{M} / \mathrm{mg}$, Control vs. Control +NAC; $5.58 \pm 1.42$ vs. $4.66 \pm 0.52$ Stress vs. Stress + NAC, $\mathrm{p}=\mathrm{NS}$; Control vs. Stress $* * \mathrm{p}<0.01$; Control + NAC vs. Stress + NAC, \#\# $\mathrm{p}<0.01 \mathrm{n}=10$ ) (C). 


\section{Discussion}

A compelling body of basic and clinical literature supports a pathogenic role for oxidative stress in animal models and human beings with cardiomyopathies and heart failure (Akki et al; Kuroda et al, 2010). Oxidative stress is said to occur when there is an imbalance between the production of reactive oxygen species (ROS) and the anti-oxidant capacity of the cell. Oxidative stress is increased in failing myocardium and studies have implicated redoxmediated mechanisms by ROS in several aspects of myocardial failure (Sawyer et al, 2002). Prolonged adrenergic stimulation can cause myocardial and systemic oxidative stress, with an increase in ROS without appropriate increase in antioxidants. Mitochondria are the major source of ROS due to electron leakage from the electron transport chain to react with oxygen and form superoxide (Sawyer et al, 2002). Indeed, prolonged $\beta_{2}$-adrenoreceptor activation has been reported to lead to activation of NADPH oxidase, the production of ROS, and p38 MAPK activation. Treatment with n-acetylcysteine (NAC) prevented p38 MAPK activation, indicating that p38 MAPK is a downstream target of ROS (Xu et al, 2011). Besides NADPH oxidase, cytochrome P450 and xanthine oxidase are sources of ROS. Antioxidant enzyme systems are present to prevent oxidative damage and include superoxide dismutase, catalase and glutathione peroxidase. A delicate balance exists between the production of intracellular oxidants and antioxidants to maintain cellular homeostasis. Enhanced oxidation and/or inadequate reducing capacity result in oxidative stress from uncontrolled free radical activation. Such free radical activation in the heart can cause myocardial contractile dysfunction (Akki et al, 2009; Goldhaber and Qayyum, 2000; Zima and Blatter, 2006). Stress offspring show signs of myocardial dysfunction and oxidative stress with reduced ATP levels (Fig.8) and GSH/GSSG (Fig. 9C), respectively 
Both in vivo and in vitro administration of n-acetylcysteine (NAC) has been shown to raise intracellular concentrations of reduced glutathione (GSH) by providing an additional source of cysteine as a precursor (Choy et al, 2010; Han et al, 2010; Kerksich and Willoughby, 2005). Glutathione is a tripeptide consisting of glutamate, cysteine, and glycine, with cysteine being the limiting amino acid in the production of glutathione. Glutathione is the main cytosolic redox buffer in cardiomyocytes with the ratio of reduced glutathione to glutathione disulfide (oxidized form)(GSH/GSSG) being controlled by the pentose phosphate pathway by providing nicotinamide-adenine dinucleotide phosphate (NADPH) which is needed by glutathione reductase to covert GSSG into GSH (Zima and Blatter, 2006; Sawyer et al, 2002). Because GSH can directly scavenge free radicals including ROS or indirectly through glutathione peroxidase, a high cytosolic GSH/GSSG ratio is critical for antioxidant defense (Zima and Blatter, 2006).

Besides being a GSH precursor (Zafarullah et al, 2003), NAC can reduce disulfide bonds (Harada et al, 2004), and scavenge free radicals (Kelly, 1998). Glutathione deficiency occurs in many diseases due to factors including viral protein-mediated GSH depletion, uncontrolled inflammatory reactions, and increased generation of free radicals. Clinically, NAC is used as a mucolytic agent, for acetaminophen overdose, and in disease states characterized by low cysteine and GSH concentrations like HIV, heart disease, cancer, and pulmonary diseases (Kelly, 1998; Atkuri et al, 2007; Zafarullah et al, 2003). The doses of NAC used in humans have typically ranged from 200-1,000 mgs orally daily (Borges-Santos et al, 2012; De Rosa et al, 2000). These doses have been demonstrated to significantly increase plasma cysteine and GSH, each by approximately 100 uM (Borges-Santos et al, 2012; De Rosa et al, 2000). Oral NAC doses of 200-400 mg also have been reported to achieve plasma concentrations of 0.35-4 mg/L (BorgesSantos et al, 2012; De Rosa et al, 2000). Accordingly, we used the concentration range of $10^{-3}$ to 
$10^{-7} \mathrm{M}$ NAC for in vitro experiments based on a molecular weight of 163 and published pharmacokinetic data in human beings (Holdiness,1991).

Heart failure is characterized by myocardial remodeling, left ventricular dysfunction, and impaired myocyte calcium handling (Dash et al, 2001; Hasenfuss \& Pieske, 2002; Luo et al, 2006). Contraction and force generation in muscle is regulated by cytosolic concentrations of calcium $\left(\mathrm{Ca}^{2+}\right)$, which is stored in the sacroplasmic reticulum (SR) and is available for immediate release into the cytosol. Changes in intracellular $\mathrm{Ca}^{2+}$ handling are associated with myocardial dysfunction presenting with reduced $\mathrm{Ca}^{2+}$ transient amplitude, increased $\mathrm{Ca}^{2+}$ transient duration, prolonged $\mathrm{Ca}^{2+}$ transient decay time, and decreased SR $\mathrm{Ca}^{2+}$ load (Hobai and O’Rourke, 2001; Hasenfuss \& Pieske, 2002; Houser and Margulies, 2003; Belevych et al, 2007; Kuster et al, 2010). It is clear that altered myocyte $\mathrm{Ca}^{2+}$ handling is involved in our Stress model of reversible myocardial dysfunction (Figs.13, 14, 22, 23, 25, 26).

Cardiac contraction consists of an electrical excitation phase followed by a contractile phase (E-C coupling). Depolarization of the sinoatrial node initiates the electrical phase causing a wave of depolarization to spread via the conduction system through the atria and ventricles. The current flows from a depolarized cardiomyocyte to its resting neighbor through gap junctions. The depolarization of the myocyte allows a small amount of $\mathrm{Ca}^{2+}$ to cross the sarcolemmal L-type calcium channel (LTCC), where it activates the Ryanodine receptor (RyR2) to release a larger amount of $\mathrm{Ca}^{2+}$ into the cytoplasm from the SR. The cytoplasmic $\mathrm{Ca}^{2+}$ then binds to Troponin C, enabling actin-myosin binding and sliding of the myofilaments and resulting in sarcomere shortening and myocardial contraction. Calcium is returned to the SR through the sacro-endoplasmic reticulum calcium ATPase (SERCA2), which re-sequesters $\mathrm{Ca}^{2+}$ back into the SR at the expense of ATP. $\mathrm{Ca}^{2+}$ also is removed from the cell through the 
sacrolemmal sodium-calcium exchanger (NCX) and plasmalemmal $\mathrm{Ca}^{2+}$ ATPase to balance the $\mathrm{Ca}^{2+}$ that entered through the LTCC (Kushnir \& Marks, 2010; Zalk et al, 2007; Belevych et al, 2007; Hasenfuss and Pieske, 2002) (Fig. 28). Therefore, expression and activity of the receptors/transporters of $\mathrm{Ca}^{2+}$ release and uptake are related to the force of myocardial systolic contraction and diastolic relaxation.

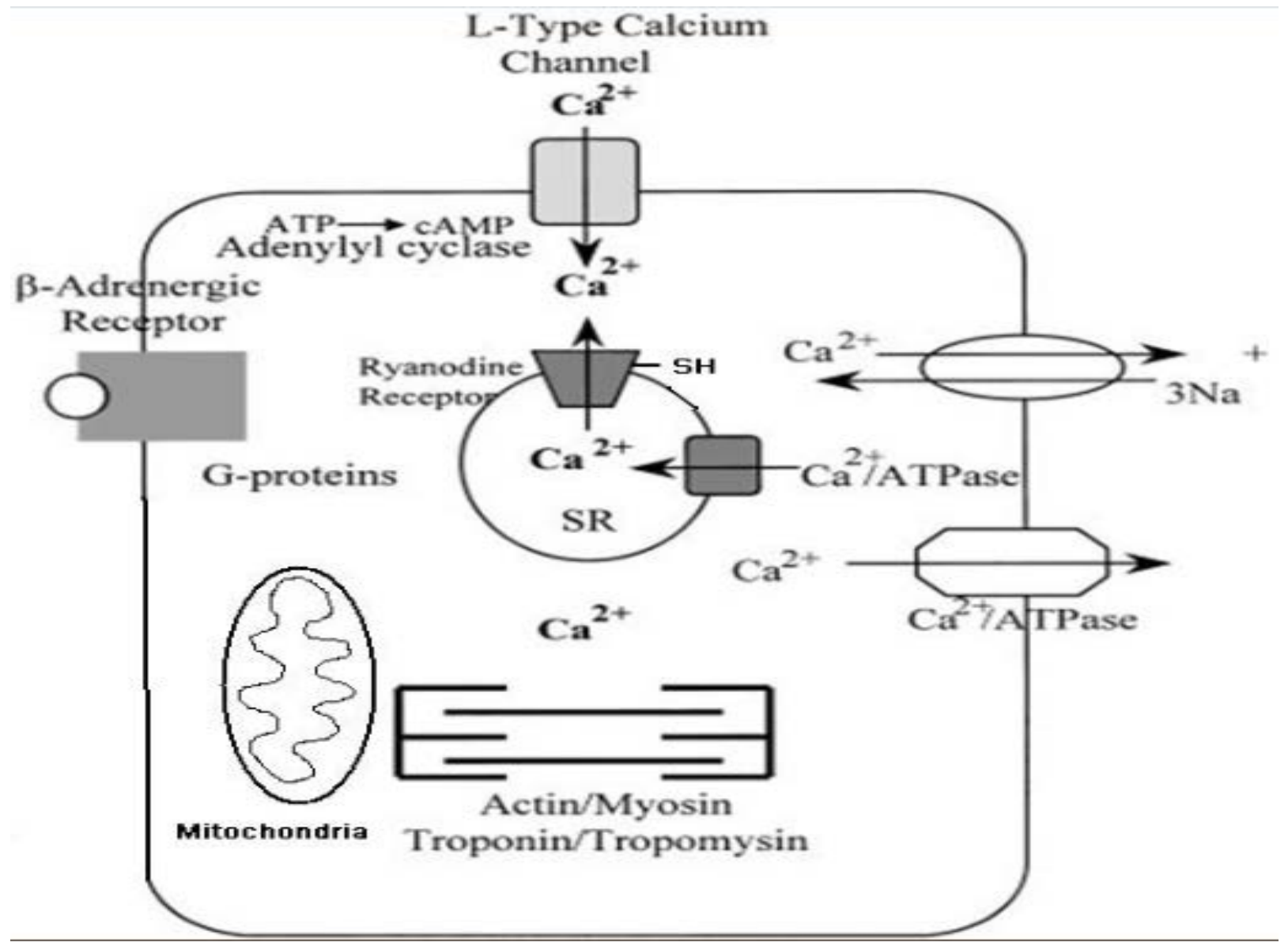

Figure 28: Schematic diagram illustrating the movement of $\mathrm{Ca}^{2+}$ into the myocyte to cause intracellular $\mathrm{Ca}^{2+}$ release followed by resequestration of $\mathrm{Ca}^{2+}$ back into the sarcoplasmic reticulum (SR) and extrusion back into the extracellular space. A small amount of $\mathrm{Ca}^{2+}$ enters the myocyte through L-type calcium channels (LTCC) which triggers a large release of Ca ${ }^{2+}$ from the SR through activation of the Ryanodine receptor (RyR2). $\mathrm{Ca}^{2+}$ is resequestered into the SR by the sacroendoplasmic reticulum calcium ATPase ( $\mathrm{Ca}^{2+}$ /ATPase; SERCA2). $\mathrm{Ca}^{2+}$ is extruded from the cell largely through the sodium/calcium exchanger (NCX) and the plasmalemmal calcium ATPase. Contraction can be modulated through the Gprotein coupled beta adrenergic receptor. Binding to the beta-receptor will lead to adenylyl cyclase activation, cyclic AMP production and PKA activation. The targets of PKA include the LTCC, RYR2, and phospholamban (regulatory protein of SERCA2). Therefore, beta-receptor activation typically leads to enhanced contraction and relaxation of the myocyte. (Modified from Kan and Finkel, 2003) 
Two possibilities may explain the altered $\mathrm{Ca}^{2+}$ handling in our model. First, there could be decreased reuptake of $\mathrm{Ca}^{2+}$ into the SR due to decreased activity of SERCA2 (due to redox mechanisms of phosphorylation status of phospholamban) with possible increase in activity of the NCX (increased extrusion of $\mathrm{Ca}^{2+}$ out of the cell helps maintain normal diastolic $\mathrm{Ca}^{2+}$ but decreases the amount available for SR reuptake). The second possibility is $\mathrm{Ca}^{2+}$ leaking out of the SR during diastole due to dysfunctional activity of RYR2 (Marx et al, 2000; Hobai and O’Rourke, 2001; Inesi et al, 2008; Houser and Margulies, 2003; Belevych et al, 2007; Satoh et al, 2000; Kuster et al, 2010; Luo et al, 2006;Zima et al, 2010). Altered function of these transporters could explain the reduced percent peak shortening (\% PS), positive inotrophy $(+\mathrm{dl} / \mathrm{dt})$, negative inotrophy (-dl/dt), $\mathrm{Ca}^{2+}$ release, departure velocity, and return velocity upon stimulation with isoproterenol (Figs. 11, 12), extracellular calcium (Figs. 13, 14), and caffeine (Figs 16-21) in Stress myocytes.

Prenatally stressed (PS) offspring have prolonged, exaggerated responses when presented with an acute stressor as adults due to impaired negative feedback of their HPA axis (Braastad, 1998; Maccari and Fletcher, 2007; Mastorci et al, 2009). This PS model has been shown to have increased concentrations of corticotropin-releasing factor (CRF) (Cratty et al, 1995) and corticosterone (Ward et al, 1999).We now show that this lengthened stress response leads to an increase in catecholamines in Stress offspring after behavioral stress, as seen by elevated epinephrine and norepinephrine the day after their second restraint (Fig 7). This is important because prolonged beta-adrenergic receptor activity is known to result in cardiac hypertrophy, depressed left ventricular function and premature mortality (Dash et al, 2001).

Catecholamines activate $\mathrm{G}_{\mathrm{s}}$-protein coupled receptors through $\beta$ adrenergic receptors leading to PKA activation (Rapacciuolo et al, 2001) and increased cardiac contractility (Zheng, 
et al, 2000). Activated PKA will phosphorylate RyR2, activating the $\mathrm{Ca}^{2+}$ release channel partly by increasing the sensitivity of RyR2 to cytosolic $\mathrm{Ca}^{2+}$ (Marx et al, 2000). Prolonged catecholamine signaling, however, is detrimental, leading to increased activation of PKA and hyperphosphorylation of the RyR2 receptor, $\mathrm{Ca}^{2+}$ leak out of the SR and contractile dysfunction. This may be due to the dissociation of calstabin2 (a RyR2 regulatory protein), from RyR2 upon PKA phosphorylation resulting in increased sensitivity of RyR2 to $\mathrm{Ca}^{2+}$ induced activation. Prolonged increased RyR2 sensitivity to cytosolic $\mathrm{Ca}^{2+}$ leads to inappropriate $\mathrm{Ca}^{2+}$ release during diastole ( $\mathrm{Ca}^{2+}$ leak) and reduces SR Ca ${ }^{2+}$ stores (Marx et al,2000; Bellinger et al, 2008). Hyperphosphorylation of RyR2 and the dissociation of calstabin2 uncouples multiple RyRs and disturbs coupled gating. Coupled gating is important for enabling the opening and closing of RyRs in a given SR T-tubule junction in a coordinated manner. Uncoupled channels will result in some channels not closing during diastole and $\mathrm{Ca}^{2+}$ leak (Hasenfuss and Pieske, 2002; Marx et al, 2001). Reduced phosphatase association with RyR2 could play a role in $\mathrm{Ca}^{2+}$ leak with decreased inactivation of RyR2 (Marx et al, 2000). Stress animals had reduced concentrations of ATP (Fig. 8). This could be due to increased SERCA2 activity in an effort to compensate for $\mathrm{Ca}^{2+}$ leak through RyR2 (Hasenfuss and Pieske, 2002; Bellinger et al, 2008; Zima et al, 2010). There is evidence that redox mechanisms can affect the activity of several $\mathrm{Ca}^{2+}$ transporters. Salama first described sulfhydryl regulation of the RyR2, where it was seen that oxidation via cysteine caused formation of disulfide bonds and opening of the channel and SR $\mathrm{Ca}^{2+}$ release. Conversely, addition of sulfhydryl reducing agents like reduced glutathione (GSH) reduced the disulphide bonds, regenerating the free SH groups on SR proteins, closing the channel and activating re-uptake of $\mathrm{Ca}^{2+}$ (Salama et al, 1992; Trimm et al, 1986; Györke and Carnes, 2008; Zima and Blatter, 2006).Prolonged adrenergic stimulation has been suggested to 
cause myocardial and systemic oxidative stress (Sawyer et al, 2002). Reactive oxygen species (ROS) are capable of reacting with cysteines causing a conformational change in RyR2 and $\mathrm{Ca}^{2+}$ leak (Terentyev et al, 2008; Belevych et al, 2007; Györke and Carnes, 2008; Zima and Blatter, 2006). SERCA2 may decrease in activity as a result of oxidation by ROS (Scherer and Deamer, 1986; Lancel et al, 2010; Kuster et al, 2010; Zima and Blatter, 2006) while the NCX can be activated by ROS (Reeves et al, 1986; Goldhaber and Qayyum, 2000; Kuster et al, 2010; Zima and Blatter, 2006). In addition, p38MAPK is redox-sensitive and is activated by ROS (Saitoh et al, 2007; Filomeni et al, 2003; Veal et al, 2004). Stress offspring have evidence of mitochondrial dysfunction and oxidative stress seen by reduced ATP (Fig.8), and GSH/GSSG (Fig. 9C), respectively. Studies have shown that treatment of control myocytes with an oxidizing agent weakens the amplitude of $\mathrm{Ca}^{2+}$ transients while treating heart failure myocytes with an antioxidant increases the amplitude of the $\mathrm{Ca}^{2+}$ transient up to control values. Treatment of control myocytes with an antioxidant, however, had no effect. These results are consistent with the idea that reversible oxidation of components of the E-C coupling machinery is minimal in normal myocytes (Terentyev et al, 2008). This concept agrees with the present results as well; NAC was able to reverse dysfunction in Stress myocytes but had no effect on Control myocytes (Figs. 10, 11A-C, 12A-C, 13A-C, 14A-C, 24A\&B, 25A-C, 26A-C).

There is a blunted response to the $\beta$-adrenergic agonist, isoproterenol, in the Stress model (Kan et al, 2005; Chen et al, 2009; Figs. 11A-C; 12A-C). This may be because RyR2 is already PKA hyperphosphorylated and further phosphorylation (through $\beta$-adrenergic receptors/ $\mathrm{G}_{\mathrm{s}}$ pathway) cannot occur (Marx et al, 2000). Phosphorylation of RyR2 increases the probability that it will be in the open state; hyperphosphorlyation can result in incomplete channel closure and allows $\mathrm{Ca}^{2+}$ to leak out of the SR. Therefore, upon stimulation, these myocytes have a lower 
concentration of $\mathrm{Ca}^{2+}$ available for release from the SR compared to normal myocytes (Zalk et al, 2007; Belevych et al, 2007). Stress myocytes had reduced SR Ca ${ }^{2+}$ release when stimulated with isoproterenol or calcium (Figs. $12 \mathrm{~A} ; 14 \mathrm{~A})$, NAC $\left(10^{-3}\right)$ reversed this defect in the Stress myocytes without affecting the Control myocytes (Figs. $12 \mathrm{~A} ; 14 \mathrm{~A})$. NAC $\left(10^{-3}\right)$ also reverses the positive (+dl/dt) and negative (-dl/dt) inotropic dysfunction in response to isoproterenol and extracellular calcium (Fig. 11 A-C, 13A-C) in the Stress myocytes without affecting the Control myocytes. This may occur through redox mechanisms of NAC on RyR2, closing the channel and stopping the $\mathrm{Ca}^{2+}$ leak.

Catecholamine signaling pathways have been shown to activate mitogen-activated protein (MAP) kinases, including stress-activated p38 MAPK (Rapacciuolo et al, 2001; Dash et al, 2003; Xu et al, 2011) with the stimulatory effects of the $\beta 2$-adrenergic receptor on p38 MAPK being mediated by a PKA-dependent pathway (Zheng et al, 2000). Upon activation, p38 MAPK then provides a negative feedback to PKA-mediated positive contractile response (Zheng et al, 2000). We have previously reported in Stress offspring elevated phosphorylated p38 MAPK and reversibility of myocardial dysfunction with the p38 MAP kinase inhibitor SB-203580 (Kan et al, 2005; Chen et al, 2009). Activated p38 will phosphorylate an array of intracellular targets including numerous transcription factors, resulting in reprogramming of gene expression (Liang \& Molkentin, 2003) and cardiac remodeling (Dash et al, 2003). The p38 MAPK pathway can stimulate hypertrophic growth (Zechner et al, 1997; Wenzel et al, 2005), be pro-apoptotic (Wang et al, 1998), or anti-apoptotic (Zechner et al, 1998), indicating a significant role for p38 signaling in cardiac pathologies. Authors of in vivo and in vitro studies have reported that cardiac specific activation of p38 MAPK markedly attenuates cardiac contractility (Liao et al, 2001; Liao et al, 2002). Increased activation of p38 during prolonged episodes of stress may counterbalance the 
PKA-mediated positive contractile response by decreasing the myofilament responsiveness to calcium (Liao et al, 2002). Cardiac stress through activation of p38 MAPK may lead to downregulation of SERCA2 (Andrews et al, 2003).This will result in increased diastolic intracellular calcium concentration (Andrews et al, 2003). SERCA2 is regulated by phospholamban. The dephosphorylated form inhibits SERCA2. Phosphorylation of phospholamban by PKA stimulates SERCA2 activity (Hasenfuss and Pieske, 2002; Dash et al, 2001; Marx et al, 2000). If p38 MAPK feeds back to negatively regulate PKA, decreased phosphorylation of phospholamban could decrease SERCA2 activity and SR $\mathrm{Ca}^{2+}$ reuptake. Exposure of myocytes to caffeine sensitizes RyR2 to activation by $\mathrm{Ca}^{2+}$ and leads to a massive release of $\mathrm{Ca}^{2+}$ from the SR. Caffeine potentiates $\mathrm{SR} \mathrm{Ca}^{2+}$ release and myocyte contraction only transiently because it depletes the SR of $\mathrm{Ca}^{2+}$ (Zalk et al, 2007). After caffeine exposure, the amplitude of further contractions and $\mathrm{SR} \mathrm{Ca}^{2+}$ release will be reduced until the $\mathrm{Ca}^{2+}$ is returned to the SR through SERCA2. This pattern was seen in myocytes exposed to caffeine. Caffeine exposure resulted in a spike in contraction and intracellular $\mathrm{Ca}^{2+}$ followed by a reduction in amplitude of both (Fig. $15 \mathrm{~A} \& \mathrm{~B}$ ) with return to baseline in Control myocytes within 30 minutes (Fig. 24 A\&B). Stress myocytes showed an initial increase followed by a decrease in amplitude of contraction and intracellular $\mathrm{Ca}^{2+}$ (Fig. $15 \mathrm{~A} \& \mathrm{~B}$ ) but were unable to return to baseline within 30 minutes (Fig. 24 A\&B). Perifusion of the myocytes with NAC $\left(10^{-3}\right)$ after caffeine exposure allowed the Stress myocytes to return to baseline (Fig. 24 A\&B) while having no effect on the Control myocytes, which were able to return to baseline without NAC (Figs. 24 A\&B;). This indicates that the Stress myocytes were not effectively taking $\mathrm{Ca}^{2+}$ back up into the SR, which would point to decreased SERCA2 activity. However, $\mathrm{Ca}^{2+}$ leak through RyR2 also could play a role. 
The initial exposure to caffeine caused an increase in contraction and intracellular $\mathrm{Ca}^{2+}$, followed by a decrease in percent peak shortening (Fig. 16 A), positive inotrophy (+dl/dt) (Fig. 17A), negative inotrophy (-dl/dt) (Fig.18A), SR Ca ${ }^{2+}$ release (Fig. 19A), SR $\mathrm{Ca}^{2+}$ departure velocity (Fig. 20A), and SR $\mathrm{Ca}^{2+}$ return velocity (Fig 21A). Baseline values of these 6 variables were reduced in Stress myocytes compared to Control. The decrease in these variables occurred in both Control and Stress myocytes. However, the decrease was significantly more pronounced $(\mathrm{p}<0.01)$ in the Stress myocytes (Figs. 16B, 17B, 18B, 19B, 20B, 21B). The Control myocytes returned to baseline values of all 6 of these variables within 30 minutes (Figs. 22A-C; 23A-C). The Stress myocytes were not able to return to baseline (Figs. 22A-C; 23A-C). Myocytes were perifused with NAC after exposure to caffeine. Stress myocytes perifused with the higher concentrations of NAC $\left(10^{-5}, 10^{-4}, 10^{-3}\right)$ approached baseline values of percent peak shortening (Fig. 25 A), positive inotrophy (+dl/dt) (Fig. 25B), negative inotrophy (-dl/dt) (Fig.25C), SR $\mathrm{Ca}^{2+}$ release (Fig. 26A), SR Ca ${ }^{2+}$ departure velocity (Fig. 26B), and SR $\mathrm{Ca}^{2+}$ return velocity (Fig 26C). NAC had no effect on Control myocytes which were able to return to baseline spontaneously (Figs. 25 A-C; 26 A-C). Perifusion with the higher concentrations of NAC (10 ${ }^{5}, 10^{-4}, 10^{-3}$ ) not only allowed Stress myocytes to return to Stress baseline values but surpassed them and approached baseline for the Control myocytes (Figs. 25 A-C; 26 A-C). Again, NAC may be working through redox mechanisms on the $\mathrm{Ca}^{2+}$ channels of the Stress myocytes without affecting the Control myocytes.

In summary, changes in myocyte $\mathrm{Ca}^{2+}$ handling could be attributed partially to redox modifications and/or phosphorylation of $\mathrm{Ca}^{2+}$ transporters and/or their regulatory proteins. Chronically elevated circulating catecholamines can modify these transporters/proteins by phosphorylation through PKA (Rapacciuolo et al, 2001; Zheng et al, 2000) or by redox 
mechanisms through oxidative stress (Terentyev et al, 2008; Neri et al, 2007; Osadchii, 2007) in the prenatal stress model. NAC, possibly through redox mechanisms, can reverse the myocardial dysfunction seen in this model after behavioral stress.

While NAC was able to reverse the dysfunction in Stress myocytes in response to isoproterenol, extracellular calcium, and caffeine, NAC failed to normalize both GSSG and GSH/GSSG levels in Stress myocytes (Fig.27). These data indicate that NAC mediates its inotropic effects independent of increasing GSH and mostly functions through redox mechanisms in the prenatal stress model. Indeed, reversal of both positive and negative inotrophy by NAC is consistent with sulfhydryl regulation of RyR2 or SERCA-2. Considerably more detailed physiologic and biochemical studies will be necessary before drawing any definitive conclusions regarding which transporters that are affected by NAC in this model.

In conclusion, we have provided compelling evidence of reversal of cardiac myocyte dysfunction by NAC in a behaviorally stressed cardiomyopathy model. Potential clinical implications of these data include the possibility that cardiomyopathies may respond to NAC therapy. NAC therapy is already being explored for treating HIV infection (Roederer et al, 1992).These studies are being conducted based on data indicating enhanced oxidative stress and decreased glutathione concentrations following HIV infection (Nakamura et al,2002). In fact, Chen et al (2012) reported reversal of myocardial dysfunction with NAC in a Tat (HIV protein) transgenic murine model with increased oxidative stress. Further studies are needed to determine the exact role that NAC is playing and what transporters are being altered in this unique animal model of behavioral stress induced reversible cardiomyopathy. 


\section{Literature Cited}

Adolphs, R., D. Tranel, H. Damasio \& A. R. Damasio (1995) Fear and the human amygdala. J Neurosci, 15, 5879-91.

Ahlbom, E., V. Gogvadze, M. Chen, G. Celsi \& S. Ceccatelli (2000) Prenatal exposure to high levels of glucocorticoids increases the susceptibility of cerebellar granule cells to oxidative stress-induced cell death. Proc Natl Acad Sci U S A, 97, 14726-30.

Akashi, Y. J., D. S. Goldstein, G. Barbaro \& T. Ueyama (2008) Takotsubo cardiomyopathy: a new form of acute, reversible heart failure. Circulation, 118, 2754-62.

Akki, A., M. Zhang, C. Murdoch, A. Brewer \& A. M. Shah (2009) NADPH oxidase signaling and cardiac myocyte function. J Mol Cell Cardiol, 47, 15-22.

Anda, R., D. Williamson, D. Jones, C. Macera, E. Eaker, A. Glassman \& J. Marks (1993) Depressed affect, hopelessness, and the risk of ischemic heart disease in a cohort of U.S. adults. Epidemiology, 4, 285-94.

Andrews, C., P. D. Ho, W. H. Dillmann, C. C. Glembotski \& P. M. McDonough (2003) The MKK6-p38 MAPK pathway prolongs the cardiac contractile calcium transient, downregulates SERCA2, and activates NF-AT. Cardiovasc Res, 59, 46-56.

Atkuri, K. R., J. J. Mantovani \& L. A. Herzenberg (2007) N-Acetylcysteine--a safe antidote for cysteine/glutathione deficiency. Curr Opin Pharmacol, 7, 355-9.

Barbazanges, A., P. V. Piazza, M. Le Moal \& S. Maccari (1996) Maternal glucocorticoid secretion mediates long-term effects of prenatal stress. J Neurosci, 16, 3943-9.

Barefoot, J. C., M. J. Helms, D. B. Mark, J. A. Blumenthal, R. M. Califf, T. L. Haney, C. M. O'Connor, I. C. Siegler \& R. B. Williams (1996) Depression and long-term mortality risk in patients with coronary artery disease. Am J Cardiol, 78, 613-7.

Belevych, A., Z. Kubalova, D. Terentyev, R. L. Hamlin, C. A. Carnes \& S. Györke (2007) Enhanced ryanodine receptor-mediated calcium leak determines reduced sarcoplasmic reticulum calcium content in chronic canine heart failure. Biophys J, 93, 4083-92.

Bellinger, A. M., M. Mongillo \& A. R. Marks (2008) Stressed out: the skeletal muscle ryanodine receptor as a target of stress. $J$ Clin Invest, 118, 445-53.

Black, D. W. (1998) lowa record-linkage study: death rates in psychiatric patients. J Affect Disord, 50, 277-82.

Blumenthal, J. A., R. S. Williams, A. G. Wallace, R. B. Williams \& T. L. Needles (1982) Physiological and psychological variables predict compliance to prescribed exercise therapy in patients recovering from myocardial infarction. Psychosom Med, 44, 519-27.

Borges-Santos, M. D., F. Moreto, P. C. Pereira, Y. Ming-Yu \& R. C. Burini (2012) Plasma glutathione of $H I V(+)$ patients responded positively and differently to dietary supplementation with cysteine or glutamine. Nutrition.

Bouayed, J., H. Rammal, C. Younos \& R. Soulimani (2007) Positive correlation between peripheral blood granulocyte oxidative status and level of anxiety in mice. Eur J Pharmacol, 564, 146-9.

Braastad, B.O. 1998. Effects of prenatal stress on behaviour of offspring of laboratory and farmed mammals. Applied Animal Behaviour Science. 61:159-180.

Bruchas, M. R., B. B. Land, M. Aita, M. Xu, S. K. Barot, S. Li \& C. Chavkin (2007) Stress-induced p38 mitogen-activated protein kinase activation mediates kappa-opioid-dependent dysphoria. $J$ Neurosci, 27, 11614-23.

Brunton, P. J. (2010) Resetting the dynamic range of hypothalamic-pituitary-adrenal axis stress responses through pregnancy. J Neuroendocrinol, 22, 1198-213. 
Brunton, P. J. \& J. A. Russell (2010) Prenatal social stress in the rat programmes neuroendocrine and behavioural responses to stress in the adult offspring: sex-specific effects. J Neuroendocrinol, 22, 258-71.

Bybee, K. A. \& A. Prasad (2008) Stress-related cardiomyopathy syndromes. Circulation, 118, 397-409.

Carney, R. M., K. E. Freedland, S. A. Eisen, M. W. Rich \& A. S. Jaffe (1995) Major depression and medication adherence in elderly patients with coronary artery disease. Health Psychol, 14, 8890.

Carney, R. M., K. E. Freedland, M. W. Rich, L. J. Smith \& A. S. Jaffe (1993) Ventricular tachycardia and psychiatric depression in patients with coronary artery disease. Am J Med, 95, 23-8.

Chen, F., H. Kan, G. Hobbs \& M. S. Finkel (2009) p38 MAP kinase inhibitor reverses stress-induced myocardial dysfunction in vivo. J Appl Physiol, 106, 1132-41.

Chen, F., W. Lewis, J. M. Hollander, W. A. Baseler \& M. S. Finkel (2012) N-acetylcysteine Reverses Cardiac Myocyte Dysfunction in HIV-Tat Proteinopathy. J Appl Physiol.

Choy, K. H., O. Dean, M. Berk, A. I. Bush \& M. van den Buuse (2010) Effects of N-acetyl-cysteine treatment on glutathione depletion and a short-term spatial memory deficit in 2-cyclohexene-1one-treated rats. Eur J Pharmacol, 649, 224-8.

Chrousos, G. P. \& P. W. Gold (1992) The concepts of stress and stress system disorders. Overview of physical and behavioral homeostasis. JAMA, 267, 1244-52.

Clarke, A. S. \& M. L. Schneider (1993) Prenatal stress has long-term effects on behavioral responses to stress in juvenile rhesus monkeys. Dev Psychobiol, 26, 293-304.

Coplan, J. D., M. W. Andrews, L. A. Rosenblum, M. J. Owens, S. Friedman, J. M. Gorman \& C. B. Nemeroff (1996) Persistent elevations of cerebrospinal fluid concentrations of corticotropin-releasing factor in adult nonhuman primates exposed to early-life stressors: implications for the pathophysiology of mood and anxiety disorders. Proc Natl Acad Sci U S A, 93, 1619-23.

Cratty, M. S., H. E. Ward, E. A. Johnson, A. J. Azzaro \& D. L. Birkle (1995) Prenatal stress increases corticotropin-releasing factor (CRF) content and release in rat amygdala minces. Brain Res, 675, 297-302.

Dash, R., V. Kadambi, A. G. Schmidt, N. M. Tepe, D. Biniakiewicz, M. J. Gerst, A. M. Canning, W. T. Abraham, B. D. Hoit, S. B. Liggett, J. N. Lorenz, G. W. Dorn \& E. G. Kranias (2001) Interactions between phospholamban and beta-adrenergic drive may lead to cardiomyopathy and early mortality. Circulation, 103, 889-96.

Dash, R., A. G. Schmidt, A. Pathak, M. J. Gerst, D. Biniakiewicz, V. J. Kadambi, B. D. Hoit, W. T. Abraham \& E. G. Kranias (2003) Differential regulation of p38 mitogen-activated protein kinase mediates gender-dependent catecholamine-induced hypertrophy. Cardiovasc Res, 57, 704-14.

De Rosa, S. C., M. D. Zaretsky, J. G. Dubs, M. Roederer, M. Anderson, A. Green, D. Mitra, N. Watanabe, H. Nakamura, I. Tjioe, S. C. Deresinski, W. A. Moore, S. W. Ela, D. Parks \& L. A. Herzenberg (2000) $\mathrm{N}$-acetylcysteine replenishes glutathione in HIV infection. Eur J Clin Invest, 30, 915-29.

Deminière, J. M., P. V. Piazza, G. Guegan, N. Abrous, S. Maccari, M. Le Moal \& H. Simon (1992) Increased locomotor response to novelty and propensity to intravenous amphetamine self-administration in adult offspring of stressed mothers. Brain Res, 586, 135-9.

DiMatteo, M. R., P. J. Giordani, H. S. Lepper \& T. W. Croghan (2002) Patient adherence and medical treatment outcomes: a meta-analysis. Med Care, 40, 794-811.

Estanislau, C. \& S. Morato (2005) Prenatal stress produces more behavioral alterations than maternal separation in the elevated plus-maze and in the elevated T-maze. Behav Brain Res, 163, 70-7.

Fan, J. M., X. Q. Chen, H. Jin \& J. Z. Du (2009) Gestational hypoxia alone or combined with restraint sensitizes the hypothalamic-pituitary-adrenal axis and induces anxiety-like behavior in adult male rat offspring. Neuroscience, 159, 1363-73. 
Filomeni, G., G. Rotilio \& M. R. Ciriolo (2003) Glutathione disulfide induces apoptosis in U937 cells by a redox-mediated p38 MAP kinase pathway. FASEB J, 17, 64-6.

Finkel, M. S., L. Shen, C. V. Oddis, R. C. Romeo \& G. Salama (1993) Positive inotropic effect of acetylcysteine in cardiomyopathic Syrian hamsters. J Cardiovasc Pharmacol, 21, 29-34.

Frasure-Smith, N., F. Lespérance \& M. Talajic (1993) Depression following myocardial infarction. Impact on 6-month survival. JAMA, 270, 1819-25.

--- (1995) Depression and 18-month prognosis after myocardial infarction. Circulation, 91, 999-1005.

Fride, E., Y. Dan, J. Feldon, G. Halevy \& M. Weinstock (1986) Effects of prenatal stress on vulnerability to stress in prepubertal and adult rats. Physiol Behav, 37, 681-7.

Fride, E. \& M. Weinstock (1984) The effects of prenatal exposure to predictable or unpredictable stress on early development in the rat. Dev Psychobiol, 17, 651-60.

--- (1988) Prenatal stress increases anxiety related behavior and alters cerebral lateralization of dopamine activity. Life Sci, 42, 1059-65.

Frye, C. A. \& Z. A. Orecki (2002) Prenatal stress produces deficits in socio-sexual behavior of cycling, but not hormone-primed, Long-Evans rats. Pharmacol Biochem Behav, 73, 53-60.

Gaburjakova, J. \& M. Gaburjakova (2006) Comparison of the effects exerted by luminal Ca2+ on the sensitivity of the cardiac ryanodine receptor to caffeine and cytosolic Ca2+. J Membr Biol, 212, 17-28.

Glassman, A. H., J. E. Helzer, L. S. Covey, L. B. Cottler, F. Stetner, J. E. Tipp \& J. Johnson (1990) Smoking, smoking cessation, and major depression. JAMA, 264, 1546-9.

Glassman, A. H., C. M. O'Connor, R. M. Califf, K. Swedberg, P. Schwartz, J. T. Bigger, K. R. Krishnan, L. T. van Zyl, J. R. Swenson, M. S. Finkel, C. Landau, P. A. Shapiro, C. J. Pepine, J. Mardekian, W. M. Harrison, D. Barton, M. Mclvor \& S. A. H. A. R. T. S. Group (2002) Sertraline treatment of major depression in patients with acute MI or unstable angina. JAMA, 288, 701-9.

Goldhaber, J. I. \& M. S. Qayyum (2000) Oxygen free radicals and excitation-contraction coupling. Antioxid Redox Signal, 2, 55-64.

Gonzalez, D. R., A. V. Treuer, J. Castellanos, R. A. Dulce \& J. M. Hare (2010) Impaired S-nitrosylation of the ryanodine receptor caused by xanthine oxidase activity contributes to calcium leak in heart failure. J Biol Chem, 285, 28938-45.

Guck, T. P., G. N. Elsasser, M. G. Kavan \& E. J. Barone (2003) Depression and congestive heart failure. Congest Heart Fail, 9, 163-9.

Györke, S. \& C. Carnes (2008) Dysregulated sarcoplasmic reticulum calcium release: potential pharmacological target in cardiac disease. Pharmacol Ther, 119, 340-54.

Han, Y. H., Y. M. Yang, S. Z. Kim \& W. H. Park (2010) Attenuation of MG132-induced HeLa cell death by $\mathrm{N}$-acetyl cysteine via reducing reactive oxygen species and preventing glutathione depletion. Anticancer Res, 30, 2107-12.

Harada, D., M. Anraku, H. Fukuda, S. Naito, K. Harada, A. Suenaga \& M. Otagiri (2004) Kinetic studies of covalent binding between $\mathrm{N}$-acetyl-L-cysteine and human serum albumin through a mixeddisulfide using an N-methylpyridinium polymer-based column. Drug Metab Pharmacokinet, 19, 297-302.

Hariri, A. R., V. S. Mattay, A. Tessitore, B. Kolachana, F. Fera, D. Goldman, M. F. Egan \& D. R. Weinberger (2002) Serotonin transporter genetic variation and the response of the human amygdala. Science, 297, 400-3.

Hasenfuss, G. \& B. Pieske (2002) Calcium cycling in congestive heart failure. J Mol Cell Cardiol, 34, 95169.

Henry, C., M. Kabbaj, H. Simon, M. Le Moal \& S. Maccari (1994) Prenatal stress increases the hypothalamo-pituitary-adrenal axis response in young and adult rats. J Neuroendocrinol, 6, 3415. 
Hobai, I. A. \& B. O'Rourke (2001) Decreased sarcoplasmic reticulum calcium content is responsible for defective excitation-contraction coupling in canine heart failure. Circulation, 103, 1577-84.

Holdiness, M. R. (1991) Clinical pharmacokinetics of N-acetylcysteine. Clin Pharmacokinet, 20, 123-34.

Houser, S. R. \& K. B. Margulies (2003) Is depressed myocyte contractility centrally involved in heart failure? Circ Res, 92, 350-8.

Inesi, G., A. M. Prasad \& R. Pilankatta (2008) The Ca2+ ATPase of cardiac sarcoplasmic reticulum: Physiological role and relevance to diseases. Biochem Biophys Res Commun, 369, 182-7.

Jiang, W., M. Babyak, D. S. Krantz, R. A. Waugh, R. E. Coleman, M. M. Hanson, D. J. Frid, S. McNulty, J. J. Morris, C. M. O'Connor \& J. A. Blumenthal (1996) Mental stress--induced myocardial ischemia and cardiac events. JAMA, 275, 1651-6.

Johnson, G. L. \& R. Lapadat (2002) Mitogen-activated protein kinase pathways mediated by ERK, JNK, and p38 protein kinases. Science, 298, 1911-2.

Jones, D. P. (2008) Radical-free biology of oxidative stress. Am J Physiol Cell Physiol, 295, C849-68.

Joyeux, M., A. Boumendjel, R. Carroll, C. Ribuot, D. Godin-Ribuot \& D. M. Yellon (2000) SB 203580, a mitogen-activated protein kinase inhibitor, abolishes resistance to myocardial infarction induced by heat stress. Cardiovasc Drugs Ther, 14, 337-43.

Kamper, E. F., A. Chatzigeorgiou, O. Tsimpoukidi, M. Kamper, C. Dalla, P. M. Pitychoutis \& Z. Papadopoulou-Daifoti (2009) Sex differences in oxidant/antioxidant balance under a chronic mild stress regime. Physiol Behav, 98, 215-22.

Kan, H., D. Birkle, A. C. Jain, C. Failinger, S. Xie \& M. S. Finkel (2005) p38 MAP kinase inhibitor reverses stress-induced cardiac myocyte dysfunction. J Appl Physiol, 98, 77-82.

Kan, H. \& M. S. Finkel (2003) Inflammatory mediators and reversible myocardial dysfunction. J Cell Physiol, 195, 1-11.

Kan, H., Z. Xie \& M. S. Finkel (2004) p38 MAP kinase-mediated negative inotropic effect of HIV gp120 on cardiac myocytes. Am J Physiol Cell Physiol, 286, C1-7.

Kaye, D. M., S. D. Wiviott \& R. A. Kelly (1999) Activation of nitric oxide synthase (NOS3) by mechanical activity alters contractile activity in a Ca2+-independent manner in cardiac myocytes: role of troponin I phosphorylation. Biochem Biophys Res Commun, 256, 398-403.

Kelly, G. S. (1998) Clinical applications of N-acetylcysteine. Altern Med Rev, 3, 114-27.

Kerksick, C. \& D. Willoughby (2005) The antioxidant role of glutathione and N-acetyl-cysteine supplements and exercise-induced oxidative stress. J Int Soc Sports Nutr, 2, 38-44.

Khanna, D., H. Kan, C. Failinger, A. C. Jain \& M. S. Finkel (2006) Emotional stress and reversible myocardial dysfunction. Cardiovasc Toxicol, 6, 183-98.

Koenig, J. I., G. I. Elmer, P. D. Shepard, P. R. Lee, C. Mayo, B. Joy, E. Hercher \& D. L. Brady (2005) Prenatal exposure to a repeated variable stress paradigm elicits behavioral and neuroendocrinological changes in the adult offspring: potential relevance to schizophrenia. Behav Brain Res, 156, 25161.

Krantz, D. S., K. F. Helmers, C. N. Bairey, L. E. Nebel, S. M. Hedges \& A. Rozanski (1991) Cardiovascular reactivity and mental stress-induced myocardial ischemia in patients with coronary artery disease. Psychosom Med, 53, 1-12.

Kurisu, S., H. Sato, T. Kawagoe, M. Ishihara, Y. Shimatani, K. Nishioka, Y. Kono, T. Umemura \& S. Nakamura (2002) Tako-tsubo-like left ventricular dysfunction with ST-segment elevation: a novel cardiac syndrome mimicking acute myocardial infarction. Am Heart J, 143, 448-55.

Kuroda, J., T. Ago, S. Matsushima, P. Zhai, M. D. Schneider \& J. Sadoshima (2010) NADPH oxidase 4 (Nox4) is a major source of oxidative stress in the failing heart. Proc Natl Acad Sci U S A, 107, 15565-70.

Kushnir, A. \& A. R. Marks (2010) The ryanodine receptor in cardiac physiology and disease. Adv Pharmacol, 59, 1-30. 
Kuster, G. M., S. Lancel, J. Zhang, C. Communal, M. P. Trucillo, C. C. Lim, O. Pfister, E. O. Weinberg, R. A. Cohen, R. Liao, D. A. Siwik \& W. S. Colucci (2010) Redox-mediated reciprocal regulation of SERCA and $\mathrm{Na}+\mathrm{Ca} 2+$ exchanger contributes to sarcoplasmic reticulum $\mathrm{Ca} 2+$ depletion in cardiac myocytes. Free Radic Biol Med, 48, 1182-7.

Lancel, S., F. Qin, S. L. Lennon, J. Zhang, X. Tong, M. J. Mazzini, Y. J. Kang, D. A. Siwik, R. A. Cohen \& W. S. Colucci (2010) Oxidative posttranslational modifications mediate decreased SERCA activity and myocyte dysfunction in Galphaq-overexpressing mice. Circ Res, 107, 228-32.

Lauzon, C., C. A. Beck, T. Huynh, D. Dion, N. Racine, S. Carignan, J. G. Diodati, F. Charbonneau, R. Dupuis \& L. Pilote (2003) Depression and prognosis following hospital admission because of acute myocardial infarction. CMAJ, 168, 547-52.

Lespérance, F., N. Frasure-Smith, M. Talajic \& M. G. Bourassa (2002) Five-year risk of cardiac mortality in relation to initial severity and one-year changes in depression symptoms after myocardial infarction. Circulation, 105, 1049-53.

Li, S., M. Q. Zheng \& G. J. Rozanski (2009) Glutathione homeostasis in ventricular myocytes from rat hearts with chronic myocardial infarction. Exp Physiol, 94, 815-24.

Liang, Q. \& J. D. Molkentin (2003) Redefining the roles of p38 and JNK signaling in cardiac hypertrophy: dichotomy between cultured myocytes and animal models. J Mol Cell Cardiol, 35, 1385-94.

Liao, P., D. Georgakopoulos, A. Kovacs, M. Zheng, D. Lerner, H. Pu, J. Saffitz, K. Chien, R. P. Xiao, D. A. Kass \& Y. Wang (2001) The in vivo role of p38 MAP kinases in cardiac remodeling and restrictive cardiomyopathy. Proc Natl Acad Sci U S A, 98, 12283-8.

Liao, P., S. Q. Wang, S. Wang, M. Zheng, S. J. Zhang, H. Cheng, Y. Wang \& R. P. Xiao (2002) p38 Mitogenactivated protein kinase mediates a negative inotropic effect in cardiac myocytes. Circ Res, 90, 190-6.

Liu, J., X. Wang, M. K. Shigenaga, H. C. Yeo, A. Mori \& B. N. Ames (1996) Immobilization stress causes oxidative damage to lipid, protein, and DNA in the brain of rats. FASEB J, 10, 1532-8.

Liu, R., I. Y. Liu, X. Bi, R. F. Thompson, S. R. Doctrow, B. Malfroy \& M. Baudry (2003) Reversal of agerelated learning deficits and brain oxidative stress in mice with superoxide dismutase/catalase mimetics. Proc Natl Acad Sci U S A, 100, 8526-31.

Luo, J., Y. T. Xuan, Y. Gu \& S. D. Prabhu (2006) Prolonged oxidative stress inverts the cardiac forcefrequency relation: role of altered calcium handling and myofilament calcium responsiveness. $J$ Mol Cell Cardiol, 40, 64-75.

Maccari, S. \& S. Morley-Fletcher (2007) Effects of prenatal restraint stress on the hypothalamuspituitary-adrenal axis and related behavioural and neurobiological alterations. Psychoneuroendocrinology, 32 Suppl 1, S10-5.

Marx, S. O., J. Gaburjakova, M. Gaburjakova, C. Henrikson, K. Ondrias \& A. R. Marks (2001) Coupled gating between cardiac calcium release channels (ryanodine receptors). Circ Res, 88, 1151-8.

Marx, S. O., S. Reiken, Y. Hisamatsu, T. Jayaraman, D. Burkhoff, N. Rosemblit \& A. R. Marks (2000) PKA phosphorylation dissociates FKBP12.6 from the calcium release channel (ryanodine receptor): defective regulation in failing hearts. Cell, 101, 365-76.

Masood, A., A. Nadeem, S. J. Mustafa \& J. M. O'Donnell (2008) Reversal of oxidative stress-induced anxiety by inhibition of phosphodiesterase-2 in mice. J Pharmacol Exp Ther, 326, 369-79.

Mastorci, F., M. Vicentini, O. Viltart, M. Manghi, G. Graiani, F. Quaini, P. Meerlo, E. Nalivaiko, S. Maccari \& A. Sgoifo (2009) Long-term effects of prenatal stress: changes in adult cardiovascular regulation and sensitivity to stress. Neurosci Biobehav Rev, 33, 191-203.

Nadeem, A., A. Masood, N. Masood, R. A. Gilani \& Z. A. Shah (2006) Immobilization stress causes extracellular oxidant-antioxidant imbalance in rats: restoration by L-NAME and vitamin E. Eur Neuropsychopharmacol, 16, 260-7. 
Nakamura, H., H. Masutani \& J. Yodoi (2002) Redox imbalance and its control in HIV infection. Antioxid Redox Signal, 4, 455-64.

Nemeroff, C. B., E. Widerlöv, G. Bissette, H. Walléus, I. Karlsson, K. Eklund, C. D. Kilts, P. T. Loosen \& W. Vale (1984) Elevated concentrations of CSF corticotropin-releasing factor-like immunoreactivity in depressed patients. Science, 226, 1342-4.

Neri, M., D. Cerretani, A. I. Fiaschi, P. F. Laghi, P. E. Lazzerini, A. B. Maffione, L. Micheli, G. Bruni, C. Nencini, G. Giorgi, S. D'Errico, C. Fiore, C. Pomara, I. Riezzo, E. Turillazzi \& V. Fineschi (2007) Correlation between cardiac oxidative stress and myocardial pathology due to acute and chronic norepinephrine administration in rats. J Cell Mol Med, 11, 156-70.

Nilsson, C., B. M. Larsson, E. Jennische, E. Eriksson, P. Björntorp, D. A. York \& A. Holmäng (2001) Maternal endotoxemia results in obesity and insulin resistance in adult male offspring. Endocrinology, 142, 2622-30.

Ohkawa, T., W. Rohde, S. Takeshita, G. Dörner, K. Arai \& S. Okinaga (1991) Effect of an acute maternal stress on the fetal hypothalamo-pituitary-adrenal system in late gestational life of the rat. Exp Clin Endocrinol, 98, 123-9.

Osadchii, O. E. (2007) Cardiac hypertrophy induced by sustained beta-adrenoreceptor activation: pathophysiological aspects. Heart Fail Rev, 12, 66-86.

Pignay-Demaria, V., F. Lespérance, R. G. Demaria, N. Frasure-Smith \& L. P. Perrault (2003) Depression and anxiety and outcomes of coronary artery bypass surgery. Ann Thorac Surg, 75, 314-21.

Prabhu, S. D. \& G. Salama (1990) Reactive disulfide compounds induce Ca2+ release from cardiac sarcoplasmic reticulum. Arch Biochem Biophys, 282, 275-83.

Prosser, B. L., C. W. Ward \& W. J. Lederer (2010) Subcellular Ca2+ signaling in the heart: the role of ryanodine receptor sensitivity. J Gen Physiol, 136, 135-42.

Rapacciuolo, A., G. Esposito, K. Caron, L. Mao, S. A. Thomas \& H. A. Rockman (2001) Important role of endogenous norepinephrine and epinephrine in the development of in vivo pressure-overload cardiac hypertrophy. J Am Coll Cardiol, 38, 876-82.

Reeves, J. P., C. A. Bailey \& C. C. Hale (1986) Redox modification of sodium-calcium exchange activity in cardiac sarcolemmal vesicles. J Biol Chem, 261, 4948-55.

Richard, C. (2011) Stress-related cardiomyopathies. Ann Intensive Care, 1, 39.

Richardson, H. N., E. P. Zorrilla, C. D. Mandyam \& C. L. Rivier (2006) Exposure to repetitive versus varied stress during prenatal development generates two distinct anxiogenic and neuroendocrine profiles in adulthood. Endocrinology, 147, 2506-17.

Roederer, M., S. W. Ela, F. J. Staal \& L. A. Herzenberg (1992) N-acetylcysteine: a new approach to antiHIV therapy. AIDS Res Hum Retroviruses, 8, 209-17.

Saitoh, S., T. Kiyooka, P. Rocic, P. A. Rogers, C. Zhang, A. Swafford, G. M. Dick, C. Viswanathan, Y. Park \& W. M. Chilian (2007) Redox-dependent coronary metabolic dilation. Am J Physiol Heart Circ Physiol, 293, H3720-5.

Sakamoto, A., K. Ono, M. Abe, G. Jasmin, T. Eki, Y. Murakami, T. Masaki, T. Toyo-oka \& F. Hanaoka (1997) Both hypertrophic and dilated cardiomyopathies are caused by mutation of the same gene, delta-sarcoglycan, in hamster: an animal model of disrupted dystrophin-associated glycoprotein complex. Proc Natl Acad Sci U S A, 94, 13873-8.

Salama, G., J. J. Abramson \& G. K. Pike (1992) Sulphydryl reagents trigger Ca2+ release from the sarcoplasmic reticulum of skinned rabbit psoas fibres. J Physiol, 454, 389-420.

Salm, A. K., M. Pavelko, E. M. Krouse, W. Webster, M. Kraszpulski \& D. L. Birkle (2004) Lateral amygdaloid nucleus expansion in adult rats is associated with exposure to prenatal stress. Brain Res Dev Brain Res, 148, 159-67.

Satoh, N., T. M. Suter, R. Liao \& W. S. Colucci (2000) Chronic alpha-adrenergic receptor stimulation modulates the contractile phenotype of cardiac myocytes in vitro. Circulation, 102, 2249-54. 
Sawyer, D. B., D. A. Siwik, L. Xiao, D. R. Pimentel, K. Singh \& W. S. Colucci (2002) Role of oxidative stress in myocardial hypertrophy and failure. J Mol Cell Cardiol, 34, 379-88.

Scherer, N. M. \& D. W. Deamer (1986) Oxidative stress impairs the function of sarcoplasmic reticulum by oxidation of sulfhydryl groups in the Ca2+-ATPase. Arch Biochem Biophys, 246, 589-601.

Schneider, M. L. (1992) Prenatal stress exposure alters postnatal behavioral expression under conditions of novelty challenge in rhesus monkey infants. Dev Psychobiol, 25, 529-40.

Shan, J., M. J. Betzenhauser, A. Kushnir, S. Reiken, A. C. Meli, A. Wronska, M. Dura, B. X. Chen \& A. R. Marks (2010a) Role of chronic ryanodine receptor phosphorylation in heart failure and $\beta$ adrenergic receptor blockade in mice. J Clin Invest, 120, 4375-87.

Shan, J., A. Kushnir, M. J. Betzenhauser, S. Reiken, J. Li, S. E. Lehnart, N. Lindegger, M. Mongillo, P. J. Mohler \& A. R. Marks (2010b) Phosphorylation of the ryanodine receptor mediates the cardiac fight or flight response in mice. J Clin Invest, 120, 4388-98.

Sharkey, S. W., J. R. Lesser, A. G. Zenovich, M. S. Maron, J. Lindberg, T. F. Longe \& B. J. Maron (2005) Acute and reversible cardiomyopathy provoked by stress in women from the United States. Circulation, 111, 472-9.

Shi, Y. \& M. Gaestel (2002) In the cellular garden of forking paths: how p38 MAPKs signal for downstream assistance. Biol Chem, 383, 1519-36.

Song, L., J. Zheng, H. Li, N. Jia, Z. Suo, Q. Cai, Z. Bai, D. Cheng \& Z. Zhu (2009) Prenatal stress causes oxidative damage to mitochondrial DNA in hippocampus of offspring rats. Neurochem Res, 34, 739-45.

Sutor, B., T. A. Rummans, S. G. Jowsey, L. E. Krahn, M. J. Martin, M. K. O'Connor, K. L. Philbrick \& J. W. Richardson (1998) Major depression in medically ill patients. Mayo Clin Proc, 73, 329-37.

Tafet, G. E., V. P. Idoyaga-Vargas, D. P. Abulafia, J. M. Calandria, S. S. Roffman, A. Chiovetta \& M. Shinitzky (2001) Correlation between cortisol level and serotonin uptake in patients with chronic stress and depression. Cogn Affect Behav Neurosci, 1, 388-93.

Takahashi, L. K., E. W. Baker \& N. H. Kalin (1990) Ontogeny of behavioral and hormonal responses to stress in prenatally stressed male rat pups. Physiol Behav, 47, 357-64.

Takahashi, L. K. \& N. H. Kalin (1991) Early developmental and temporal characteristics of stress-induced secretion of pituitary-adrenal hormones in prenatally stressed rat pups. Brain Res, 558, 75-8.

Takahashi, L. K., N. H. Kalin, C. M. Barksdale, J. A. Vanden Burgt \& M. S. Brownfield (1988) Stressor controllability during pregnancy influences pituitary-adrenal hormone concentrations and analgesic responsiveness in offspring. Physiol Behav, 42, 323-9.

Takahashi, L. K., J. G. Turner \& N. H. Kalin (1992) Prenatal stress alters brain catecholaminergic activity and potentiates stress-induced behavior in adult rats. Brain Res, 574, 131-7.

--- (1998) Prolonged stress-induced elevation in plasma corticosterone during pregnancy in the rat: implications for prenatal stress studies. Psychoneuroendocrinology, 23, 571-81.

Takeishi, Y., G. Chu, D. M. Kirkpatrick, Z. Li, H. Wakasaki, E. G. Kranias, G. L. King \& R. A. Walsh (1998) In vivo phosphorylation of cardiac troponin I by protein kinase Cbeta2 decreases cardiomyocyte calcium responsiveness and contractility in transgenic mouse hearts. J Clin Invest, 102, 72-8.

Tang, W. H., G. M. Kravtsov, M. Sauert, X. Y. Tong, X. Y. Hou, T. M. Wong, S. K. Chung \& S. S. Man Chung (2010) Polyol pathway impairs the function of SERCA and RyR in ischemic-reperfused rat hearts by increasing oxidative modifications of these proteins. J Mol Cell Cardiol, 49, 58-69.

Tazumi, T., E. Hori, T. Uwano, K. Umeno, K. Tanebe, E. Tabuchi, T. Ono \& H. Nishijo (2005) Effects of prenatal maternal stress by repeated cold environment on behavioral and emotional development in the rat offspring. Behav Brain Res, 162, 153-60.

Terentyev, D., I. Györke, A. E. Belevych, R. Terentyeva, A. Sridhar, Y. Nishijima, E. C. de Blanco, S. Khanna, C. K. Sen, A. J. Cardounel, C. A. Carnes \& S. Györke (2008) Redox modification of 
ryanodine receptors contributes to sarcoplasmic reticulum $\mathrm{Ca} 2+$ leak in chronic heart failure. Circ Res, 103, 1466-72.

Trimm, J. L., G. Salama \& J. J. Abramson (1986) Sulfhydryl oxidation induces rapid calcium release from sarcoplasmic reticulum vesicles. J Biol Chem, 261, 16092-8.

Vallée, M., W. Mayo, F. Dellu, M. Le Moal, H. Simon \& S. Maccari (1997) Prenatal stress induces high anxiety and postnatal handling induces low anxiety in adult offspring: correlation with stressinduced corticosterone secretion. J Neurosci, 17, 2626-36.

Veal, E. A., V. J. Findlay, A. M. Day, S. M. Bozonet, J. M. Evans, J. Quinn \& B. A. Morgan (2004) A 2-Cys peroxiredoxin regulates peroxide-induced oxidation and activation of a stress-activated MAP kinase. Mol Cell, 15, 129-39.

Veith, R. C., N. Lewis, O. A. Linares, R. F. Barnes, M. A. Raskind, E. C. Villacres, M. M. Murburg, E. A. Ashleigh, S. Castillo \& E. R. Peskind (1994) Sympathetic nervous system activity in major depression. Basal and desipramine-induced alterations in plasma norepinephrine kinetics. Arch Gen Psychiatry, 51, 411-22.

Wakshlak, A. \& M. Weinstock (1990) Neonatal handling reverses behavioral abnormalities induced in rats by prenatal stress. Physiol Behav, 48, 289-92.

Wang, Y., S. Huang, V. P. Sah, J. Ross, J. H. Brown, J. Han \& K. R. Chien (1998) Cardiac muscle cell hypertrophy and apoptosis induced by distinct members of the p38 mitogen-activated protein kinase family. J Biol Chem, 273, 2161-8.

Ward, H. E., E. A. Johnson, A. K. Salm \& D. L. Birkle (2000) Effects of prenatal stress on defensive withdrawal behavior and corticotropin releasing factor systems in rat brain. Physiol Behav, 70, 359-66.

Ward, I. L. \& J. Weisz (1984) Differential effects of maternal stress on circulating levels of corticosterone, progesterone, and testosterone in male and female rat fetuses and their mothers. Endocrinology, 114, 1635-44.

Weinstock, M. (1997) Does prenatal stress impair coping and regulation of hypothalamic-pituitaryadrenal axis? Neurosci Biobehav Rev, 21, 1-10.

Weinstock, M., E. Fride \& R. Hertzberg (1988) Prenatal stress effects on functional development of the offspring. Prog Brain Res, 73, 319-31.

Weinstock, M., T. Poltyrev, D. Schorer-Apelbaum, D. Men \& R. McCarty (1998) Effect of prenatal stress on plasma corticosterone and catecholamines in response to footshock in rats. Physiol Behav, 64, 439-44.

Wenzel, S., C. Müller, H. M. Piper \& K. D. Schlüter (2005) p38 MAP-kinase in cultured adult rat ventricular cardiomyocytes: expression and involvement in hypertrophic signalling. Eur J Heart Fail, 7, 453-60.

Williams, I. A. \& D. G. Allen (2007) The role of reactive oxygen species in the hearts of dystrophindeficient mdx mice. Am J Physiol Heart Circ Physiol, 293, H1969-77.

Williams, M. T., H. N. Davis, A. E. McCrea, S. J. Long \& M. B. Hennessy (1999) Changes in the hormonal concentrations of pregnant rats and their fetuses following multiple exposures to a stressor during the third trimester. Neurotoxicol Teratol, 21, 403-14.

Wittstein, I. S., D. R. Thiemann, J. A. Lima, K. L. Baughman, S. P. Schulman, G. Gerstenblith, K. C. Wu, J. J. Rade, T. J. Bivalacqua \& H. C. Champion (2005) Neurohumoral features of myocardial stunning due to sudden emotional stress. N Engl J Med, 352, 539-48.

Xu, Q., A. Dalic, L. Fang, H. Kiriazis, R. H. Ritchie, K. Sim, X. M. Gao, G. Drummond, M. Sarwar, Y. Y. Zhang, A. M. Dart \& X. J. Du (2011) Myocardial oxidative stress contributes to transgenic $\beta_{2^{-}}$ adrenoceptor activation-induced cardiomyopathy and heart failure. Br J Pharmacol, 162, 1012 28. 
Zafarullah, M., W. Q. Li, J. Sylvester \& M. Ahmad (2003) Molecular mechanisms of N-acetylcysteine actions. Cell Mol Life Sci, 60, 6-20.

Zalk, R., S. E. Lehnart \& A. R. Marks (2007) Modulation of the ryanodine receptor and intracellular calcium. Annu Rev Biochem, 76, 367-85.

Zechner, D., R. Craig, D. S. Hanford, P. M. McDonough, R. A. Sabbadini \& C. C. Glembotski (1998) MKK6 activates myocardial cell NF-kappaB and inhibits apoptosis in a p38 mitogen-activated protein kinase-dependent manner. J Biol Chem, 273, 8232-9.

Zechner, D., D. J. Thuerauf, D. S. Hanford, P. M. McDonough \& C. C. Glembotski (1997) A role for the p38 mitogen-activated protein kinase pathway in myocardial cell growth, sarcomeric organization, and cardiac-specific gene expression. J Cell Biol, 139, 115-27.

Zheng, M., S. J. Zhang, W. Z. Zhu, B. Ziman, B. K. Kobilka \& R. P. Xiao (2000) beta 2-adrenergic receptorinduced $\mathrm{p} 38$ MAPK activation is mediated by protein kinase $A$ rather than by $\mathrm{Gi}$ or gbeta gamma in adult mouse cardiomyocytes. J Biol Chem, 275, 40635-40.

Zhu, Z., X. Li, W. Chen, Y. Zhao, H. Li, C. Qing, N. Jia, Z. Bai \& J. Liu (2004) Prenatal stress causes genderdependent neuronal loss and oxidative stress in rat hippocampus. J Neurosci Res, 78, 837-44.

Ziegelstein, R. C., J. A. Fauerbach, S. S. Stevens, J. Romanelli, D. P. Richter \& D. E. Bush (2000) Patients with depression are less likely to follow recommendations to reduce cardiac risk during recovery from a myocardial infarction. Arch Intern Med, 160, 1818-23.

Zima, A. V. \& L. A. Blatter (2006) Redox regulation of cardiac calcium channels and transporters. Cardiovasc Res, 71, 310-21.

Zima, A. V., E. Bovo, D. M. Bers \& L. A. Blatter (2010) $\mathrm{Ca}^{2}+$ spark-dependent and -independent sarcoplasmic reticulum $\mathrm{Ca}^{2}+$ leak in normal and failing rabbit ventricular myocytes. J Physiol, 588, 4743-57.

Zimmerberg, B. \& L. G. Blaskey (1998) Prenatal stress effects are partially ameliorated by prenatal administration of the neurosteroid allopregnanolone. Pharmacol Biochem Behav, 59, 819-27. 